Japan. J. Math.

Vol. 18, No. 2, 1992

\title{
On completely reducible transitive graded Lie algebras of finite depth
}

\author{
By Tomoaki Yatsui
}

(Received November 5, 1990)

(Revised September 11, 1991)

\section{Introduction}

Let $\mathfrak{g}=\bigoplus_{p \in \mathbf{Z}} \mathfrak{g}_{p}$ be a graded Lie algebra (GLA) over $F=\mathbf{R}$ or $\mathbf{C}$. $\mathfrak{g}=$ $\bigoplus_{p \in \mathbf{Z}} \mathfrak{g}_{p}$ is said to be of finite depth if $\operatorname{dim} \mathfrak{g}_{-}<\infty$, where $\mathfrak{g}_{-}=\bigoplus_{p<0} \mathfrak{g}_{p}$. Moreover $\mathfrak{g}=\bigoplus_{p \in \mathbf{Z}} \mathfrak{g}_{p}$ is called transitive if $\mathfrak{g}_{-}$is generated by $\mathfrak{g}_{-1}$ and, for $x \in \mathfrak{g}_{p}(p \geq 0)$, $\left[x, \mathfrak{g}_{-}\right]=0$ implies $x=0$, and completely reducible (resp. irreducible) if the $\mathfrak{g}_{0^{-}}$ module $\mathfrak{g}_{-1}$ is so. Also, in this paper, given a GLA $\mathfrak{g}=\bigoplus_{p \in \mathbf{Z}} \mathfrak{g}_{p}$, we denote by $\mathfrak{g}^{a}=\bigoplus_{p \in \mathbf{Z}} \mathfrak{g}_{p}^{a}$ the graded ideal $\bigoplus_{p \neq 0} \mathfrak{g}_{p}+[\mathfrak{g}, \mathfrak{g}]$ of $\mathfrak{g}$.

The present paper is the sequel to the previous paper [Yat88]. The first aim of this paper is to investigate the structure of (possibly infinite dimensional) completely reducible transitive GLAs (c.r.t.-GLAs) of finite depth. The infinite dimensional case is motivated by the classification of the infinite dimensional irreducible transitive GLAs of finite depth in [KN65] and [MT70] and also by the classification of bitransitive irreducible GLAs of finite growth in [Kac68]. Note that a transitive GLA of finite depth is of finite growth. Our second aim is to investigate the structure of nonsemisimple pseudo-product GLAs, which is motivated by the study of geometric symbols of differential equations (cf. [Tan70]).

To explain our main result, recall the classification of infinite dimensional irreducible transitive GLA of finite depth. In [KN65] and [MT70], it was proved that any complex infinite dimensional irreducible transitive GLA satisfying the condition " $\mathfrak{g}^{a}=\mathfrak{g}$ " is isomorphic to one of the simple Lie algebras of Cartan type (i.e., the general algebras $W(n)$, the special algebras $S(n)$, the Hamiltonian algebras $H(n)$, and the contact algebras $K(n))$ with the standard gradation or the GLAs $\mathfrak{d}(n)$. Here the GLAs $\mathfrak{d}(n)$ are the graded subalgebras of $K(n)$ (with respect to the standard gradation) consisting of all the infinitesimal strict contact transformations and they are infinite dimensional irreducible transitive GLAs of finite depth.

In this paper we first show the following main theorem (In 2.3, this theorem will be described in a more sophisticated form): Let $\mathfrak{g}=\bigoplus_{p \in \mathbf{Z}} \mathfrak{g}_{p}$ be a complex c.r.t.-GLA of finite depth. Then the graded ideal $\mathfrak{g}^{a}$ of $\mathfrak{g}$ is a direct sum of graded 
ideals $\mathfrak{g}^{(i)}(i=0, \ldots, k)$ with the following properties:

(1) $\mathfrak{g}^{(i)}(i=1, \ldots, k)$ is isomorphic to one of simple Lie algebras of Cartan type with the standard gradation;

(2) $\mathfrak{g}^{(0)}$ is a (not necessarily direct) sum of graded ideals $\mathfrak{h}^{(i)}(i=0, \ldots, l)$ such that $\mathfrak{h}^{(0)}$ is a finite dimensional c.r.t.-GLA and each $\mathfrak{h}^{(i)}(i=1, \ldots, l)$ is an infinite dimensional irreducible transitive GLA of finite depth isomorphic to $\mathfrak{d}\left(n_{i}\right)$ for some $n_{i}$;

(3) $\mathfrak{g}^{(0)}$ is isomorphic to the factor algebra of the direct product $\mathcal{L}:=\prod_{i=0}^{l} \mathfrak{h}^{(i)}$ by an ideal $\mathfrak{a}$ of $\mathcal{L}$ such that $\mathfrak{a} \subset \mathfrak{z}\left(\mathfrak{h}^{(0)}\right) \cap \mathcal{L}_{-2} \bigoplus \bigoplus_{i=1}^{l} \mathfrak{h}^{(i)} \cap \mathcal{L}_{-2}$ and $\mathfrak{a} \cap \mathfrak{h}^{(i)}=\{0\}$ $(i=0, \ldots, l)$, where $\mathfrak{z}\left(\mathfrak{h}^{(0)}\right)$ is the center of $\mathfrak{h}^{(0)}$.

We also obtain a similar result in the case when the ground field is the field of real numbers (Theorem 23). Thus the investigation of the structure of c.r.t.-GLAs of finite depth is reduced to that of finite dimensional c.r.t.-GLAs. In [Yat88], we proved that a finite dimensional c.r.t.-GLA is a semi-direct product of a reductive GLA $\mathfrak{l}=\bigoplus_{p \in \mathbf{Z}} \mathfrak{l}_{p}$ by a nilpotent GLA $\mathfrak{n}=\bigoplus_{p<0} \mathfrak{n}_{p}$. However, it seems to us that the isomorphism classes of finite dimensional c.r.t.-GLAs are very vast and the exhaustive classification is not possible. Among finite dimensional c.r.t.-GLAs, completely reducible pseudo-product GLAs are of great interest. Therefore we restrict our attention to completely reducible pseudo-product GLAs.

Let $\mathfrak{g}=\bigoplus_{p \in \mathbf{Z}} \mathfrak{g}_{p}$ be a transitive GLA of finite depth and $\mathfrak{e}^{1}$ and $\mathfrak{e}^{2}$ be two $\mathfrak{g}_{0}$-submodules of $\mathfrak{g}_{-1}$. Then the system $\mathfrak{G}=\left(\mathfrak{g},\left(\mathfrak{g}_{p}\right)_{p \in \mathbf{Z}}, \mathfrak{e}^{1}, \mathfrak{e}^{2}\right)$ is called a pseudoproduct GLA if the following conditions hold:

(1) $\mathfrak{g}_{-1}=\mathfrak{e}^{1} \bigoplus \mathfrak{e}^{2}$

(2) $\left[\mathfrak{e}^{i}, \mathfrak{e}^{i}\right]=\{0\} \quad(i=1,2)$.

In particular, if each $\mathfrak{e}^{i}$ is an irreducible $\mathfrak{g}_{0}$-module and $\mathfrak{g}_{-2} \neq\{0\}$, then $\mathfrak{G}$ is called a pseudo-product GLA of irreducible type.

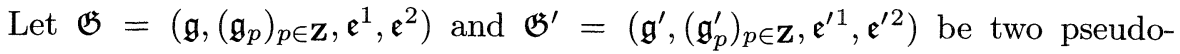
product GLAs. By an isomorphism of $\mathfrak{G}$ onto $\mathfrak{G}^{\prime}$, we mean an isomorphism $\phi$ of $\mathfrak{g}$ onto $\mathfrak{g}^{\prime}$ such that $\phi\left(\mathfrak{g}_{p}\right)=\mathfrak{g}_{p}^{\prime}$ for all $p \in \mathbf{Z}$ and $\phi\left(\mathfrak{e}^{i}\right)=\mathfrak{e}^{\prime \sigma(i)}$ for some permutation $\sigma$ of order 2 .

Before stating the second main result, we introduce two typical models of pseudo-product GLAs of irreducible type. Let $\mathfrak{l}=\mathfrak{l}_{-1} \oplus \mathfrak{l}_{0} \oplus \mathfrak{l}_{1}$ be a reductive GLA such that $\widehat{\mathfrak{l}}$ is simple and $\mathfrak{z}(\mathfrak{l}) \subset \mathfrak{l}_{0}$, where $\widehat{\mathfrak{l}}:=\mathfrak{l}_{-1} \bigoplus\left[\mathfrak{l}_{-1}, \mathfrak{l}_{1}\right] \bigoplus \mathfrak{l}_{1}$ and $\mathfrak{z}(\mathfrak{l})$ is the center of $\mathfrak{l}$. Further let $M=\bigoplus_{p=-1}^{-k} M_{p}(k \geq 2)$ be a faithful irreducible graded l-module such that $M_{-1} \neq\{0\}, N$ be an l-submodule of $\Lambda^{2}(M)$ and $\Phi$ be the projection of $\Lambda^{2}(M)$ onto $\Lambda^{2}(M) / N$. Considering $M$ as a commutative Lie algebra, we can define a semi-direct product $\mathfrak{g}=\mathfrak{l} \ltimes M$ of $\mathfrak{l}$ by $M$ and the gradation as follows: $\mathfrak{g}_{p}=M_{p}$ for $p \leq-2, \mathfrak{g}_{-1}=\mathfrak{l}_{-1} \oplus M_{-1}, \mathfrak{g}_{p}=\mathfrak{l}_{p}$ for $p \geq 0$; then the system $\mathfrak{G}=\left(\mathfrak{g},\left(\mathfrak{g}_{p}\right)_{p \in Z}, \mathfrak{l}_{-1}, M_{-1}\right)$ is a pseudo-product GLA of irreducible type, which is said to be of the irreducible type $(\mathfrak{l}, M)$. Next we assume that $k=2$, and the $\widehat{\mathfrak{l}}$-module $\Lambda^{2}(M) / N$ is non-zero and trivial. We set $R=R_{-1} \bigoplus R_{-2} \bigoplus R_{-3}$, 
$R_{-1}=M_{-1}, R_{-2}=M_{-2}, R_{-3}=\Lambda^{2}(M) / N$. If we define the bracket operation on $R$ by $\left[R, R_{-3}\right]=\{0\}$ and $[x, y]=\Phi(x \wedge y)(x, y \in M)$, then $R$ naturally becomes a nilpotent GLA such that $[R, R] \subset R_{-3}$. Further $R$ is a graded l-module and $\mathfrak{l}$ is considered as a subalgebra of the derivation algebra $\operatorname{Der}(R)$ of $R$. Thus we can define a semi-direct product $\mathfrak{g}=\mathfrak{l} \ltimes R$ of $\mathfrak{l}$ by $R$ and its gradation as follows: $\mathfrak{g}_{p}=R_{p}$ for $p \leq-2, \mathfrak{g}_{-1}=\mathfrak{l}_{-1} \bigoplus R_{-1}, \mathfrak{g}_{p}=\mathfrak{l}_{p}$ for $p \geq 0$. Then the system $\mathfrak{G}=\left(\mathfrak{g},\left(\mathfrak{g}_{p}\right)_{p \in \mathbf{Z}}, \mathfrak{l}_{-1}, R_{-1}\right)$ is a pseudo-product GLA of irreducible type, which is said to be of the irreducible type $(\mathfrak{l}, M, N)$ of order 2 . Also, in case $F=\mathbf{C}$, these GLAs are parametrized by a root system of $\mathfrak{l}$ and the highest weight of the $\mathfrak{l}$-module $M$.

In Section 4, we prove the following theorem (Theorem 32): Let $\mathfrak{G}=$ $\left(\mathfrak{g},\left(\mathfrak{g}_{p}\right)_{p \in \mathbf{Z}}, \mathfrak{e}^{1}, \mathfrak{e}^{2}\right)$ be a pseudo-product GLA of irreducible type satisfying the condition " $\mathfrak{d}_{-1}(\mathfrak{g}):=\left\{x \in \mathfrak{g}_{-1}:\left[x, \bigoplus_{p \leq-2} \mathfrak{g}_{p}\right]=\{0\}\right\} \neq\{0\}$ ". If $\mathfrak{g}_{1} \neq\{0\}$ and $\mathfrak{g}_{2}=\{0\}$, then $\mathfrak{G}$ is isomorphic to a pseudo-product GLA of the irreducible type $(\mathfrak{l}, M)$ or of the irreducible type $(\mathfrak{l}, M, N)$ of order 2 .

In [Yat88], we proved that if $\mathfrak{g}_{2} \neq\{0\}$, then the underlying Lie algebra $\mathfrak{g}$ of a pseudo-product GLA $\mathfrak{G}$ is simple. Thus we have accomplished the classification of pseudo-product GLAs of irreducible type such that $\mathfrak{d}_{-1}(\mathfrak{g}) \neq\{0\}$ and $\mathfrak{g}_{1} \neq\{0\}$.

We now give a brief description of the various sections. Following [Kac68] and [Kac70], we first give basic definitions on GLAs and local Lie algebras, and describe GLAs of Cartan type. Moreover we state the definition of truncated transitive GLAs of finite depth and pseudo-product GLAs. In Sections 2 and 3, we investigate the structure of c.r.t.-GLAs of finite depth over $F=\mathbf{C}$ or $\mathbf{R}$, which is the first main result. Our task in the sections is mainly divided into two parts. The first part is to determine the structure of the truncated subalgebra $\bigoplus_{p<1} \mathfrak{g}_{p}$ of $\mathfrak{g}$. To do this we apply the reasoning, due to V.G. Kac [Kac68], in the realization of GLAs, and use a criterion, due to N. Tanaka [Tan70], for transitive GLAs of finite depth to be finite dimensional. The second part is to determine the prolongation of $\bigoplus_{p \leq 1} \mathfrak{g}_{p}$. To do this we apply the results of [KN65] and [MT70]. In the rest of Section 4, we investigate the prolongation of $\mathfrak{g}_{-}$and the derivation algebra of the underlying Lie algebra $\mathfrak{g}$ for the pseudo-product GLAs $\mathfrak{G}$ of the irreducible type $(\mathfrak{l}, M)$ and of the irreducible type $(\mathfrak{l}, M, N)$ of order 2 . Moreover we investigate the automorphism group of the underlying Lie algebra $\mathfrak{g}$ in case $\mathfrak{G}$ is of type $(\mathfrak{l}, M)$.

\section{Notation and conventions}

(1) Let $\mathfrak{g}$ be a Lie algebra, $\mathfrak{a}$ be a subalgebra of $\mathfrak{g}$ and $M$ be a $\mathfrak{g}$-module. $\mathfrak{z}(\mathfrak{g})$ denotes the center of $\mathfrak{g}, \mathfrak{n}_{\mathfrak{g}}(\mathfrak{a})$ denotes the normalizer of $\mathfrak{a}$ in $\mathfrak{g}, \operatorname{Der}(\mathfrak{g})$ denotes the derivation algebra of $\mathfrak{g}$ and $\mathcal{L}_{\mathfrak{g}}(M)$ denotes the Lie algebra of all the homomorphisms of the $\mathfrak{g}$-module $M$. Let $\mathfrak{g}$ be a complex Lie algebra. We write $\mathfrak{g}_{\mathbf{R}}$ when we regard $\mathfrak{g}$ as a real Lie algebra. 
(2) A graded vector space $V$ is called a vector space with a $\mathbf{Z}$-gradation, i.e., a vector space equipped with a decomposition $V=\bigoplus_{p \in \mathbf{Z}} V_{p}$. In this paper, we will always assume that each homogeneous component $V_{p}$ is finite dimensional. If we write $V=\bigoplus_{p=s}^{t} V_{p}$, then it is understood that $V_{p}=0$ for $p<s$ or $p>t$. Given a graded vector space $V=\bigoplus_{p \in \mathbf{Z}} V_{p}$, we denote by $V_{-}$(resp. $V_{+}$) the graded subspace $\bigoplus_{p<0} V_{p}$ (resp. $\bigoplus_{p>0} V_{p}$ ) of $V$. For $k \in \mathbf{Z}$, we set $\operatorname{End}_{k}(V)=\{\varphi \in$ $\operatorname{End}(V): \varphi\left(V_{p}\right) \subset V_{p+k}$ for all $\left.p\right\}$.

\section{§1. Preliminaries}

In this section, the ground field $F$ is the field $\mathbf{C}$ of complex numbers or the field $\mathbf{R}$ of real numbers.

1.1. Graded Lie algebras.

A graded Lie algebra (GLA) $\mathfrak{g}=\bigoplus_{p \in \mathbf{Z}} \mathfrak{g}_{p}$ is a graded vector space endowed with a Lie algebra structure such that $\left[\mathfrak{g}_{p}, \mathfrak{g}_{q}\right] \subset \mathfrak{g}_{p+q}$. A subalgebra (resp. an ideal) $\mathfrak{s}$ of $\mathfrak{g}$ is called a graded subalgebra (resp. ideal) if $\mathfrak{s}=\bigoplus_{p \in \mathbf{Z}} \mathfrak{s} \cap \mathfrak{g}_{p}$.

Let $\mathfrak{g}=\bigoplus_{p \in \mathbf{Z}} \mathfrak{g}_{p}$ and $\mathfrak{g}^{\prime}=\bigoplus_{p \in \mathbf{Z}} \mathfrak{g}_{p}^{\prime}$ be two GLAs. Then a homomorphism $\varphi$ of $\mathfrak{g}$ into $\mathfrak{g}^{\prime}$, by definition, preserves the gradation in the sense that $\varphi\left(\mathfrak{g}_{p}\right) \subset$ $\mathfrak{g}_{p}^{\prime}$. Similarly isomorphisms, monomorphisms and epimorphisms are defined. Let $\mathfrak{g}=\bigoplus_{p \in \mathbf{Z}} \mathfrak{g}_{p}$ be a GLA. If the negative part $\mathfrak{g}_{-}$of $\mathfrak{g}$ is finite dimensional, then $\mathfrak{g}=\bigoplus_{p \in \mathbf{Z}} \mathfrak{g}_{p}$ is said to be of finite depth. Otherwise $\mathfrak{g}$ is said to be of infinite depth. In particular, if $\mathfrak{g}_{-\mu} \neq\{0\}$ and $\mathfrak{g}_{p}=\{0\}$ for $p<-\mu$, then $\mathfrak{g}$ is said to be of depth $\mu$.

In this paper, we call a GLA $\mathfrak{g}=\bigoplus_{p \in \mathbf{Z}} \mathfrak{g}_{p}$ transitive if the following conditions hold:

(1) $\mathfrak{g}_{-}$is generated by $\mathfrak{g}_{-1}$;

(2) For $x \in \mathfrak{g}_{p}(p \geq 0),\left[x, \mathfrak{g}_{-}\right]=\{0\}$ implies $x=0$.

(In general, the definition of transitivity does not contain (1).) We abbreviate transitive GLA to TGLA. Note that a TGLA $\mathfrak{g}=\bigoplus_{p \in \mathbf{Z}} \mathfrak{g}_{p}$ of finite depth is the so-called fundamental GLA (FGLA) in case $\mathfrak{g}_{p}=\{0\}$ for $p \geq 0$ (see [Tan70]). In this paper given a TGLA $\mathfrak{g}=\bigoplus_{p \in \mathbf{Z}} \mathfrak{g}_{p}$, we will usually denote by $\mathfrak{d}_{k}(\mathfrak{g})$ the centralizer of $\bigoplus_{p \leq-2} \mathfrak{g}_{p}$ in $\mathfrak{g}_{k}$. If $\mathfrak{g}=\bigoplus_{p \in \mathbf{Z}} \mathfrak{g}_{p}$ is an infinite dimensional TGLA of finite depth, then $\mathfrak{d}_{k}(\mathfrak{g}) \neq\{0\}$ for all $k \geq 0$ (see [Tan70. Cor.1 to Theorem 1.11]).

A GLA $\mathfrak{g}=\bigoplus_{p \in \mathbf{Z}} \mathfrak{g}_{p}$ is called completely reducible (resp. irreducible) if the $\mathfrak{g}_{0}$-module $\mathfrak{g}_{-1}$ is so. We abbreviate completely reducible TGLA to c.r.t.-GLA.

An FGLA $\mathfrak{m}=\bigoplus_{p<0} \mathfrak{g}_{p}$ is called nondegenerate if, for $x \in \mathfrak{g}_{-1},\left[x, \mathfrak{g}_{-1}\right]=\{0\}$ implies $x=0$.

Let $\mathfrak{g}=\bigoplus_{p \in \mathbf{Z}} \mathfrak{g}_{p}$ be a TGLA of finite depth. We denote by $\widehat{\mathfrak{g}}=\bigoplus_{p \in \mathbf{Z}} \widehat{\mathfrak{g}}_{p}$ the graded ideal $\bigoplus_{p \neq 0} \mathfrak{g}_{p} \bigoplus\left[\mathfrak{g}_{-1}, \mathfrak{g}_{1}\right]$ of $\mathfrak{g}$ and denote by $\mathfrak{g}^{a}=\bigoplus_{p \in \mathbf{Z}} \mathfrak{g}_{p}^{a}$ the graded ideal $\widehat{\mathfrak{g}}+\left[\mathfrak{g}_{0}, \mathfrak{g}_{0}\right]$ of $\mathfrak{g}$.

Finally given a GLA $\mathfrak{g}=\bigoplus_{p \in \mathbf{Z}} \mathfrak{g}_{p}$ and an integer $k$, we set $\operatorname{Der}_{k}(\mathfrak{g})=\operatorname{Der}(\mathfrak{g}) \cap$ 
$\operatorname{End}_{k}(\mathfrak{g}) ;$ then $\operatorname{Der}(\mathfrak{g})=\bigoplus_{k \in \mathbf{Z}} \operatorname{Der}_{k}(\mathfrak{g})$ if $\operatorname{dim} \mathfrak{g}<\infty$.

1.2. Local Lie algebras (see [Kac68, page 1276-1277]).

A direct sum of vector spaces $\mathfrak{g}_{-1} \bigoplus \mathfrak{g}_{0} \bigoplus \mathfrak{g}_{1}$ is called a local Lie algebra if one has skew-symmetric bilinear mappings [ , ] : $\mathfrak{g}_{i} \times \mathfrak{g}_{j} \rightarrow \mathfrak{g}_{i+j}$ for $|i|,|j|, \mid i+$ $j \mid \leq 1$ such that the Jacobi identity holds whenever it makes sense. Ideals and isomorphisms of local Lie algebras are defined as in the case of GLAs. Given a GLA $\mathfrak{g}=\bigoplus_{p \in \mathbf{Z}} \mathfrak{g}_{p}$, the subspace $\mathfrak{g}_{l o c}:=\mathfrak{g}_{-1} \bigoplus \mathfrak{g}_{0} \bigoplus \mathfrak{g}_{1}$ of $\mathfrak{g}$ is a local Lie algebra, which is called the local part of $\mathfrak{g}$.

Now let $\mathfrak{g}=\bigoplus_{p \in \mathbf{Z}} \mathfrak{g}_{p}$ be a GLA generated by $\mathfrak{g}_{l o c}$. Then the GLA $\mathfrak{g}$ is called minimal if, for any other GLA $\mathfrak{g}^{\prime}$, an isomorphism of the local part of $\mathfrak{g}_{\text {loc }}$ onto $\mathfrak{g}_{\text {loc }}^{\prime}$ extends to an epimorphism of $\mathfrak{g}$ onto $\mathfrak{g}^{\prime}$. Indeed, for any local Lie algebra $\widetilde{\mathfrak{g}}$, there is a minimal GLA $\mathfrak{g}$ whose local part is isomorphic to $\widetilde{\mathfrak{g}}$ (see [Kac68, page 1278]).

1.3. The prolongation of truncated transitive graded Lie algebras.

Here we define a truncated graded Lie algebra (truncated GLA) and its prolongation ([Mor88]). Let $k \in \mathbf{Z}$. A graded vector space $\bigoplus_{p \leq k} \mathfrak{g}_{p}$ is called a truncated GLA of order $k$ if one has a bracket operation (skew-symmetric bilinear mapping) $[, \quad]: \mathfrak{g}_{p} \times \mathfrak{g}_{q} \rightarrow \mathfrak{g}_{p+q}$ for $p, q, p+q \leq k$ such that the Jacobi identity holds whenever it makes sense. A graded ideal of a truncated GLA, a truncated transitive GLA (truncated TGLA) and a truncated GLA of finite depth are defined as in the case of GLAs. Note that a truncated TGLA of order -1 of finite depth is an FGLA. If $\mathfrak{g}=\bigoplus_{p \in \mathbf{Z}} \mathfrak{g}_{p}$ is a GLA, then for each integer $k, \mathfrak{g}(k):=\bigoplus_{p \leq k} \mathfrak{g}_{p}$ becomes a truncated GLA of order $k$ with respect to the induced bracket operation.

Let $\bigoplus_{p \leq k} \mathfrak{g}_{p}$ be a truncated TGLA of finite depth of order $k \geq-1$. Then there is a TGLA $\mathfrak{h}=\bigoplus_{p \in \mathbf{Z}} \mathfrak{h}_{p}$ of finite depth such that:

(1) $\bigoplus_{p \leq k} \mathfrak{h}_{p}=\bigoplus_{p \leq k} \mathfrak{g}_{p}$

(2) $\mathfrak{h}=\bigoplus_{p \in \mathbf{Z}} \mathfrak{h}_{p}$ is maximum among TGLAs satisfying the condition (1) ([Tan70]). The TGLA $\mathfrak{h}=\bigoplus_{p \in \mathbf{Z}} \mathfrak{h}_{p}$ is called the prolongation of $\bigoplus_{p \leq k} \mathfrak{g}_{p} \cdot \mathfrak{h}=$ $\bigoplus_{p \in \mathbf{Z}} \mathfrak{h}_{p}$ will be often denoted by $\operatorname{Prol}\left(\bigoplus_{p \leq k} \mathfrak{g}_{p}\right)$. Note that given an FGLA $\mathfrak{m}=$ $\bigoplus_{p<0} \mathfrak{g}_{p}, \operatorname{Prol}(\mathfrak{m})_{0}\left(=\mathfrak{h}_{0}\right)$ is identified with $\operatorname{Der}_{0}(\mathfrak{m})$.

Let $\bigoplus_{p \leq k} \mathfrak{g}_{p}(k \geq 0)$ be a truncated TGLA of finite depth and $\mathfrak{g}^{(i)}(i=$ $1, \ldots, l)$ be graded ideals of a truncated TGLA $\bigoplus_{p \leq k} \mathfrak{g}_{p}$ such that $\bigoplus_{p \leq k} \mathfrak{g}_{p}$ is a direct sum of graded ideals $\mathfrak{g}^{(i)}$. Then each Prol $\left(\mathfrak{g}^{(i)}\right)$ can be considered as a graded ideal of Prol $\left(\bigoplus_{p \leq k} \mathfrak{g}_{p}\right)$ and $\operatorname{Prol}\left(\bigoplus_{p \leq k} \mathfrak{g}_{p}\right)$ is a direct sum of graded ideals $\operatorname{Prol}\left(\mathfrak{g}^{(i)}\right)$.

1.4. Graded Lie algebras of Cartan type.

In this subsection, following [Kac68] and [Kac70], we describe Lie algebras of Cartan type and their several gradations.

Let $A(m)$ denote the monoid (under addition) of all $m$-tuples of non-negative integers. For $1 \leq i \leq m$, let $\varepsilon_{i}$ be the $m$-tuple $\left(\delta_{1 i}, \ldots, \delta_{m i}\right)$. For an $m$-tuple 
$\mathbf{s}$ of positive integers and $\alpha \in A(m)$, we set $\|\alpha\|_{\mathbf{s}}=\sum_{i=1}^{m} s_{i} \alpha_{i}$. For $m_{i}$-tuples $\mathbf{s}^{(i)}=\left(s_{1}^{(i)}, \ldots, s_{m_{i}}^{(i)}\right)(i=1, \ldots, n)$ of positive integers, we denote by $\left(\mathbf{s}^{(1)}, \ldots, \mathbf{s}^{(n)}\right)$ the $\sum_{i=1}^{n} m_{i}$-tuple $\left(s_{1}^{(1)}, \ldots, s_{m_{1}}^{(1)}, \ldots, s_{m_{n}}^{(n)}, \ldots, s_{m_{n}}^{(n)}\right.$ ). Further we write $\mathbf{1}_{m}$ (or $\mathbf{1}$ if no confusion arises) for the $m$-tuple $(1, \ldots, 1)$, and for a positive integer $k$ we denote the $m$-tuple $(k, \ldots, k)$ by $k \mathbf{1}$. Let $\mathfrak{U}(m)=F\left[x_{1}, \ldots, x_{n}\right]$. For any $m$-tuple $\mathbf{s}$ of positive integers, we define a gradation of $\mathfrak{U}(m)$ by $\mathfrak{U}(m ; \mathbf{s})_{p}=\left\{\sum a_{\alpha} x^{\alpha}:\|\alpha\|_{\mathbf{s}}=\right.$ $\left.p, a_{\alpha} \in F\right\}$, where $x^{\alpha}=x_{1}^{\alpha_{1}} \ldots x_{m}^{\alpha_{m}}$.

Let $W(m ; F)$ be the Lie algebra of all the polynomial vector fields (i.e., $\left.W(m ; F)=\left\{\sum_{i=1}^{m} P_{i} \partial / \partial x_{i}: P_{i} \in \mathfrak{U}(m)\right\}\right)$. For an $m$-tuple $\mathbf{s}$ of positive integers, we set $W(m ; \mathbf{s} ; F)_{p}=\sum_{i=1}^{m} \mathfrak{U}(m ; \mathbf{s})_{p+s_{i}} \partial / \partial x_{i}$.

Consider the following differential forms

$$
\begin{aligned}
& \omega_{S}=d x_{1} \wedge \cdots \wedge d x_{m}, m \geq 2, \\
& \omega_{H}=\sum_{i=1}^{n} d x_{i} \wedge d x_{i+n}, m=2 n, \\
& \omega_{K}=d x_{2 n+1}-\sum_{i=1}^{n} x_{i+n} d x_{i}, m=2 n+1 .
\end{aligned}
$$

Define

$$
\begin{aligned}
& S(n ; F)=\left\{D \in W(n ; F): D \omega_{S}=0\right\}, \\
& C S(n ; F)=\left\{D \in W(n ; F): D \omega_{S} \in F \omega_{S}\right\}, \\
& H(n ; F)=\left\{D \in W(2 n ; F): D \omega_{H}=0\right\}, \\
& C H(n ; F)=\left\{D \in W(2 n ; F): D \omega_{H} \in F \omega_{H}\right\}, \\
& K(n ; F)=\left\{D \in W(2 n+1 ; F): D \omega_{K} \in \mathfrak{U}(2 n+1) \omega_{K}\right\} .
\end{aligned}
$$

(Here the action of $D$ on the differential forms is extended from its action on $\mathfrak{U}(m)$ by requiring that $D$ be a derivation of exterior algebra satisfying $D(d f)=d(D f)$, where $d f=\sum \partial f / \partial x_{i} d x_{i}, f \in \mathfrak{U}(m)$.) These Lie algebras are called Lie algebras of Cartan type. Notice the following facts: (i) $X(n ; F)$ is simple for $X=W, S, H, K$; (ii) $\mathfrak{z}(X(n ; F))=\{0\}$ for $X=C S, C H$; (iii) $[C S(n ; F), C S(n ; F)]=S(n ; F)$, $[C H(n ; F), C H(n ; F)]=H(n ; F)$.

For $n$-tuples $\mathbf{s}=\left(s_{1}, \ldots, s_{n}\right)$ and $\mathbf{t}=\left(t_{1}, \ldots, t_{n}\right)$ of positive integers such that $1=s_{1} \leq \ldots \leq s_{n}$ and $1=t_{1} \leq \ldots \leq t_{n}$, and an integer $\mu \geq 2$ such that $t_{i} \leq[\mu / 2]$ for all $i$, we can define gradations on Lie algebras of Cartan type as follows:

$$
\begin{aligned}
& W(n ; F)=\bigoplus_{p \in \mathbf{Z}} W(n ; \mathbf{s} ; F)_{p}, \\
& S(n ; F)=\bigoplus_{p \in \mathbf{Z}} S(n ; \mathbf{s} ; F)_{p}, S(n ; \mathbf{s} ; F)_{p}=W(n ; \mathbf{s} ; F)_{p} \cap S(n ; F), \\
& C S(n ; F)=\bigoplus_{p \in \mathbf{Z}} C S(n ; \mathbf{s} ; F)_{p}, C S(n ; \mathbf{s} ; F)_{p}=W(n ; \mathbf{s} ; F)_{p} \cap C S(n ; F),
\end{aligned}
$$




$$
\begin{aligned}
& H(n ; F)=\bigoplus_{p \in \mathbf{Z}} H(n ; \mathbf{t} ; \mu ; F)_{p}, H(n ; \mathbf{t} ; \mu ; F)_{p}=W(2 n ; \mathbf{u} ; F)_{p} \cap H(n ; F), \\
& C H(n ; F)=\bigoplus_{p \in \mathbf{Z}} C H(n ; \mathbf{t} ; \mu ; F)_{p}, C H(n ; \mathbf{t} ; \mu ; F)_{p}=W(2 n ; \mathbf{u} ; F)_{p} \cap C H(n ; F), \\
& K(n ; F)=\bigoplus_{p \in \mathbf{Z}} K(n ; \mathbf{t} ; \mu ; F)_{p}, K(n ; \mathbf{t} ; \mu ; F)_{p}=W(2 n+1 ; \mathbf{v} ; F)_{p} \cap K(n ; F),
\end{aligned}
$$

where $\mathbf{u}=\left(t_{1}, \ldots, t_{n}, \mu-t_{1}, \ldots, \mu-t_{n}\right)$ and $v=\left(t_{1}, \ldots, t_{n}, \mu-t_{1}, \ldots, \mu-t_{n}, \mu\right)$. The GLAs $W(n), S(n), C S(n), H(n), C H(n), K(n)$ equipped with the above gradation will be denoted by $W(n ; \mathbf{s} ; F), S(n ; \mathbf{s} ; F), C S(n ; \mathbf{s} ; F), H(n ; \mathbf{t} ; \mu ; F)$, $C H(n ; \mathbf{t} ; \mu ; F), K(n ; \mathbf{t} ; \mu ; F)$ respectively. These GLAs become TGLAs of finite depth. In particular, when $\mathbf{s}=\mathbf{t}=\mathbf{1}$ and $\mu=2$, the above gradation is called the standard gradation, and the GLAs become infinite dimensional irreducible TGLAs of finite depth. Moreover for a positive integer $m$, we define graded subalgebras $\mathfrak{d}(m ; F)=\bigoplus_{p \in \mathbf{Z}} \mathfrak{d}(m ; F)_{p}$ and $\mathfrak{c} \mathfrak{d}(m ; F)=\bigoplus_{p \in \mathbf{Z}} \mathfrak{c} \mathfrak{d}(m ; F)_{p}$ of $K(m ; \mathbf{1} ; 2 ; F)$ as follows: $\mathfrak{d}(m ; F)=\left\{D \in K(m ; F): D \omega_{K}=0\right\}, \mathfrak{c d}(m ; F)=\{D \in K(m ; F)$ : $\left.D \omega_{K} \in F \omega_{K}\right\}, \mathfrak{d}(m ; F)_{p}=\mathfrak{d}(m ; F) \cap K(n ; \mathbf{1} ; 2 ; F)_{p}, \mathfrak{c} \mathfrak{d}(m ; F)_{p}=\mathfrak{c d}(m ; F) \cap$ $K(m ; \mathbf{1} ; 2 ; F)_{p}$. Then the GLAs $\mathfrak{d}(m ; F)$ and $\mathfrak{c} \mathfrak{d}(m ; F)$ become infinite dimensional irreducible TGLAs of finite depth. Note that $\mathfrak{z}(\mathfrak{d}(m ; F))=\mathfrak{d}(m ; F)_{-2}$ and $[\mathfrak{c} \mathfrak{d}(m ; F)$, $\mathfrak{c} \mathfrak{d}(m ; F)]=\mathfrak{d}(m ; F)$. For the sake of simplicity, we denote by $X(n ; \mathbf{s})$ (resp. $Y(n ; \mathbf{t} ; \mu)$, or $Z(n ; F))$ the GLA $X(n ; \mathbf{s} ; \mathbf{C})(\operatorname{resp} . Y(n ; \mathbf{t} ; \mu ; \mathbf{C})$, or $Z(n ; F))$ for $X=W, S, C S(\operatorname{resp} . Y=H, C H, K$, or $Z=\mathfrak{d}, \mathfrak{c} \mathfrak{d})$.

\subsection{Pseudo-product GLAs (see [Tan85]).}

Let $\mathfrak{g}=\bigoplus_{p \in \mathbf{Z}} \mathfrak{g}_{p}$ be a TGLA of finite depth and $\mathfrak{e}^{1}$ and $\mathfrak{e}^{2}$ be two $\mathfrak{g}_{0^{-}}$ submodules of $\mathfrak{g}_{-1}$. Then the system $\mathfrak{G}=\left(\mathfrak{g},\left(\mathfrak{g}_{p}\right)_{p \in \mathbf{Z}}, \mathfrak{e}^{1}, \mathfrak{e}^{2}\right)$ is called a pseudoproduct GLA if the following conditions hold:

(1) $\mathfrak{g}_{-1}=\mathfrak{e}^{1} \bigoplus \mathfrak{e}^{2}$

(2) $\left[\mathfrak{e}^{i}, \mathfrak{e}^{i}\right]=\{0\} \quad(i=1,2)$.

In particular, if $\mathfrak{g}$ is an FGLA, then $\mathfrak{G}$ is called a pseudo-product fundamental GLA (pseudo-product FGLA). Given a pseudo-product GLA $\mathfrak{G}=\left(\mathfrak{g},\left(\mathfrak{g}_{p}\right)_{p \in \mathbf{Z}}, \mathfrak{e}^{1}, \mathfrak{e}^{2}\right)$, the system $\mathfrak{G}_{-}:=\left(\mathfrak{g}_{-},\left(\mathfrak{g}_{p}\right)_{p<0}, \mathfrak{e}^{1}, \mathfrak{e}^{2}\right)$ is a pseudo-product FGLA, which is called the negative part of $\mathfrak{G}$. Moreover a pseudo-product GLA $\mathfrak{G}=\left(\mathfrak{g},\left(\mathfrak{g}_{p}\right)_{p \in \mathbf{Z}}, \mathfrak{e}^{1}, \mathfrak{e}^{2}\right)$ is said to be of irreducible type if each $\mathfrak{e}^{i}$ is an irreducible $\mathfrak{g}_{0}$-module and $\mathfrak{g}_{-2} \neq\{0\}$.

Let $\mathfrak{G}=\left(\mathfrak{g},\left(\mathfrak{g}_{p}\right)_{p \in \mathbf{Z}}, \mathfrak{e}^{1}, \mathfrak{e}^{2}\right)$ and $\mathfrak{G}^{\prime}=\left(\mathfrak{g}^{\prime},\left(\mathfrak{g}_{p}^{\prime}\right)_{p \in \mathbf{Z}}, \mathfrak{e}^{\prime 1}, \mathfrak{e}^{\prime 2}\right)$ be two pseudoproduct GLAs. By an isomorphism of $\mathfrak{G}$ onto $\mathfrak{G}^{\prime}$, we mean an isomorphism $\phi$ of $\mathfrak{g}$ onto $\mathfrak{g}^{\prime}$ such that $\phi\left(\mathfrak{g}_{p}\right)=\mathfrak{g}_{p}^{\prime}$ for all $p \in \mathbf{Z}$ and $\phi\left(\mathfrak{e}^{i}\right)=\mathfrak{e}^{\prime \sigma(i)}$ for some permutation $\sigma$ of order 2 .

A pseudo-product GLA $\mathfrak{G}=\left(\mathfrak{g},\left(\mathfrak{g}_{p}\right)_{p \in \mathbf{Z}}, \mathfrak{e}^{1}, \mathfrak{e}^{2}\right)$ is said to be of depth $\mu$ (resp. completely reducible, semisimple) if the underlying GLA $\mathfrak{g}=\bigoplus_{p \in \mathbf{Z}} \mathfrak{g}_{p}$ is so.

Let $\mathfrak{M}=\left(\mathfrak{m},\left(\mathfrak{g}_{p}\right)_{p<0}, \mathfrak{e}^{1}, \mathfrak{e}^{2}\right)$ be a pseudo-product FGLA and $\mathfrak{g}_{0}$ be the derivation algebra of $\mathfrak{M}$ (i.e., $\left.\mathfrak{g}_{0}=\left\{D \in \operatorname{Der}_{0}\left(\mathfrak{g}_{-}\right):\left[D, \mathfrak{e}^{i}\right] \subset \mathfrak{e}^{i}(i=1,2)\right\}\right)$. Further let $\stackrel{\vee}{\mathfrak{g}}=$ 
$\bigoplus_{p \in \mathbf{Z}} \stackrel{\vee}{\mathfrak{g}_{p}}$ be the prolongation of $\mathfrak{m} \bigoplus \stackrel{\vee}{\mathfrak{g}_{0}}$. Then the system $\stackrel{\vee}{\mathfrak{M}}=\left(\stackrel{\vee}{\mathfrak{g}},\left(\stackrel{\vee}{\mathfrak{g}_{p}}\right)_{p \in \mathbf{Z}}, \mathfrak{e}^{1}, \mathfrak{e}^{2}\right)$ is a pseudo-product GLA, which is called the prolongation of $\mathfrak{M}$.

Let $\mathfrak{G}=\left(\mathfrak{g},\left(\mathfrak{g}_{p}\right)_{p \in \mathbf{Z}}, \mathfrak{e}^{1}, \mathfrak{e}^{2}\right)$ be a pseudo-product GLA of depth $\mu$. Following $\mathrm{N}$. Tanaka, $\mathfrak{G}$ is called a pseudo-projective GLA of order $\mu$ of bidegree $(n, r)$ if the following conditions hold:

(1) $\mathfrak{g}_{-}$is non-degenerate;

(2) $\mathfrak{e}^{2} \bigoplus \bigoplus_{p \leq-2} \mathfrak{g}_{p}$ is a commutative subspace of $\mathfrak{g}$;

(3) For $x \in \mathfrak{g}_{p}(-\mu+1 \leq p \leq-2),\left[x, \mathfrak{e}^{1}\right]=\{0\}$ implies $x=0$.

(4) $\operatorname{dim} \mathfrak{e}^{1}=r$ and $\operatorname{dim} \mathfrak{g}_{-\mu}=n-r$.

(5) $\operatorname{dim} \mathfrak{g}_{p}=(n-r)_{r} H_{\mu+p}$ for $-\mu+1 \leq p \leq-2$ and $\operatorname{dim} \mathfrak{e}^{2}=(n-r)_{r} H_{\mu-1}$. Also pseudo-projective FGLAs of order $\mu$ of bidegree $(n, r)$ are defined as in the case of pseudo-product FGLAs.

Let $\mathfrak{G}=\left(\mathfrak{g},\left(\mathfrak{g}_{p}\right)_{p \in \mathbf{Z}}, \mathfrak{e}^{1}, \mathfrak{e}^{2}\right)$ be a pseudo-projective GLA of order $\mu$ of bidegree $(n, r)$. Now we consider the following graded vector space: $\mathfrak{c}=\bigoplus_{p<0} \mathfrak{c}_{p}$, where $\mathfrak{c}_{p}=\mathfrak{g}_{-\mu} \otimes S^{p+\mu}\left(\mathfrak{e}^{1 *}\right)(-\mu \leq p \leq-2), \mathfrak{c}_{-1}=\mathfrak{e}^{1} \bigoplus \mathfrak{t}, \mathfrak{t}=\mathfrak{g}_{-\mu} \otimes S^{\mu-1}\left(\mathfrak{e}^{1 *}\right)$ and $\mathfrak{c}_{p}=\{0\}(p<-\mu)$. Then $\mathfrak{c}$ naturally becomes an FGLA, which is called the contact algebra of $(\mu+1)$-th order of bidegree $(r, n-r)$ (see [Yam82, page 133-134]). Further the system $\mathfrak{C}=\left(\mathfrak{c},\left(\mathfrak{c}_{p}\right)_{p<0}, \mathfrak{e}^{1}, \mathfrak{t}\right)$ is a pseudo-projective FGLA of order $\mu$ of bidegree $(n, r)$ and isomorphic to the negative part $\mathfrak{G}_{-}$of $\mathfrak{G}$. Consequently the prolongation $\mathfrak{G}$ of $\mathfrak{G}_{\text {_ }}$ coincides with that of $\mathfrak{C}$. In other words, there is a unique pseudo-projective GLA of order $\mu$ of bidegree $(n, r)$ up to isomorphism such that $\mathfrak{G}=\mathfrak{G}$. Also the prolongation of $\mathfrak{g}_{-}$is isomorphic to $K(r ; \mathbf{1} ; \mu)$ if $n-r=1$, and isomorphic to $W\left(n ;\left(\mathbf{1}_{r}, 2 \mathbf{1}_{n-r}\right)\right)$ if $n-r \neq 1$ (see [Mor88, Cor.1 and Cor.3]).

\section{§2. Complex completely reducible transitive graded Lie algebras of finite depth}

In this section, we investigate the structure of a c.r.t.-GLA of finite depth over $\mathbf{C}$.

2.1. Let $\mathfrak{g}=\bigoplus_{p \in \mathbf{Z}} \mathfrak{g}_{p}$ be a c.r.t.-GLA of finite depth over $\mathbf{C}$. Since the $\mathfrak{g}_{0}$ module $\mathfrak{g}_{-1}$ is completely reducible and faithful, $\mathfrak{g}_{0}$ is reductive. Moreover we can easily prove that the $\mathfrak{g}_{0}$-module $\mathfrak{g}_{1}$ is completely reducible (cf. [Yat88, Lemma 2.1]). We denote by $\mathfrak{g}_{0}^{\prime}$ the semisimple part of $\mathfrak{g}_{0}$. We decompose the $\mathfrak{g}_{0}$-module $\mathfrak{g}_{-1}$ (resp. $\mathfrak{g}_{1}$ ) into a direct sum of irreducible $\mathfrak{g}_{0}$-submodules as follows:

$$
\mathfrak{g}_{-1}=\bigoplus_{s=1}^{n(-1)} \mathfrak{g}_{-1}^{(s)}, \quad \mathfrak{g}_{1}=\bigoplus_{t=1}^{n(1)} \mathfrak{g}_{1}^{(t)}
$$

We set $I_{-1}=\{1, \ldots, n(-1)\}$ and $I_{1}=\{1, \ldots, n(1)\}$. We fix a Cartan subalgebra $\mathfrak{h}$ of $\mathfrak{g}_{0}$ and a simple root system $\Pi=\left\{\alpha_{1}, \ldots, \alpha_{l}\right\}$ of $\mathfrak{g}_{0}^{\prime}$. We denote by $\Lambda_{s}$ (resp. $M_{t}$ ) the highest weight (resp. lowest weight) of the irreducible $\mathfrak{g}_{0}$-module $\mathfrak{g}_{-1}^{(s)}$ (resp. $\mathfrak{g}_{1}^{(t)}$ ) 
and by $F_{\Lambda_{s}}$ (resp. $E_{M_{t}}$ ) the corresponding weight vector. Furthermore we denote by $\phi_{-1}^{(s)}$ (resp. $\phi_{1}^{(t)}$ ) the representation of $\mathfrak{g}_{0}^{\prime}$ on $\mathfrak{g}_{-1}^{(s)}\left(\right.$ resp. $\mathfrak{g}_{1}^{(t)}$ ). For a semisimple ideal $\mathfrak{a}$ of $\mathfrak{g}_{0}^{\prime}$, we denote by $\Delta(\mathfrak{a})$ the set of all the roots of $\mathfrak{a}$. We denote by $(\mid)$ the Killing form of $\mathfrak{g}_{0}^{\prime}$. For $\alpha \in \Delta\left(\mathfrak{g}_{0}^{\prime}\right)$, we denote by $\alpha^{\vee}$ the element $2 \nu^{-1}(\alpha) /(\alpha \mid \alpha)$ of $\mathfrak{h}$ (where $\nu$ is an isomorphism of $\mathfrak{h} \cap \mathfrak{g}_{0}^{\prime}$ onto $\left(\mathfrak{h} \cap \mathfrak{g}_{0}^{\prime}\right)^{*}$ defined by $\langle\nu(x), y\rangle=(x \mid y)$ ) and fix a root vector $e_{\alpha}$ corresponding to the root $\alpha$. In this paper, we will usually assume $\left[e_{\alpha}, e_{-\alpha}\right]=\alpha^{\vee}$ for all $\alpha \in \Delta\left(\mathfrak{g}_{0}^{\prime}\right)$. Moreover we denote by $e_{i}$ (resp. $f_{i}$ ) the root vector $e_{\alpha_{i}}$ (resp. $e_{-\alpha_{i}}$ ).

First of all, we state a criterion for a TGLA to be of finite depth. The following lemma is due to [Kac68, Theorems 1 and 3].

Lemma 1. Let $\mathfrak{g}=\bigoplus_{p \in \mathbf{Z}} \mathfrak{g}_{p}$ be a GLA and let $A=\left(a_{i j}\right)$ be an $l \times l$ matrix with the following properties:

(i) $\quad a_{i i}=2$ or $a_{i i}=0$ for all $i$;

(ii) if $a_{i i}=0$ for some $i$, then for at least one such $i$ there exists a $j$ for which the matrix $\left(\begin{array}{cc}0 & a_{i j} \\ a_{j i} & a_{j j}\end{array}\right)$ contains no zero row;

(iii) $A$ is not the Cartan matrix of a finite dimensional semisimple Lie algebra.

Then $\mathfrak{g}$ is of infinite depth, if there are homogeneous elements $e_{1}^{\prime}, f_{i}^{\prime}, h_{i}^{\prime}(i=$ $1, \ldots, l)$ of $\mathfrak{g}$ satisfying the following conditions:

$$
\left[e_{i}^{\prime}, f_{j}^{\prime}\right]=\delta_{i j} h_{j}^{\prime},\left[h_{i}^{\prime}, e_{j}^{\prime}\right]=a_{i j} e_{j}^{\prime},\left[h_{i}^{\prime}, f_{j}^{\prime}\right]=-a_{i j} f_{j}^{\prime},\left[h_{i}^{\prime}, h_{j}^{\prime}\right]=0
$$

Furthermore the following lemma is also useful, and its proof is easy.

Lemma 2. Let $\mathfrak{g}=\bigoplus_{p \in \mathbf{Z}} \mathfrak{g}_{p}$ be a GLA and $f_{1}^{\prime}, f_{2}^{\prime}, e_{1}^{\prime}$, $h_{1}^{\prime}$ be the elements of $\mathfrak{g}$ such that $f_{1}^{\prime} \in \mathfrak{g}_{-p_{i}}, e_{1}^{\prime} \in \mathfrak{g}_{p_{1}}, h_{1}^{\prime} \in \mathfrak{g}_{0}\left(p_{1}>0, p_{2} \geq 0\right),\left[e_{1}^{\prime}, f_{1}^{\prime}\right]=h_{1}^{\prime}, \quad\left[e_{1}^{\prime}, f_{2}^{\prime}\right]=$ $0,\left[h_{1}^{\prime}, f_{i}^{\prime}\right]=-a_{1 i} f_{i}^{\prime}, a_{1 i} \in \mathbf{C}$. Then $\mathfrak{g}$ is of infinite depth if one of the following conditions holds:

(i) $\quad a_{11} \neq 0, a_{12}=0,\left[f_{1}^{\prime}, f_{2}^{\prime}\right] \neq 0$;

(ii) $a_{12} \neq 0, a_{11}=0$;

(iii) $\quad a_{11} \neq 0,-2 a_{12} / a_{11} \notin \mathbf{Z}$ or $-2 a_{12} / a_{11}<0$.

By transitivity, for each $s \in I_{1}$, there is a $t \in I_{-1}$ such that $\left[\mathfrak{g}_{-1}^{(t)}, \mathfrak{g}_{1}^{(s)}\right] \neq\{0\}$. Note that $\left[\mathfrak{g}_{-1}^{(t)}, \mathfrak{g}_{1}^{(s)}\right] \neq\{0\}$ if and only if $\left[F_{\Lambda_{t}}, E_{M_{s}}\right] \neq\{0\}$. For convenience, we assume that $\left[\mathfrak{g}_{-1}^{(1)}, \mathfrak{g}_{1}^{(1)}\right] \neq\{0\}$. Now we decompose $\mathfrak{g}_{0}^{\prime}$ into a direct sum of semisimple ideals as follows:

$$
\mathfrak{g}_{0}^{\prime}=\mathfrak{a}_{1} \oplus \mathfrak{a}_{2} \oplus \mathfrak{a}_{3} \oplus \mathfrak{a}_{4}, \mathfrak{a}_{1} \oplus \mathfrak{a}_{2}=\operatorname{Ker} \phi_{-1}^{(1)}, \mathfrak{a}_{2} \oplus \mathfrak{a}_{3}=\operatorname{Ker} \phi_{1}^{(1)}
$$

Moreover we set $I_{0 i}=\left\{j: \alpha_{j} \in \Delta\left(\mathfrak{a}_{i}\right)\right\}$. 
Now we investigate the relation between $\mathfrak{g}_{-1}^{(1)}$ and $\mathfrak{g}_{1}^{(1)}$ when $\mathfrak{g}_{-1}^{(1)}$ is contragredient to $\mathfrak{g}_{1}^{(1)}$ as a $\mathfrak{g}_{0}$-module (i.e., $\Lambda_{1}+M_{1}=0$ ).

LeMma 3. Suppose that $\Lambda_{1}+M_{1}=0$ and set $h:=\left[E_{M_{1}}, F_{\Lambda_{1}}\right]$.

(1) $\left\langle M_{1}, h\right\rangle \neq 0$.

(2) $\Lambda_{s}+M_{1} \neq 0$ for all $s \in I_{-1} \backslash\{1\}$.

(3) $\mathfrak{a}_{1}=\mathfrak{a}_{3}=\{0\}$ and $h \in \mathfrak{z}\left(\mathfrak{g}_{0}\right) \bigoplus \mathfrak{a}_{4}$.

Proof. (1) We now suppose that $\left[h, E_{M_{1}}\right]=0$. If $h \notin \mathfrak{z}\left(\mathfrak{g}_{0}\right)$, then there is a positive root $\alpha$ of $\mathfrak{g}_{0}^{\prime}$ such that $\langle\alpha, h\rangle \neq 0$. Applying Lemma 2 to the case when $e_{1}^{\prime}=E_{M_{1}}, f_{1}^{\prime}=F_{\Lambda_{1}}, h_{1}^{\prime}=h, f_{2}^{\prime}=e_{-\alpha}$, we can reach a contradiction. Thus it suffices to consider the case when $h \in \mathfrak{z}\left(\mathfrak{g}_{0}\right)$. By transitivity, there is an $i \in I_{-1}$ such that $\left[h, F_{\Lambda_{i}}\right] \neq 0$. If $\left[E_{M_{1}}, F_{\Lambda_{i}}\right] \neq 0$, then $0=\left[h,\left[E_{M_{1}}, F_{\Lambda_{i}}\right]\right]=\left\langle\Lambda_{i}, h\right\rangle\left[E_{M_{1}}, F_{\Lambda_{i}}\right]$, which is a contradiction. If $\left[E_{M_{1}}, F_{\Lambda_{i}}\right]=0$, then we can reach a contradiction by applying Lemma 2 to the case when $e_{1}^{\prime}=E_{M_{1}}, f_{1}^{\prime}=F_{\Lambda_{1}}, f_{2}^{\prime}=F_{\Lambda_{i}}, h_{1}^{\prime}=h$.

(2) The proof of this assertion is the same as the proof of Lemma 1.4 in [Yat88].

(3) Since $\Lambda_{1}+M_{1}=0$, we have $\mathfrak{a}_{1}=\mathfrak{a}_{3}=\{0\}$. By (1), we may assume that $\left\langle M_{1}, h\right\rangle=2$. If $h \notin \mathfrak{z}\left(\mathfrak{g}_{0}\right) \bigoplus \mathfrak{a}_{4}$, then there is a $k \in I_{02}$ such that $\left\langle\alpha_{k}, h\right\rangle \neq 0$. Consider the elements $e_{1}^{\prime}=E_{M_{1}}, e_{2}^{\prime}=e_{k}, f_{1}^{\prime}=F_{\Lambda_{1}}, f_{2}^{\prime}=f_{k}, h_{1}^{\prime}=h, h_{2}^{\prime}=\alpha_{k}^{\vee}$; these elements satisfy the relation (2.1). Since $\left[\alpha_{k}^{\vee}, E_{M_{1}}\right]=0$ and $\left\langle\alpha_{k}, h\right\rangle \neq 0$, it is impossible by Lemma 1 . Hence $h \in \mathfrak{z}\left(\mathfrak{g}_{0}\right) \bigoplus \mathfrak{a}_{4}$.

Next we investigate the relation between $\mathfrak{g}_{-1}^{(1)}$ and $\mathfrak{g}_{1}^{(1)}$ when $\mathfrak{g}_{-1}^{(1)}$ is not contragredient to $\mathfrak{g}_{1}^{(1)}$ as a $\mathfrak{g}_{0}$-module. Also, when $\Lambda_{1}+M_{1}=-\alpha \neq 0$ and $\left[E_{M_{1}}, F_{\Lambda_{1}}\right] \neq 0$, we will assume that $\left[E_{M_{1}}, F_{\Lambda_{1}}\right]=e_{-\alpha}$, rescaling, if necessary, the elements $e_{\alpha}, e_{-\alpha}$ : $e_{\alpha} \rightarrow c e_{\alpha}, e_{-\alpha} \rightarrow c^{-1} e_{-\alpha}$, where $c \in \mathbf{C}^{\times}$.

Lemma 4. Suppose that $\Lambda_{1}+M_{1}=-\alpha \neq 0$. Then $\mathfrak{a}_{1}=\mathfrak{a}_{3}=\{0\}, \mathfrak{a}_{4}=$ $\left[\mathfrak{g}_{-1}^{(1)}, \mathfrak{g}_{1}^{(1)}\right], \mathfrak{a}_{4}$ is a simple Lie algebra of type $A_{l}$ or $C_{l}$, the restriction of $\phi_{-1}^{(1)}$ to $\mathfrak{a}_{2}$ is the elementary representation and $\alpha$ is the highest root $\theta_{4}$ of $\mathfrak{a}_{4}$.

Proof. First we suppose that $\alpha \in \Delta\left(\mathfrak{a}_{3}\right)$. Then $\mathfrak{a}_{3}$ is simple. Indeed, if there is a simple component $\mathfrak{b}$ of $\mathfrak{a}_{3}$ such that $\alpha \notin \Delta(\mathfrak{b})$, then $\left\langle\Lambda_{1}, \theta^{\vee}\right\rangle=-\left\langle M_{1}, \theta^{\vee}\right\rangle-$ $\left\langle\alpha, \theta^{\vee}\right\rangle=0$, where $\theta$ is the highest root of $\mathfrak{b}$. This is a contradiction. Clearly $-\alpha$ is the highest root of $\mathfrak{a}_{3}$, which we write $\alpha=-\theta_{3}$. Since $\alpha \in \Delta\left(\mathfrak{a}_{3}\right)$, we have $\left\langle M_{1}, \theta_{3}^{\vee}\right\rangle=0$. Hence we can reach a contradiction by applying Lemma 2 to the case when $f_{1}^{\prime}=\left[F_{\Lambda_{1}}, e_{-\theta_{3}}\right], f_{2}^{\prime}=e_{-\theta_{3}}, h_{1}^{\prime}=\theta_{3}^{\vee}, e_{1}^{\prime}=E_{M_{1}}$. Secondly suppose that $\alpha \in \Delta\left(\mathfrak{a}_{2}\right)$. Then $\left\langle\Lambda_{1}, \alpha^{\vee}\right\rangle=\left\langle M_{1}, \alpha^{\vee}\right\rangle=0$ and $\left\langle\alpha, \alpha^{\vee}\right\rangle \neq 0$, which is a contradiction. Thirdly suppose that $\alpha \in \Delta\left(\mathfrak{a}_{4}\right)$. Then $\mathfrak{a}_{1}=\mathfrak{a}_{3}=\{0\}$. Indeed, if $\mathfrak{a}_{1} \neq\{0\}$ (resp. $\left.\mathfrak{a}_{3} \neq\{0\}\right)$, then $\left\langle\Lambda_{1}, \theta_{1}^{\vee}\right\rangle=0$ and $\left\langle M_{1}, \theta_{1}^{\vee}\right\rangle \neq 0\left(\right.$ resp. $\left\langle\Lambda_{1}, \theta_{3}^{\vee}\right\rangle \neq 0$ and $\left.\left\langle M_{1}, \theta_{3}^{\vee}\right\rangle=0\right)$ for the highest weight $\theta_{1}$ (resp. $\theta_{3}$ ) of a semisimple component of $\mathfrak{a}_{1}$ (resp. $\mathfrak{a}_{3}$ ), which is a contradiction. In this case, since $\mathfrak{g}$ is of finite depth, the results of the lemma 
follows from [Kac68, Theorem 2]. Finally we suppose that $\alpha \in \Delta\left(\mathfrak{a}_{1}\right)$. As in the case when $\alpha \in \Delta\left(\mathfrak{a}_{4}\right)$, we have $\mathfrak{a}_{3}=\{0\}$. Also as in the case when $\alpha \in \Delta\left(\mathfrak{a}_{3}\right), \mathfrak{a}_{1}$ is simple and $\alpha$ is the highest root of $\mathfrak{a}_{1}$, which we write $\alpha=\theta_{1}$. Choose $\mathfrak{g}_{-1}^{(t)}$ such that $\operatorname{Ker} \phi_{-1}^{(t)} \not \supset \mathfrak{a}_{1}$. For convenience, we assume $t=2$. If $\left[\mathfrak{g}_{-1}^{(2)}, \mathfrak{g}_{1}^{(1)}\right] \neq\{0\}$ and $\Lambda_{2}+M_{1}=$ 0 , then $\left\langle M_{1}, \alpha_{i}^{\vee}\right\rangle=-\left\langle\theta_{1}, \alpha_{i}^{\vee}\right\rangle$ for $i \in I_{01}$. Consider the elements $e_{0}^{\prime}=E_{M_{1}}, h_{0}^{\prime}=$ $\left[E_{M_{1}}, F_{\Lambda_{2}}\right], f_{0}^{\prime}=F_{\Lambda_{2}}, e_{i}^{\prime}=e_{i}, h_{i}^{\prime}=\alpha_{i}^{\vee}, f_{i}^{\prime}=f_{i}\left(i \in I_{01}\right)$; these elements satisfy the relation (2.1). Since the corresponding matrix $\left(a_{i j}\right)$ is the Cartan matrix of an affine Lie algebra, we can reach a contradiction by Lemma 1 . If $\left[\mathfrak{g}_{-1}^{(2)}, \mathfrak{g}_{1}^{(1)}\right] \neq$ $\{0\}$ and $\Lambda_{2}+M_{1}=\beta \neq 0$, then, by the above results, $\beta \in \Delta\left(\mathfrak{a}_{1} \bigoplus \mathfrak{s}_{4} \bigoplus \mathfrak{t}_{4}\right)$. (Here we decompose $\mathfrak{a}_{2}$ and $\mathfrak{a}_{4}$ into a direct sum of simple ideals as follows: $\mathfrak{a}_{2}=$ $\left.\mathfrak{s}_{2} \bigoplus \mathfrak{t}_{2}, \mathfrak{a}_{4}=\mathfrak{s}_{4} \bigoplus \mathfrak{t}_{4}, \operatorname{Ker} \phi_{-1}^{(2)}=\mathfrak{s}_{2} \bigoplus \mathfrak{s}_{4}, \operatorname{Ker} \phi_{1}^{(1)}=\mathfrak{s}_{2} \bigoplus \mathfrak{t}_{2}.\right)$ If $\beta \in \Delta\left(\mathfrak{a}_{1} \bigoplus \mathfrak{t}_{4}\right)$, then $\Lambda_{2}+M_{1}=-\theta_{1}$, so $\Lambda_{1}=\Lambda_{2}$, which is a contradiction. If $\beta \in \Delta\left(\mathfrak{s}_{4}\right)$, then $\mathfrak{s}_{4}$ is simple and $\beta=-\theta^{\prime}$, where $\theta^{\prime}$ is the highest root of $\mathfrak{s}_{4}$. Multiplying, if necessary, $F_{\Lambda_{2}}$ by a nonzero number, we may assume that $\left[E_{M_{1}}, F_{\Lambda_{2}}\right]=e_{-\theta^{\prime}}$. Consider the elements $e_{1}^{\prime}=\left[E_{M_{1}}, e_{\theta_{1}}\right], f_{1}^{\prime}=F_{\Lambda_{1}}, h_{1}^{\prime}=-\theta_{1}^{\vee}, e_{2}^{\prime}=\left[E_{M_{1}}, e_{\theta^{\prime}}\right], f_{2}^{\prime}=F_{\Lambda_{2}}, h_{2}^{\prime}=-\theta^{\prime \vee} ;$ these elements satisfy the relation (2.1). Since $\left\langle\Lambda_{1}, \theta_{1}^{\vee}\right\rangle=\left\langle\Lambda_{2}, \theta^{\prime \vee}\right\rangle=0,\left\langle\Lambda_{1}, \theta^{\prime \vee}\right\rangle>0$ and $\left\langle\Lambda_{2}, \theta_{1}^{\vee}\right\rangle>0$, we can reach a contradiction by Lemma 1 . If we suppose that $\left[\mathfrak{g}_{-1}^{(2)}, \mathfrak{g}_{1}^{(1)}\right]=\{0\}$, then we can reach a contradiction by applying Lemma 2 to the case when $e_{1}^{\prime}=\left[E_{M_{1}}, e_{\theta_{1}}\right], f_{1}^{\prime}=F_{\Lambda_{1}}, f_{2}^{\prime}=F_{\Lambda_{2}}, h_{1}^{\prime}=-\theta_{1}^{\vee}$.

Here we remark that, under the assumption of Lemma $4, \mathfrak{g}_{-1}^{(1)} \bigoplus\left[\mathfrak{g}_{-1}^{(1)}, \mathfrak{g}_{1}^{(1)}\right] \bigoplus$ $\mathfrak{g}_{1}^{(1)}$ is isomorphic, as a local Lie algebra, to the local part of $S(n ; \mathbf{1})$ or $H(n ; \mathbf{1} ; 2)$. In particular, in this case, the subalgebra of $\mathfrak{g}$ generated by $\mathfrak{g}_{-1}^{(1)} \bigoplus\left[\mathfrak{g}_{-1}^{(1)}, \mathfrak{g}_{1}^{(1)}\right] \bigoplus \mathfrak{g}_{1}^{(1)}$ is infinite dimensional.

Lemma 5. Suppose that $\Lambda_{1}+M_{1} \neq 0$. Then $\Lambda_{s}+M_{1} \neq-\theta_{4}$ for all $s \in$ $I_{-1} \backslash\{1\}$.

Proof. Suppose that there is an $s \in I_{-1} \backslash\{1\}$ such that $\Lambda_{s}+M_{1}=-\theta_{4}$. In this case, $\Lambda_{1}=\Lambda_{s}$. There is a complex number $c$ such that $\left[F_{\Lambda_{s}}-c F_{\Lambda_{1}}, E_{M_{1}}\right]=0$. Apply Lemma 2 to the case when $e_{1}^{\prime}=\left[E_{M_{1}}, e_{\theta_{4}}\right], f_{1}^{\prime}=F_{\Lambda_{1}}, f_{2}^{\prime}=F_{\Lambda_{s}}-c F_{\Lambda_{1}}, h_{1}^{\prime}=$ $-\theta_{4}^{\vee}$. Since $\left\langle\Lambda_{1}, \theta_{4}^{\vee}\right\rangle>0$ and $\left\langle\Lambda_{s}, \theta_{4}^{\vee}\right\rangle>0$, we can reach a contradiction.

LEMMA 6. $\quad\left[\mathfrak{g}_{-1}^{(s)}, \mathfrak{g}_{1}^{(1)}\right]=\{0\}$ for all $s \in I_{-1} \backslash\{1\}$.

Proof. Suppose that $\left[\mathfrak{g}_{-1}^{(s)}, \mathfrak{g}_{1}^{(1)}\right] \neq\{0\}$ for some $s \in I_{-1} \backslash\{1\}$. For convenience, we assume that $s=2$. By Lemmas 3 and 5 , we have (1) $\Lambda_{1}+M_{1} \neq 0$, $\Lambda_{2}+M_{1}=0$ or (2) $\Lambda_{1}+M_{1}=0, \Lambda_{2}+M_{1} \neq 0$. We may assume the case (1) (i.e., $\Lambda_{1}+M_{1} \neq 0, \Lambda_{2}+M_{1}=0$ ). By Lemma $4, \Lambda_{1}+M_{1}=-\theta_{4}, \mathfrak{g}_{0}^{\prime}=$ $\mathfrak{a}_{2} \bigoplus \mathfrak{a}_{4}, \mathfrak{a}_{4}$ is a simple Lie algebra of type $A_{l}$ or $C_{l}$ and $\Lambda_{1}$ is the highest weight of the elementary representation of type $A_{l}$ or $C_{l}$. We set $e_{0}=E_{M_{1}}, f_{0}=F_{\Lambda_{2}}$, $h_{0}=\left[E_{M_{1}}, F_{\Lambda_{2}}\right]$. Here we may assume that $\left[h_{0}, e_{0}\right]=2 e_{0}$. Consider the ele- 
ments $e_{0}^{\prime}=e_{0}, f_{0}^{\prime}=f_{0}, h_{0}^{\prime}=h_{0}, e_{i}^{\prime}=e_{i}, f_{i}^{\prime}=f_{i}, h_{i}^{\prime}=\alpha_{i}^{\vee}\left(i \in I_{04}\right)$; these elements satisfy the relation (2.1). By Lemma 1 , only the case when $\mathfrak{a}_{4}$ is of type $A_{1}$ is possible. Then we have $\left\langle M_{1}, h_{i_{0}}^{\prime}\right\rangle=-3$, so $\left\langle\alpha_{i_{0}}, h_{0}^{\prime}\right\rangle=-1$, where $I_{04}=\left\{i_{0}\right\}$. Therefore $\left[h_{0}, f_{\Lambda_{1}}\right]=\left\langle\Lambda_{1}, h_{0}\right\rangle F_{\Lambda_{1}}=-\left\langle M_{1}+\alpha_{i_{0}}, h_{0}\right\rangle F_{\Lambda_{1}}=-F_{\Lambda_{1}}$. We set $e_{1}^{\prime}=e_{i_{0}}, f_{1}^{\prime}=f_{i_{0}}, h_{1}^{\prime}=\alpha_{i_{0}}^{\vee}, e_{2}^{\prime}=2 / 3\left(\operatorname{ad} E_{M_{1}}\right)^{2} e_{1}^{\prime}, f_{2}^{\prime}=\left[F_{\Lambda_{2}}, F_{\Lambda_{1}}\right], h_{2}^{\prime}=2 / 3 h_{0}$. Then we have $\left[h_{1}^{\prime}, e_{2}^{\prime}\right]=-4 e_{2}^{\prime},\left[h_{2}^{\prime}, e_{2}^{\prime}\right]=2 e_{2}^{\prime}, \quad\left[h_{2}^{\prime}, e_{1}^{\prime}\right]=2 / 3 e_{1}^{\prime},\left[e_{i}^{\prime}, f_{j}^{\prime}\right]=\delta_{i j} h_{j}^{\prime}$ $(i=1,2)$. Hence we can reach a contradiction by Lemma 1 .

Lemma 7. (1) Suppose that $\Lambda_{1}+M_{1}=0$. If there is an $s \in I_{1} \backslash\{1\}$ such that $\left[\mathfrak{g}_{-1}^{(1)}, \mathfrak{g}_{1}^{(s)}\right] \neq\{0\}$, then $\Lambda_{1}+M_{s} \neq 0$.

(2) Suppose that $\Lambda_{1}+M_{1} \neq 0$. If there is an $s \in I_{1} \backslash\{1\}$ such that $\left[\mathfrak{g}_{-1}^{(1)}, \mathfrak{g}_{1}^{(s)}\right] \neq$ $\{0\}$, then $\Lambda_{1}+M_{s}=0$.

(3) If $\Lambda_{1}+M_{1} \neq 0$ and there is an $s \in I_{1} \backslash\{1\}$ such that $\left[\mathfrak{g}_{-1}^{(1)}, \mathfrak{g}_{1}^{(s)}\right] \neq\{0\}$ and $\Lambda_{1}+M_{s}=0$, then $h_{1}:=\left[E_{M_{1}}, F_{\Lambda_{1}}\right] \in \mathfrak{z}\left(\mathfrak{g}_{0}\right) \bigoplus \mathfrak{a}_{4}$.

Proof. (1) Now we suppose that there is an $s \in I_{1} \backslash\{1\}$ such that $\left[\mathfrak{g}_{-1}^{(1)}, \mathfrak{g}_{1}^{(s)}\right]$ $\neq\{0\}$ and $\Lambda_{1}+M_{s}=0$. By Lemma 3 , if we set $h_{1}=\left[E_{M_{1}}, F_{\Lambda_{1}}\right]$ and $h_{2}=\left[E_{M_{s}}, F_{\Lambda_{1}}\right]$, then $\left\langle M_{s}, h_{1}\right\rangle \neq 0$ and $\left\langle M_{s}, h_{2}\right\rangle \neq 0$. Here we may assume that $\left\langle M_{s}, h_{1}\right\rangle=$ $\left\langle M_{s}, h_{2}\right\rangle=2$. Replace the $\mathfrak{g}_{0}$-module $\mathfrak{g}_{1}^{(1)}$ by the $\mathfrak{g}_{0}$-module $\widetilde{\mathfrak{g}}_{1}^{(1)}$ with lowest weight $M_{1}$ and the corresponding weight vector $E_{M_{1}}-E_{M_{s}}$. Then $h:=\left[E_{M_{1}}-E_{M_{s}}, F_{\Lambda_{1}}\right]=$ $h_{1}-h_{2}$ and $\left[h, F_{\Lambda_{1}}\right]=0$. By transitivity and Lemma 6 , we have $h \neq 0$, which contradicts Lemma 3.

(2) Suppose that there is an $s \in I_{-1} \backslash\{1\}$ such that $\left[\mathfrak{g}_{-1}^{(1)}, \mathfrak{g}_{1}^{(s)}\right] \neq\{0\}$ and $\Lambda_{1}+$ $M_{s} \neq 0$. By Lemma $4, \Lambda_{1}+M_{1}=\Lambda_{1}+M_{s}=-\theta_{4}$. Then, multiplying, if necessary, $E_{M_{s}}$ by a nonzero constant, we may assume that $\left[E_{M_{1}}, F_{\Lambda_{1}}\right]=\left[E_{M_{s}}, F_{\Lambda_{1}}\right]$, so $\left[E_{M_{1}}-\right.$ $\left.E_{M_{s}}, \mathfrak{g}_{-1}\right]=\{0\}$ by Lemma 6 . By transitivity, we have $E_{M_{1}}=E_{M_{s}}$, which is a contradiction.

(3) It follows immediately from Lemma 3 (2).

Here we remark that under the assumption of Lemma 7 (3) the local Lie algebra $\mathfrak{g}_{-1}^{(1)} \bigoplus\left[\mathfrak{g}_{-1}^{(1)}, \mathfrak{g}_{1}^{(s)}\right] \bigoplus\left(\mathfrak{g}_{1}^{(1)} \bigoplus \mathfrak{g}_{1}^{(s)}\right)$ is isomorphic, as a local Lie algebra, to the local part of $W(n ; \mathbf{1})$ or $K(n ; \mathbf{1} ; 2)$. Moreover $\left[\mathfrak{g}_{-1}^{(1)}, \mathfrak{g}_{1}^{(s)}\right]=\left[\mathfrak{g}_{-1}^{(1)}, \mathfrak{g}_{1}^{(1)}\right] \bigoplus \mathbf{C} h_{1}$ (cf. [Kac68, Lemma 33]).

Lemma 8. Suppose that $\Lambda_{1}+M_{1} \neq 0$ and $\left[\mathfrak{g}_{-1}^{(2)}, \mathfrak{g}_{1}^{(2)}\right] \neq\{0\}$.

(1) If $\Lambda_{2}+M_{2} \neq 0$, then $\mathfrak{g}_{0}^{\prime}$ can be decomposed into a direct sum of semisimple ideals such that $\mathfrak{g}_{0}^{\prime}=\mathfrak{a}^{\prime} \bigoplus \mathfrak{b}^{\prime}, \mathfrak{a}^{\prime}=\operatorname{Ker} \phi_{-1}^{(2)}=\operatorname{Ker} \phi_{1}^{(2)}, \mathfrak{a}_{4} \subset \mathfrak{a}^{\prime}$ and $\mathfrak{b}^{\prime} \subset \mathfrak{a}_{2}$, whence $\left[\left[\mathfrak{g}_{-1}^{(2)}, \mathfrak{g}_{1}^{(2)}\right], \mathfrak{g}_{j}^{(1)}\right]=\{0\}(j=-1,1)$.

(2) If $\Lambda_{2}+M_{2}=0$ and we put $h_{2}=\left[E_{M_{2}}, F_{\Lambda_{2}}\right]$, then $h_{2} \in \mathfrak{z}\left(\mathfrak{g}_{0}\right) \bigoplus \mathfrak{a}_{2}, \mathfrak{a}_{4} \subset$ $\operatorname{Ker} \phi_{-1}^{(2)}$ and $\left\langle\Lambda_{1}, h_{2}\right\rangle=0$, whence $\left[\mathfrak{g}_{-1}^{(2)}, \mathfrak{g}_{1}^{(2)}\right] \subset \mathfrak{z}\left(\mathfrak{g}_{0}\right) \bigoplus \mathfrak{a}_{2}$.

(3) Suppose that $\Lambda_{2}+M_{2}=0$ and there is an $s \in I_{1}$ such that $\left[\mathfrak{g}_{-1}^{(1)}, \mathfrak{g}_{1}^{(s)}\right] \neq$ 
$\{0\}$ and $\Lambda_{1}+M_{s}=0$. If we put $h_{1}=\left[E_{M s}, F_{\Lambda_{1}}\right]$, then $\left\langle\Lambda_{2}, h_{1}\right\rangle=0$, whence $\left[\left[\mathfrak{g}_{-1}^{(1)}, \mathfrak{g}_{1}^{(s)}\right], \mathfrak{g}_{j}^{(2)}\right]=\left[\left[\mathfrak{g}_{-1}^{(2)}, \mathfrak{g}_{1}^{(2)}\right], \mathfrak{g}_{j}^{(s)}\right]=\{0\}(j=-1,1)$.

Proof. (1) By Lemma 4, we can decompose $\mathfrak{g}_{0}^{\prime}$ into a direct sum of semisimple ideals as follows: $\mathfrak{g}_{0}^{\prime}=\mathfrak{a}^{\prime} \bigoplus \mathfrak{b}^{\prime}, \mathfrak{a}^{\prime}=\operatorname{Ker} \phi_{-1}^{(2)}=\operatorname{Ker} \phi_{1}^{(2)}, \mathfrak{b}^{\prime}$ is simple, $\Lambda_{2}+$ $M_{2}=-\theta^{\prime}$, where $\theta^{\prime}$ is the highest root of $\mathfrak{b}^{\prime}$. Now suppose that $\mathfrak{b}^{\prime}=\mathfrak{a}_{4}$ (i.e., $\left.\theta_{4}=\theta^{\prime}\right)$. Then $\left\langle\Lambda_{1}, \theta^{\prime \vee}\right\rangle>0$ and $\left\langle\Lambda_{2}, \theta^{\prime \vee}\right\rangle>0$. Multiplying, if necessary, $F_{\Lambda_{2}}$ by a nonzero number, we may assume that $\left[E_{M_{2}}, F_{\Lambda_{2}}\right]=e_{-\theta^{\prime}}$. Consider the elements $e_{1}^{\prime}=c_{1}\left[E_{M_{1}}, e_{\theta^{\prime}}\right], e_{2}^{\prime}=c_{2}\left[E_{M_{2}}, e_{\theta^{\prime}}\right], f_{1}^{\prime}=F_{\Lambda_{1}}, f_{2}^{\prime}=F_{\Lambda_{2}}, h_{1}^{\prime}=-c_{1} \theta^{\prime \vee}, h_{2}^{\prime}=$ $-c_{2} \theta^{\prime \vee}$, where $c_{i}=2\left\langle\Lambda_{i}, \theta^{\prime \vee}\right\rangle^{-1}(i=1,2)$; these elements satisfy the relation (2.1). Since $c_{i}>0(i=1,2)$, it is impossible by Lemma 1 . Thus $\mathfrak{b}^{\prime} \subset \mathfrak{a}_{2}$ and $\mathfrak{a}_{4} \subset \mathfrak{a}^{\prime}$.

(2) By Lemma $4, \Lambda_{1}+M_{1}=-\theta_{4}$ and $\left\langle\Lambda_{1}, \theta_{4}^{\vee}\right\rangle>0$. By Lemma $3,\left\langle M_{2}, h_{2}\right\rangle \neq$ 0 . Here we may assume that $\left\langle M_{2}, h_{2}\right\rangle=2$. Consider the elements $e_{1}^{\prime}=c\left[E_{M_{1}}, e_{\theta_{4}}\right]$, $e_{2}^{\prime}=E_{M_{2}}, f_{1}^{\prime}=F_{\Lambda_{1}}, f_{2}^{\prime}=F_{\Lambda_{2}}, h_{1}^{\prime}=-c \theta_{4}^{\vee}, h_{2}^{\prime}=h_{2}$, where $c=2\left\langle\Lambda_{1}, \theta_{4}^{\vee}\right\rangle^{-1}$; these elements satisfy the relation (2.1) and correspond to the matrix $\left(\begin{array}{cc}2 & c\left\langle\Lambda_{2}, \theta_{4}^{\vee}\right\rangle \\ -\left\langle\Lambda_{1}, h_{2}\right\rangle & 2\end{array}\right)$. If $\left\langle\Lambda_{2}, \theta_{4}^{\vee}\right\rangle>0$, then it is impossible by Lemma 1 . Thus $\left\langle\Lambda_{2}, \theta_{4}^{\vee}\right\rangle=0$, so $\mathfrak{a}_{4} \subset \operatorname{Ker} \phi_{-1}^{(2)}$. Similarly if $\left\langle\Lambda_{1}, h_{2}\right\rangle \neq 0$, then we can reach a contradiction. By Lemma $3(3), \mathfrak{g}_{0}^{\prime}=\mathfrak{a}_{2}^{\prime} \oplus \mathfrak{a}_{4}^{\prime}$, where $\mathfrak{a}_{2}^{\prime}=\operatorname{Ker} \phi_{-1}^{(2)} \cap \operatorname{Ker} \phi_{1}^{(2)}$. Since $\mathfrak{a}_{4} \subset \mathfrak{a}_{2}^{\prime}$, we have $\mathfrak{a}_{4}^{\prime} \subset \mathfrak{a}_{2}$. Again, by Lemma $3(3)$, we have $h_{2} \in \mathfrak{z}\left(\mathfrak{g}_{0}\right) \bigoplus \mathfrak{a}_{4}^{\prime} \subset$ $\mathfrak{z}\left(\mathfrak{g}_{0}\right) \bigoplus \mathfrak{a}_{2}$.

(3) Suppose that $\left\langle\Lambda_{2}, h_{1}\right\rangle \neq 0$. We set $h_{2}=\left[E_{M_{2}}, F_{\Lambda_{2}}\right]$; then $\left\langle\Lambda_{1}, h_{2}\right\rangle=0$ by (2). Also we may assume that $\left\langle\Lambda_{1}, h_{1}\right\rangle=\left\langle\Lambda_{2}, h_{2}\right\rangle=-2$. Consider the elements $e_{1}^{\prime}=E_{M_{s}}, f_{1}^{\prime}=F_{\Lambda_{1}}, h_{1}^{\prime}=h_{1}, e_{2}^{\prime}=E_{M_{2}}, f_{2}^{\prime}=F_{\Lambda_{2}}, h_{2}^{\prime}=h_{2}$; these elements satisfy the relation (2.1), which contradicts Lemma 1 .

Lemma 9. Suppose that $\Lambda_{1}+M_{1} \neq 0$. Then $\left\langle\Lambda_{s}, \theta_{4}^{\vee}\right\rangle=0$ for all $s \in I_{-1} \backslash\{1\}$, whence $\mathfrak{a}_{4} \subset \operatorname{Ker} \phi_{-1}^{(s)}$.

Proof. Suppose that $\left\langle\Lambda_{s}, \theta_{4}^{\vee}\right\rangle \neq 0$. Applying Lemma 2 to the case when $e_{1}^{\prime}=\left[E_{M_{1}}, e_{\theta_{4}}\right], f_{1}^{\prime}=F_{\Lambda_{1}}, f_{2}^{\prime}=F_{\Lambda_{s}}, h_{1}^{\prime}=-\theta_{4}^{\vee}$, we have a contradiction because $\left\langle\Lambda_{1}, \theta_{4}^{\vee}\right\rangle>0$ and $\left\langle\Lambda_{s}, \theta_{4}^{\vee}\right\rangle>0$.

Here we define subsets $L, M, K, K_{1}, K_{2}, L_{1}, L_{2}, L_{3}, R, R_{1}$ of $I_{-1}$ as follows:

$L=\left\{i \in I_{-1}:\right.$ there is a $j \in I_{1}$ such that $\left[\mathfrak{g}_{-1}^{(i)}, \mathfrak{g}_{1}^{(j)}\right] \neq\{0\}$ and $\left.\Lambda_{i}+M_{j} \neq 0\right\}$,

$M=\left\{i \in I_{-1}:\left[\mathfrak{g}_{-1}^{(i)}, \mathfrak{g}_{1}\right]=\{0\}\right\}$,

$K=I_{-1} \backslash(L \cup M), R=K \cup M$,

$K_{1}=\left\{i \in K: \operatorname{dim} \mathfrak{g}_{-1}^{(i)}=1\right.$ and $\left.\left[\mathfrak{g}_{-1}^{(i)}, \mathfrak{g}_{-1}\right]=\{0\}\right\}$,

$L_{1}=\left\{i \in L:\right.$ there is a $j \in I_{1}$ such that $\left[\mathfrak{g}_{-1}^{(i)}, \mathfrak{g}_{1}^{(j)}\right] \neq\{0\}$ and $\left.\Lambda_{i}+M_{j}=0\right\}$, 


$$
\begin{aligned}
& L_{2}=\left\{i \in L \backslash L_{1}:\left[\mathfrak{g}_{-1}^{(i)}, \mathfrak{g}_{-1}^{(i)}\right]=\{0\}\right\}, \\
& L_{3}=L \backslash\left(L_{1} \cup L_{2}\right), K_{2}=K \backslash K_{1}, R_{1}=R \backslash K_{1} .
\end{aligned}
$$

Then we have $I_{-1}=L_{1} \cup L_{2} \cup L_{3} \cup K \cup M$ (disjoint union).

Also for a subset $Q$ of $I_{-1}$, we define subsets $Q^{\prime}, Q^{\prime \prime}$ and $Q^{\sim}$ of $I_{1}$ as follows: $Q^{\prime}=\left\{j \in I_{1}:\left[\mathfrak{g}_{-1}^{(i)}, \mathfrak{g}_{1}^{(j)}\right] \neq\{0\}, \Lambda_{i}+M_{j}=0\right.$ for some $\left.i \in Q\right\}, Q^{\prime \prime}=\left\{j \in I_{1}:\right.$ $\left[\mathfrak{g}_{-1}^{(i)}, \mathfrak{g}_{1}^{(j)}\right] \neq\{0\}, \Lambda_{i}+M_{j} \neq 0$ for some $\left.i \in Q\right\}, Q^{\sim}=Q^{\prime} \cup Q^{\prime \prime}$. By Lemma 6 , we have $I_{1}=L_{1}^{\prime} \cup L_{1}^{\prime \prime} \cup L_{2}^{\prime \prime} \cup L_{3}^{\prime \prime} \cup K^{\prime}$ (disjoint union). By Lemmas 5 and 7 , we can define a one-to-one correspondence $L_{1} \cup K \ni i \rightarrow i^{\prime} \in\left(L_{1} \cup K\right)^{\prime}$ such that $\left[\mathfrak{g}_{-1}^{(i)}, \mathfrak{g}_{1}^{\left(i^{\prime}\right)}\right] \neq\{0\}$. Similarly we can define a one-to-one correspondence $L \ni i \rightarrow i^{\prime \prime} \in L^{\prime \prime}$ such that $\left[\mathfrak{g}_{-1}^{(i)}, \mathfrak{g}_{1}^{\left(i^{\prime \prime}\right)}\right] \neq\{0\}$.

In the following, for a subset $P$ (resp. $Q$ ) of $I_{-1}$ (resp. $I_{1}$ ), we denote by $\mathfrak{g}_{-1}^{(P)}$ (resp. $\mathfrak{g}_{1}^{(Q)}$ ) the $\mathfrak{g}_{0}$-submodule $\bigoplus_{i \in P} \mathfrak{g}_{-1}^{(i)}$ (resp. $\left.\bigoplus_{i \in Q} \mathfrak{g}_{1}^{(i)}\right)$ of $\mathfrak{g}_{-1}$ (resp. $\mathfrak{g}_{1}$ ). We put $\mathfrak{g}_{-1}^{(\phi)}=\mathfrak{g}_{1}^{(\phi)}=\{0\}$. For a subset $Q$ of $I_{-1}$, we denote by $\mathfrak{t}^{(Q)}$ the graded subalgebra of $\mathfrak{g}$ generated by $\mathfrak{g}_{-1}^{(Q)} \bigoplus\left[\mathfrak{g}_{-1}^{(Q)}, \mathfrak{g}_{1}^{\left(Q^{\prime}\right)}\right] \bigoplus \mathfrak{g}_{1}^{\left(Q^{\prime}\right)}$, and denote by $\mathfrak{m}^{(Q)}$ the graded subalgebra of $\mathfrak{g}$ generated by $\mathfrak{g}_{-1}^{(Q)} \bigoplus\left[\mathfrak{g}_{-1}^{(Q)}, \mathfrak{g}_{1}^{\left(Q^{\sim}\right)}\right] \bigoplus \mathfrak{g}_{1}^{\left(Q^{\Upsilon}\right)}$. Note that $\left[\mathfrak{g}_{-1}^{(Q)}, \mathfrak{g}_{1}^{\left(Q^{\prime \prime}\right)}\right] \subset$ $\left[\mathfrak{g}_{-1}^{(Q)}, \mathfrak{g}_{1}^{\left(Q^{\prime}\right)}\right]$ for $Q \subset L_{1}$. We set $\mathfrak{m}_{p}^{(Q)}=\mathfrak{g}_{p} \cap \mathfrak{m}^{(Q)}, \mathfrak{t}_{p}^{(Q)}=\mathfrak{g}_{p} \cap \mathfrak{t}^{(Q)} ;$ then $\mathfrak{m}^{(Q)}=$ $\bigoplus_{p \in \mathbf{Z}} \mathfrak{m}_{p}^{(Q)}, \mathfrak{t}^{(Q)}=\bigoplus_{p \in \mathbf{Z}} \mathfrak{t}_{p}^{(Q)}$. In particular, when $Q=\{i\}$, we denote by $\mathfrak{m}^{(i)}$ (resp. $\mathfrak{t}^{(i)}$ ) the graded subalgebra $\mathfrak{m}^{(Q)}\left(\right.$ resp. $\left.\mathfrak{t}^{(Q)}\right)$ of $\mathfrak{g}$.

The following lemma plays an important role in the investigation for the structure of $\mathfrak{g}$ in case $L=\phi$.

Lemma 10. Let $\mathfrak{g}=\bigoplus_{p \in \mathbf{Z}} \mathfrak{g}_{p}$ be a GLA of finite depth such that:

(i) $\mathfrak{g}_{-}$is generated by $\mathfrak{g}_{-1}$;

(ii) $\bigoplus_{p \geq 2} \mathfrak{g}_{p}$ contains no ideals of $\mathfrak{g}$;

(iii) $\mathfrak{g}_{\text {loc }}$ is isomorphic, as a local Lie algebra, to a direct product of the local parts of finite dimensional simple GLAs $\mathfrak{s}^{(k)}=\bigoplus_{p \in \mathbf{Z}} \mathfrak{s}_{p}^{(k)}(k \in I)$ such that $\mathfrak{s}_{-1}^{(k)} \neq\{0\}$ for all $k \in I$.

Then:

(1) The subalgebra $\widetilde{\mathfrak{g}}$ of $\mathfrak{g}$ generated by $\mathfrak{g}_{\text {loc }}$ is isomorphic to $\mathfrak{s}:=\prod_{k \in I} \mathfrak{s}^{(k)}$.

(2) If any $\mathfrak{s}^{(k)}$ is not of type $A_{1}$, then $\mathfrak{g}$ is isomorphic to $\mathfrak{s}$.

(3) If $\operatorname{dim} \mathfrak{g}=\infty$, then there is a subset $J$ of I such that $\mathfrak{s}^{(k)}$ is of type $A_{1}$ for $k \in J$ and such that $\mathfrak{g}$ is isomorphic to $\prod_{k \in I \backslash J} \mathfrak{s}^{(k)} \times W(1 ; \mathbf{1})^{J}$ as a GLA, where we denote by $W(1 ; \mathbf{1})^{J}$ the direct product $\prod_{\lambda \in J} \mathfrak{g}_{\lambda}$ when $\mathfrak{g}_{\lambda}=W(1 ; \mathbf{1})$ for all $\lambda \in J$.

Proof. Let $\mathfrak{r}$ be a maximal ideal of $\tilde{\mathfrak{g}}$ contained in $\bigoplus_{p \leq-2} \mathfrak{g}_{p}$. Then $\tilde{\mathfrak{g}} / \mathfrak{r}=$ $\bigoplus_{p \in \mathbf{Z}} \widetilde{\mathfrak{g}}_{p} / \mathfrak{r}_{p}$ is minimal (cf. [Kac68]), so $\tilde{\mathfrak{g}} / \mathfrak{r}$ is isomorphic to $\mathfrak{s}$ as a GLA. In particular, $\operatorname{dim} \mathfrak{g}<\infty$. By Serre's theorem, we have $\mathfrak{s}=\widetilde{\mathfrak{g}}$, so $\mathfrak{g}=\bigoplus_{p \in \mathbf{Z}} \mathfrak{g}_{p}$ is transitive. This proves (1). The prolongation $\operatorname{Prol}(\mathfrak{s}(1))$ of $\mathfrak{s}(1)$ is a direct product of the prolongation Prol $\left(\mathfrak{s}^{(k)}(1)\right)$ of $\mathfrak{s}^{(k)}(1)$. Let $J$ be the subset of $I$ consisting of all in- 
dices $k$ such that $\mathfrak{s}^{(k)}$ is of type $A_{1}$. Then $\operatorname{Prol}\left(\mathfrak{s}^{(k)}(1)\right) \simeq W(1 ; \mathbf{1})$ for $k \in J$ and $\operatorname{Prol}\left(\mathfrak{s}^{(l)}(1)\right)=\mathfrak{s}^{(l)}$ for $l \in I \backslash J$ (cf. [Yat92, Theorem 16]). On the other hand, a subalgebra of $W(1 ; \mathbf{1})$ containing $W(1 ; \mathbf{1})_{l o c}$ is $W(1 ; \mathbf{1})$ or $W(1 ; \mathbf{1})_{l o c}$. This proves (2) and (3).

From Lemma 10, we have

Lemma 11. Let $Q$ be a subset of $K \cup L_{1}$. Then:

(1) $\mathfrak{t}^{(Q)}$ is a finite dimensional semisimple TGLA.

(2) Let $i \in Q$ such that $\left[\mathfrak{g}_{-1}^{(i)}, \mathfrak{g}_{-1}^{(Q \backslash\{i\})}\right]=\{0\}$. Then $\mathfrak{t}^{(Q)}$ is a direct sum of graded ideals $\mathfrak{t}^{(i)}$ and $\mathfrak{t}^{\left(Q_{1}\right)}$, where $Q_{1}=Q \backslash\{i\}$.

Proof. (1) For $i \in Q$, we set $h_{i}=\left[E_{M_{i}{ }^{\prime}}, F_{\Lambda_{i}}\right]$. We may assume that $\left\langle M_{i}, h_{i}\right\rangle=2$. Let $\left\{e_{i}, f_{i}, \alpha_{i}^{\vee}\right\}_{i \in I}$ be the Chevalley basis of the semisimple part of $\left[\mathfrak{g}_{-1}^{(Q)}, \mathfrak{g}_{1}^{\left(Q^{\prime}\right)}\right]$. Consider the elements $e_{i}^{\prime}=E_{M_{i}{ }^{\prime}}, e_{j}^{\prime}=e_{j}, f_{i}^{\prime}=F_{\Lambda_{i}}, f_{j}^{\prime}=f_{j}, h_{i}^{\prime}=$ $h_{i}, h_{j}^{\prime}=\alpha_{j}^{\vee}(i \in Q, j \in I)$; these elements satisfy the relation (2.1). By [Kac68, Theorem 1], the corresponding matrix $\left(a_{i j}\right)$ is one of Cartan matrices of finite dimensional semisimple Lie algebras, so $\mathfrak{t}_{l o c}^{(Q)}$ is a local Lie algebra isomorphic to the local part of a semisimple GLA $\mathfrak{s}=\bigoplus_{p \in \mathbf{Z}} \mathfrak{s}_{p}$. By Lemma 3, $\mathfrak{s}$ is transitive. Our assertion follows from Lemma 10.

(2) There are a simple graded ideal $\mathfrak{a}=\bigoplus_{p \in \mathbf{Z}} \mathfrak{a}_{p}$ and a semisimple graded ideal $\mathfrak{b}=\bigoplus_{p \in \mathbf{Z}} \mathfrak{b}_{p}$ of $\mathfrak{t}^{(Q)}$ such that $\mathfrak{a}_{-1} \supset \mathfrak{g}_{-1}^{(i)}$ and $\mathfrak{t}^{(Q)}=\mathfrak{a} \bigoplus \mathfrak{b}$. Since $\mathfrak{a}_{-1}$ (resp. $\left.\mathfrak{g}_{-1}^{(i)}\right)$ is contragredient to $\mathfrak{a}_{1}$ (resp. $\mathfrak{g}_{1}^{\left(i^{\prime}\right)}$ ) as a $\mathfrak{g}_{0}$-module, $\mathfrak{t}^{(i)}$ is a graded subalgebra of $\mathfrak{a}$. Since $\left[\mathfrak{g}_{-1}^{(i)}, \mathfrak{g}_{-1}^{\left(Q_{1}\right)}\right]=\{0\}, \mathfrak{t}^{(i)}$ is an ideal of $\mathfrak{a}$, so $\mathfrak{t}^{(i)}=\mathfrak{a}$. Since $\mathfrak{g}_{-1}^{(i)}$ is not isomorphic to $\mathfrak{g}_{-1}^{(j)}$ as a $\mathfrak{g}_{0}$-module for $i \neq j$, we have $\mathfrak{t}_{-1}^{(Q)}=\mathfrak{b}_{-1}$. Also since $\mathfrak{b}_{-1}$ (resp. $\mathfrak{t}_{-1}^{\left(Q_{1}\right)}$ ) is contragredient to $\mathfrak{b}_{1}\left(\right.$ resp. $\left.\mathfrak{t}_{1}^{\left(Q_{1}\right)}\right)$ as a $\mathfrak{g}_{0}$-module, we have $\mathfrak{t}_{1}^{\left(Q_{1}\right)}=\mathfrak{b}_{1}$, so $\mathfrak{t}^{\left(Q_{1}\right)}=\mathfrak{b}$. This proves our assertion.

LEMMA 12. We set $\mathfrak{g}_{0}^{\prime \prime}=\left\{x \in \mathfrak{g}_{0}^{\prime}:\left[x, \mathfrak{g}_{1}\right]=\{0\}\right\}$. Then $\mathfrak{g}_{0}^{\prime}=\mathfrak{g}_{0}^{\prime \prime} \bigoplus \mathfrak{b}_{0}$ (where $\mathfrak{b}_{0}$ is the semisimple part of $\left.\left[\mathfrak{g}_{-1}, \mathfrak{g}_{1}\right]\right)$, and $\mathfrak{g}_{0}^{\prime \prime}=\left\{x \in \mathfrak{g}_{0}^{\prime}:\left[x,\left[\mathfrak{g}_{-1}, \mathfrak{g}_{1}\right]\right]=\{0\}\right\}=$ $\left\{x \in \mathfrak{g}_{0}^{\prime}:\left[x, \mathfrak{g}_{-1}^{(H)}\right]=\{0\}\right\}$, where $H=L \cup K$.

Proof. We set $\mathfrak{e}=\left\{x \in \mathfrak{g}_{0}^{\prime}:\left[x,\left[\mathfrak{g}_{-1}, \mathfrak{g}_{1}\right]\right]=\{0\}\right\}$ and $\mathfrak{f}=\left\{x \in \mathfrak{g}_{0}^{\prime}\right.$ : $\left.\left[x, \mathfrak{g}_{-1}^{(H)}\right]=\{0\}\right\}$; then $\mathfrak{g}_{0}^{\prime}=\mathfrak{e} \bigoplus \mathfrak{b}_{0}$. By Lemma $8,\left[\mathfrak{e}, \mathfrak{g}_{-1}^{(L)}\right]=\left[\mathfrak{e}, \mathfrak{g}_{1}^{\left(L^{`}\right)}\right]=\{0\}$. We set $\mathfrak{l}=\mathfrak{m}^{(K)} \bigoplus \mathfrak{e}$ and $\mathfrak{l}_{p}=\mathfrak{l} \cap \mathfrak{g}_{p}$; then $\mathfrak{l}=\bigoplus_{p \in \mathbf{Z}} \mathfrak{l}_{p}$. By Lemma $11, \mathfrak{m}^{(K)}$ is a finite dimensional semisimple GLA. It is easy to prove that $\mathfrak{l}$ is semisimple and $\mathfrak{e}$ is an ideal of $\mathfrak{l}$, so $\left[\mathfrak{e}, \mathfrak{g}_{-1}^{(K)} \bigoplus \mathfrak{g}_{1}^{\left(K^{\prime}\right)}\right]=\{0\}$. Thus $\mathfrak{e} \subset \mathfrak{g}_{0}^{\prime \prime}$ and $\mathfrak{e} \subset \mathfrak{f}$. Conversely since $\mathfrak{g}_{-1}^{(K)}$ is contragredient to $\mathfrak{g}_{1}^{\left(K^{\prime}\right)}$ as a $\mathfrak{g}_{0}$-module, we have $\left[\mathfrak{f}, \mathfrak{g}_{-1}^{\left(K^{\prime}\right)}\right]=\left[\mathfrak{g}_{0}^{\prime \prime}, \mathfrak{g}_{-1}^{(K)}\right]=\{0\}$. By Lemma 4 , [f, $\left.\mathfrak{g}_{1}^{\left(L^{\sim}\right)}\right]=\left[\mathfrak{g}_{0}^{\prime \prime}, \mathfrak{g}_{-1}^{(L)}\right]=\{0\}$, so $\left[\mathfrak{g}_{0}^{\prime \prime}, \mathfrak{g}_{-1}^{(H)}\right]=\left[\mathfrak{f}, \mathfrak{g}_{1}\right]=\{0\}$. Hence $\mathfrak{f} \subset \mathfrak{g}_{0}^{\prime \prime}$ and $\left[\mathfrak{g}_{0}^{\prime \prime},\left[\mathfrak{g}_{-1}, \mathfrak{g}_{1}\right]\right]=\left[\mathfrak{g}_{0}^{\prime \prime},\left[\mathfrak{g}_{-1}^{(H)}, \mathfrak{g}_{1}\right]\right]=\{0\}$, so $\mathfrak{g}_{0}^{\prime \prime}=\mathfrak{e}=\mathfrak{f}$. 
The following lemma is useful for the investigation of the structure of $\mathfrak{g}$ in case $\operatorname{dim} \mathfrak{g}=\infty$.

Lemma 13. Let $\mathfrak{g}=\bigoplus_{p \in \mathbf{Z}} \mathfrak{g}_{p}$ be a TGLA of finite depth satisfying the following condition: $\mathfrak{g}_{-1}=\mathfrak{g}_{-1}^{(1)} \bigoplus \mathfrak{g}_{-1}^{(2)}$, where the $\mathfrak{g}_{-1}^{(i)}$ are irreducible $\mathfrak{g}_{0}$-submodules of $\mathfrak{g}_{-1}$. If $\operatorname{dim} \mathfrak{g}=\infty$, then $\left[\mathfrak{g}_{-1}^{(1)}, \mathfrak{g}_{-1}^{(2)}\right]=\{0\}$.

Proof. We set $V=\left\{x \in \mathfrak{g}_{-1}:\left[x, \mathfrak{g}_{-1}\right]=0\right\}$. Then $V$ is a $\mathfrak{g}_{0}$-submodule of $\mathfrak{g}_{-1}$. Since $\mathfrak{g}_{-1}^{(1)}$ is not isomorphic to $\mathfrak{g}_{-1}^{(2)}$ as a $\mathfrak{g}_{0}$-module (Lemmas 3 and 5 ), $V=V \cap \mathfrak{g}_{-1}^{(1)} \oplus V \cap \mathfrak{g}_{-1}^{(2)}$. Since the $\mathfrak{g}_{0}$-modules $\mathfrak{g}_{-1}^{(i)}(i=1,2)$ are irreducible, if $V \neq\{0\}$, then $\left[\mathfrak{g}_{-1}^{(1)}, \mathfrak{g}_{-1}^{(2)}\right]=\{0\}$. Thus we may assume that $\mathfrak{g}_{-}$is non-degenerate (i.e., $V=\{0\}$ ). By Lemmas 8,9 and 11 , we have $\mathfrak{g}_{0}^{\prime}=\operatorname{Ker} \phi_{-1}^{(1)} \bigoplus \operatorname{Ker} \phi_{-1}^{(2)}$. Since $\mathfrak{g}$ is infinite dimensional, $\mathfrak{d}_{0}(\mathfrak{g}) \neq\{0\}$ and $\mathfrak{d}_{0}(\mathfrak{g})$ is an ideal of $\mathfrak{g}_{0}$. For convenience, we put $\mathfrak{d}_{0}=\mathfrak{d}_{0}(\mathfrak{g})$. Then $\mathfrak{d}_{0}=\left(\mathfrak{d}_{0} \cap \mathfrak{z}\left(\mathfrak{g}_{0}\right)\right) \bigoplus\left(\operatorname{Ker} \phi_{-1}^{(1)} \cap \mathfrak{d}_{0}\right) \bigoplus\left(\operatorname{Ker} \phi_{-1}^{(2)} \cap \mathfrak{d}_{0}\right)$. Now we suppose that $\mathfrak{z}\left(\mathfrak{g}_{0}\right) \cap \mathfrak{d}_{0} \neq\{0\}$. If $\left[\mathfrak{g}_{-1}^{(1)}, \mathfrak{g}_{-1}^{(2)}\right] \neq\{0\}$, then there are $a \in \mathfrak{g}_{-1}^{(1)}$ and $b \in \mathfrak{g}_{-1}^{(2)}$ such that $[a, b] \neq 0$. Let $z \in \mathfrak{z}\left(\mathfrak{g}_{0}\right) \cap \mathfrak{d}_{0} \backslash\{0\}$; then there are complex numbers $c_{i}(i=1,2)$ such that $[z, x]=c_{i} x$ for $x \in \mathfrak{g}_{-1}^{(i)}$. We have $[z,[a, b]]=\left(c_{1}+c_{2}\right)[a, b]=0$, so $c_{1}+c_{2}=$ 0 . In particular, $c_{i} \neq 0(i=1,2)$. For $x, y \in \mathfrak{g}_{-1}^{(i)},[z,[x, y]]=2 c_{i}[x, y]=0$, so $\left[\mathfrak{g}_{-1}^{(i)}, \mathfrak{g}_{-1}^{(i)}\right]=\{0\}(i=1,2)$. Hence $\mathfrak{g}=\bigoplus_{p \in \mathbf{Z}} \mathfrak{g}_{p}$ has a structure of a pseudo-product GLA. By [Tan85, Lemma 1.14], we have $\operatorname{dim} \mathfrak{g}<\infty$, which is a contradiction. Next suppose that $\mathfrak{z}\left(\mathfrak{g}_{0}\right) \cap \mathfrak{d}_{0}=\{0\}$. Then $\left[\left[\mathfrak{g}_{-1}^{(1)}, \mathfrak{d}_{0}\right], \mathfrak{g}_{-1}^{(2)}\right] \subset\left[\left[\mathfrak{g}_{-1}^{(1)}, \mathfrak{d}_{0} \cap \operatorname{Ker} \phi_{-1}^{(2)}\right], \mathfrak{g}_{-1}^{(2)}\right] \subset$ $\left[\mathfrak{g}_{-1}^{(1)},\left[\mathfrak{d}_{0} \cap \operatorname{Ker} \phi_{-1}^{(2)}, \mathfrak{g}_{-1}^{(2)}\right]\right]+\left[\left[\mathfrak{g}_{-1}^{(1)}, \mathfrak{g}_{-1}^{(2)}\right], \mathfrak{d}_{0}\right]=\{0\}$. Similarly we have $\left[\left[\mathfrak{g}_{-1}^{(2)}, \mathfrak{d}_{0}\right], \mathfrak{g}_{-1}^{(1)}\right]=$ $\{0\}$. Since the $\mathfrak{g}_{0}$-module $\mathfrak{g}_{-1}^{(1)}$ is irreducible, if $\left[\mathfrak{g}_{-1}^{(1)}, \mathfrak{d}_{0}\right] \neq\{0\}$, then $\left[\mathfrak{g}_{-1}^{(1)}, \mathfrak{d}_{0}\right]=\mathfrak{g}_{-1}^{(1)}$. Hence $\left[\mathfrak{g}_{-1}^{(1)}, \mathfrak{g}_{-1}^{(2)}\right]=\left[\left[\mathfrak{g}_{-1}^{(1)}, \mathfrak{d}_{0}\right], \mathfrak{g}_{-1}^{(2)}\right]=\{0\}$. Since $\mathfrak{d}_{0} \neq\{0\}$ and the $\mathfrak{g}_{0}$-module $\mathfrak{g}_{-1}$ is faithful, in case $\left[\mathfrak{g}_{-1}^{(1)}, \mathfrak{d}_{0}\right]=\{0\}$, we have $\left[\mathfrak{g}_{-1}^{(2)}, \mathfrak{d}_{0}\right] \neq\{0\}$, so $\left[\mathfrak{g}_{-1}^{(2)}, \mathfrak{d}_{0}\right]=\mathfrak{g}_{-1}^{(2)}$. Hence $\left[\mathfrak{g}_{-1}^{(1)}, \mathfrak{g}_{-1}^{(2)}\right]=\left[\mathfrak{g}_{-1}^{(1)},\left[\mathfrak{g}_{-1}^{(2)}, \mathfrak{d}_{0}\right]\right]=\{0\}$

For a subset $Q$ of $I_{-1}$ such that $M \subset Q$, we set $\widetilde{\mathfrak{m}}^{(Q)}=\mathfrak{m}^{(Q)} \bigoplus \mathfrak{g}_{0}^{\prime \prime}$ and $\widetilde{\mathfrak{m}}_{p}^{(Q)}=\widetilde{\mathfrak{m}}^{(Q)} \cap \mathfrak{g}_{p} ;$ then $\widetilde{\mathfrak{m}}^{(Q)}=\bigoplus_{p \in \mathbf{Z}} \widetilde{\mathfrak{m}}_{p}^{(Q)}$ and $\widetilde{\mathfrak{m}}^{(Q)}$ is a TGLA (Lemmas 6, 11 and 12).

Lemma 14. Suppose that $L=\phi$. Then $\mathfrak{g}^{a}(1)$ is a direct sum of graded ideals $\widetilde{\mathfrak{m}}^{\left(R_{1}\right)}(1)$ and $\mathfrak{m}^{(i)}(1)\left(i \in K_{1}\right)$. Furthermore there are graded ideals $\mathfrak{u}^{(i)}\left(i \in K_{1}\right)$ of Prol $\left(\mathfrak{g}^{a}(1)\right)$ with the following properties:

(i) $\mathfrak{u}^{(i)}(1)=\mathfrak{m}^{(i)}$ and $\mathfrak{u}^{(i)}$ is isomorphic to $W(1 ; \mathbf{1})$;

(ii) $\widetilde{\mathfrak{m}}^{\left(R_{1}\right)}$ is finite dimensional;

(iii) Prol $\left(\mathfrak{g}^{a}(1)\right)$ is a direct sum of graded ideals $\widetilde{\mathfrak{m}}^{\left(R_{1}\right)}$ and $\mathfrak{u}^{(i)}\left(i \in K_{1}\right)$.

Proof. By Lemma $11, \mathfrak{m}^{(K)}$ is a direct sum of graded ideals $\mathfrak{m}^{\left(K_{2}\right)}$ and $\mathfrak{m}^{(i)}$ $\left(i \in K_{1}\right)$. By the definition of $K_{1}, \widetilde{\mathfrak{m}}^{(R)}$ is a direct sum of graded ideals $\widetilde{\mathfrak{m}}^{\left(R_{1}\right)}$ and $\mathfrak{m}^{(i)}\left(i \in K_{1}\right)$. Hence Prol $\left(\mathfrak{g}^{a}(1)\right)$ is a direct sum of graded ideals Prol $\left(\widetilde{\mathfrak{m}}^{\left(R_{1}\right)}(1)\right)$ 
and Prol $\left(\mathfrak{m}^{(i)}(1)\right)\left(i \in K_{1}\right)$. Setting Prol $\left(\mathfrak{m}^{(i)}\right)=\mathfrak{u}^{(i)}\left(i \in K_{1}\right)$, we obtain that $\mathfrak{u}^{(i)}(1)=\mathfrak{m}^{(i)}$ and $\mathfrak{u}^{(i)}$ is isomorphic to $W(1 ; \mathbf{1})$. Suppose that Prol $\left(\mathfrak{m}^{\left(R_{1}\right)}(1)\right)$ is infinite dimensional. For convenience, we put $\mathfrak{e}=\bigoplus_{p \in \mathbf{Z}} \mathfrak{e}_{p}=\operatorname{Prol}\left(\widetilde{\mathfrak{m}}^{\left(R_{1}\right)}(1)\right)$ and $\mathfrak{d}_{p}(\mathfrak{e})=\mathfrak{d}_{p} ;$ then $\mathfrak{d}_{p} \neq\{0\}$ for $p \geq 0,\left[\mathfrak{d}_{p}, \mathfrak{d}_{q}\right] \subset \mathfrak{d}_{p+q}$ and $\left[\mathfrak{d}_{p}, \mathfrak{g}_{-1}\right] \subset \mathfrak{d}_{p-1}$ for $p \geq-1$. Moreover we set $N=\left\{i \in R_{1}:\left[\mathfrak{g}_{-1}^{(i)}, \mathfrak{d}_{1}\right] \neq\{0\}\right\}$. Let $\mathfrak{n}=\bigoplus_{p \in \mathbf{Z}} \mathfrak{n}_{p}$ be the graded subalgebra of $\mathfrak{e}$ generated by $\mathfrak{g}_{-1}^{(N)} \bigoplus\left[\mathfrak{g}_{-1}^{(N)}, \mathfrak{d}_{1}\right] \bigoplus \bigoplus_{p \geq 1} \mathfrak{d}_{p}$. We can easily prove that if $\left[\mathfrak{g}_{-1}^{(i)}, \mathfrak{d}_{1}\right]=\{0\}$, then $\left[\mathfrak{g}_{-1}^{(i)}, \mathfrak{d}_{p}\right]=\{0\}$ for $p \geq 1$. Thus $\mathfrak{n}=\bigoplus_{p \in \mathbf{Z}} \mathfrak{n}_{p}$ is a TGLA. By Lemma $11, \mathfrak{m}^{(N)}$ is a semisimple TGLA, so by Lemma 10 , there are a subset $N_{1}$ of $N$ and graded subalgebras $\mathfrak{u}^{(i)}\left(i \in N_{1}\right)$ of $\mathfrak{n}$ such that: $(1) \mathfrak{u}^{(i)}$ is isomorphic to $W(1 ; \mathbf{1}) ;(2) \mathfrak{m}^{(i)}=\mathfrak{u}^{(i)}(1) ;(3) \mathfrak{n}$ is a direct sum of graded ideals $\widetilde{\mathfrak{m}}^{\left(N_{2}\right)}$ and $\mathfrak{m}^{(i)}\left(i \in N_{1}\right)$, where $N_{2}=N \backslash N_{1}$. Since $\mathfrak{e}$ is infinite dimensional, we have $N_{1} \neq \phi$. Let $i \in N_{1}$ and $j \in I_{-1} \backslash\{i\}$. We decompose $\mathfrak{g}_{0}^{\prime}$ into a direct sum of semisimple ideals as follows: $\mathfrak{g}_{0}^{\prime}=\mathfrak{b}_{1} \oplus \mathfrak{b}_{2}, \mathfrak{b}_{1}=\operatorname{Ker} \phi_{-1}^{(j)}$. Then the graded subalgebra of $\mathfrak{g}$ generated by $\mathfrak{g}_{-1}^{(i)} \bigoplus \mathfrak{g}_{-1}^{(j)} \bigoplus\left(\mathfrak{a}_{0}^{(i)} \bigoplus \mathfrak{b}_{2}\right) \bigoplus \bigoplus_{p \geq 1} \mathfrak{a}_{p}^{(i)}$ is an infinite dimensional TGLA satisfying the assumption of Lemma 13 , so $\left[\mathfrak{g}_{-1}^{(i)}, \mathfrak{g}_{-1}^{(j)}\right]=\{0\}$. Thus $i \in K_{1}$, which is a contradiction. Hence Prol $\left(\widetilde{\mathfrak{m}}^{\left(R_{1}\right)}(1)\right)$ is finite dimensional. Since $\mathfrak{e}_{+}$is generated by $\mathfrak{e}_{1}$, we have $\operatorname{Prol}\left(\widetilde{\mathfrak{m}}^{\left(R_{1}\right)}(1)\right)=\widetilde{\mathfrak{m}}^{\left(R_{1}\right)}$.

Moreover we have

Lemma 15. For $i \in L$ and $j \in I_{-1} \backslash\{i\},\left[\mathfrak{g}_{-1}^{(i)}, \mathfrak{g}_{-1}^{(j)}\right]=\{0\}$.

Proof. We decompose $\mathfrak{g}_{0}^{\prime}$ into a direct sum of semisimple ideals as follows: $\mathfrak{g}_{0}^{\prime}=\left(\operatorname{Ker} \phi_{-1}^{(i)} \cap \operatorname{Ker} \phi_{-1}^{(j)}\right) \bigoplus \mathfrak{b}$. Since $\left[\mathfrak{g}_{-1}^{(s)}, \mathfrak{g}_{1}^{\left(i^{\prime \prime}\right)}\right]=\{0\}$ for all $s \in I_{-1} \backslash\{i\}$ (Lemma 6), the graded subalgebra of $\mathfrak{g}$ generated by $\mathfrak{g}_{-1}^{(i)} \oplus \mathfrak{g}_{-1}^{(j)} \bigoplus \mathfrak{b} \bigoplus \mathfrak{g}_{1}^{\left(i^{\prime \prime}\right)}$ is an infinite dimensional TGLA satisfying the assumption of Lemma 13 , so $\left[\mathfrak{g}_{-1}^{(i)}, \mathfrak{g}_{-1}^{(j)}\right]=\{0\}$.

LEMma 16. For $i \in L_{1}$ and $s \in I_{-1} \backslash\{i\},\left[\left[\mathfrak{g}_{-1}^{(i)}, \mathfrak{g}_{1}^{\left(i^{\prime}\right)}\right], \mathfrak{g}_{-1}^{(s)}\right]=\{0\}$.

Proof. By Lemmas 6 and $15,\left[\mathfrak{g}_{-1}^{(i)}, \mathfrak{g}_{-1}^{(s)}\right]=\left[\mathfrak{g}_{-1}^{(s)}, \mathfrak{g}_{1}^{\left(i^{\prime}\right)}\right]=\{0\}$, so $\left[\left[\mathfrak{g}_{-1}^{(i)}, \mathfrak{g}_{1}^{\left(i^{\prime}\right)}\right]\right.$, $\left.\mathfrak{g}_{-1}^{(s)}\right] \subset\left[\mathfrak{g}_{-1}^{(i)},\left[\mathfrak{g}_{1}^{\left(i^{\prime}\right)}, \mathfrak{g}_{-1}^{(s)}\right]\right]+\left[\mathfrak{g}_{1}^{\left(i^{\prime}\right)},\left[\mathfrak{g}_{-1}^{(i)}, \mathfrak{g}_{-1}^{(s)}\right]\right]=\{0\}$

We summarize the previous results in the following proposition.

Proposition 17. (1) The local part $\mathfrak{g}_{\text {loc }}^{a}$ of $\mathfrak{g}^{a}$ is isomorphic to a direct sum of graded ideals $\mathfrak{m}_{\text {loc }}^{(i)}\left(i \in L \cup K_{1}\right)$ and $\widetilde{\mathfrak{m}}_{\text {loc }}^{\left(R_{1}\right)}$.

(2) $\mathfrak{g}^{a}(1)$ is a direct sum of graded ideals $\mathfrak{m}^{(i)}(1)\left(i \in L_{1} \cup L_{2} \cup K_{1}\right)$ and $\widetilde{\mathfrak{m}}^{(T)}(1)$, where $T=L_{2} \cup R_{1}$.

(3) $\left[\mathfrak{m}_{p}^{(i)}, \widetilde{\mathfrak{m}}_{q}^{\left(Q_{i}\right)}\right]=\{0\}$ for $i \in L_{3}$ and $p, q, p+q \leq 1$, where $Q_{i}=I_{-1} \backslash\{i\}$.

Proof. For $i \in L$, we put $Q_{i}=I_{-1} \backslash\{i\}$. By Lemmas 6, 8, 9 and 12 , $\mathfrak{g}_{l o c}^{a}$ is 
a direct sum of graded ideals $\mathfrak{m}_{\text {loc }}^{(i)}\left(i \in L_{2} \cup L_{3}\right)$ and $\widetilde{\mathfrak{m}}_{\text {loc }}^{(S)}$, where $S=L_{1} \cup R$. By Lemma $15,\left[\mathfrak{g}_{-1}^{(i)}, \mathfrak{g}_{-1}^{\left(Q_{i}\right)}\right]=\{0\}$ for $i \in L$. Therefore, by Lemma $11, \mathfrak{t}^{\left(K \cup L_{1}\right)}$ is a direct sum of graded ideals $\mathfrak{t}^{(i)}\left(i \in L_{1} \cup K_{1}\right)$ and $\mathfrak{t}^{\left(K_{2}\right)}$. Hence by Lemmas 6, 12 and 16, $\widetilde{\mathfrak{m}}_{\text {loc }}^{(S)}$ is a direct sum of graded ideals $\mathfrak{m}_{\text {loc }}^{(i)}\left(i \in L_{1} \cup K_{1}\right)$ and $\widetilde{\mathfrak{m}}_{l o c}^{\left(R_{1}\right)}$. This proves (1). By Lemma 15 and $(1), \mathfrak{m}^{(i)}(1)\left(i \in L_{1} \cup L_{2} \cup K_{1}\right)$ and $\mathfrak{m}^{(T)}(1)$ are graded ideals of $\mathfrak{g}^{a}(1)$. Also for $i \in L_{1}$, there is an element $v_{i}$ of $\mathfrak{z}\left(\mathfrak{m}_{0}^{(i)}\right)$ such that $\left[v_{i}, x\right]=-x$ for $x \in \mathfrak{g}_{-1}^{(i)}$. By Lemma 16, $\left[v_{i}, \mathfrak{g}_{-1}^{\left(Q_{i}\right)}\right]=\{0\}$, so $\widetilde{\mathfrak{m}}_{p}^{\left(Q_{i}\right)} \cap \mathfrak{m}_{p}^{(i)}=\{0\}$ for $i \in L_{1}$ and $p \leq-2$. Thus the assertion (2) follows from (1). The assertion (3) follows from (1), (2) and Lemma 15.

By Lemma 15 and Prop. 17, each $\mathfrak{m}^{(i)}(i \in L)$ is an infinite dimensional irreducible TGLA of finite depth. From well-known facts (cf. [MT70], [KN65], [Kac68]), we have

$$
\left\{\begin{array}{l}
\operatorname{Prol}\left(\mathfrak{m}^{(i)}(1)\right) \simeq W(n ; \mathbf{1})(n \geq 2) \text { or } K(n ; \mathbf{1} ; 2) \text { for } i \in L_{1} \\
\operatorname{Prol}\left(\mathfrak{m}^{(i)}(1)\right) \simeq S(n ; \mathbf{1}) \text { or } H(n ; \mathbf{1} ; 2) \text { for } i \in L_{2} \\
\operatorname{Prol}\left(\mathfrak{m}^{(i)}(1)\right) \simeq \mathfrak{d}(n) \text { for } i \in L_{3} .
\end{array}\right.
$$

Then we have

Proposition 18. Assume that $L_{3}=\phi$. Then there are a subset $K_{3}$ of $K_{1}$ and graded subalgebras $\mathfrak{u}^{(i)}\left(i \in K_{3}\right)$ of $\mathfrak{g}^{a}$ with the following properties:

(1) $\mathfrak{u}^{(i)}$ is isomorphic to $W(1 ; \mathbf{1})$ and $\mathfrak{u}^{(i)}(1)=\mathfrak{m}^{(i)}$;

(2) $\mathfrak{g}^{a}$ is a direct sum of graded ideals $\widetilde{\mathfrak{m}}^{\left(R_{1}\right)}, \mathfrak{u}^{(i)}\left(i \in K_{3}\right)$ and $\mathfrak{m}^{(i)}(i \in L)$, where $R_{2}=R \backslash K_{3}$.

Proof. By Prop. 17, we know that the truncated TGLA $\mathfrak{g}^{a}(1)$ is a direct sum of graded ideals $\mathfrak{m}^{(i)}(1)\left(i \in K_{1} \cup L_{1} \cup L_{2}\right)$ and $\widetilde{\mathfrak{m}}^{\left(R_{1}\right)}(1)$. Here Prol $\left(\mathfrak{g}^{a}(1)\right)$ is a direct sum of graded ideals $\operatorname{Prol}\left(\mathfrak{m}^{(i)}(1)\right)\left(i \in K_{1} \cup L_{1} \cup L_{2}\right)$ and $\operatorname{Prol}\left(\widetilde{\mathfrak{m}}^{\left(R_{1}\right)}(1)\right)$. Since Prol $\left(\mathfrak{m}^{(i)}(1)\right)=\mathfrak{m}^{(i)}(i \in L)$, there is a graded subalgebra $\mathfrak{f}=\bigoplus_{p \in \mathbf{Z}} \mathfrak{f}_{p}$ of Prol $\left(\mathfrak{g}^{a}(1)\right)$ such that $\mathfrak{f}(1)=\widetilde{\mathfrak{m}}^{(R)}(1)$ and such that $\mathfrak{g}^{a}$ is a direct sum of graded ideals $\mathfrak{f}$ and $\mathfrak{m}^{(i)}(i \in L)$. By Lemma 14, there are a subset $K_{3}$ of $K_{1}$ and graded subalgebras $\mathfrak{u}^{(i)}\left(i \in K_{3}\right)$ of $\mathfrak{f}$ such that: $(1) \mathfrak{u}^{(i)}(1)=\mathfrak{m}^{(i)} ;(2) \mathfrak{u}^{(i)}$ is isomorphic to $W(1 ; \mathbf{1}) ;(3) \mathfrak{f}$ is a direct sum of graded ideals $\mathfrak{u}^{(i)}\left(i \in K_{3}\right)$ and $\widetilde{\mathfrak{m}}^{\left(R_{2}\right)}$, where $R_{2}=R \backslash K_{3}$. This proves our assertion.

2.2 The weak envelope of c.r.t.-GLAs of finite depth.

Let $\mathfrak{g}=\bigoplus_{p \in \mathbf{Z}} \mathfrak{g}_{p}$ be a c.r.t.-GLA of finite depth and $\mathcal{G}=\bigoplus_{p \in \mathbf{Z}} \mathcal{G}_{p}$ be the prolongation of $\mathfrak{g}_{-}$. We define the subspace $\mathfrak{g}_{0}^{e}$ of $\mathcal{G}_{0}$ as follows:

$$
\mathfrak{g}_{0}^{e}=\left\{D \in \mathcal{G}_{0}:\left(\operatorname{ad} D \mid \mathfrak{g}_{-1}\right) \circ \varphi=\varphi \circ\left(\operatorname{ad} D \mid \mathfrak{g}_{-1}\right) \text { for all } \varphi \in \mathcal{L}_{\mathfrak{g}_{0}}\left(\mathfrak{g}_{-1}\right)\right\}
$$

Then $\mathfrak{g}_{0}^{e}$ is a subalgebra of $\mathcal{G}_{0}$ such that $\mathfrak{g}_{0} \subset \mathfrak{g}_{0}^{e}$. The following lemma can be easily proved (cf. Ch.8 in [Bou58]). 
Lemma 19. Let $V$ be $a \mathfrak{g}_{0}$-submodule of $\mathfrak{g}_{-1}$. Then:

(1) $V$ is $\mathfrak{g}_{0}^{e}$-stable: $\left[\mathfrak{g}_{0}^{e}, V\right] \subset V$. Consequently $V$ is an irreducible $\mathfrak{g}_{0}$-module if and only if $V$ is an irreducible $\mathfrak{g}_{0}^{e}$-module.

(2) Any $\mathfrak{g}_{0}$-endomorphism of $V$ is also a $\mathfrak{g}_{0}^{e}$-endomorphism of $V$.

Moreover we have

Lemma 20. (1) $\mathfrak{z}\left(\mathfrak{g}_{0}\right) \subset \mathfrak{z}\left(\mathfrak{g}_{0}^{e}\right)$.

(2) $\mathfrak{g}_{p}$ is $\mathfrak{z}\left(\mathfrak{g}_{0}^{e}\right)$-stable for all $p \geq 1$.

Proof. (1) Let $z \in \mathfrak{z}\left(\mathfrak{g}_{0}\right)$. Then ad $z \mid \mathfrak{g}_{-1} \in \mathcal{L}_{\mathfrak{g}_{0}}\left(\mathfrak{g}_{-1}\right)$. By Lemma 19 (2), $\operatorname{ad} z \mid \mathfrak{g}_{-1}$ is a $\mathfrak{g}_{0}^{e}$-endomorphism of $\mathfrak{g}_{-1}$. This means $\left[\left[z, \mathfrak{g}_{0}^{e}\right], \mathfrak{g}_{-1}\right]=\{0\}$. By transitivity, $\left[z, \mathfrak{g}_{0}^{e}\right]=\{0\}$. This proves our assertion.

(2) We use the same notation as in 2.1. Let $z \in \mathfrak{z}\left(\mathfrak{g}_{0}^{e}\right)$. Since $\sum_{i \in L_{3}} \mathfrak{m}_{-2}^{(i)}$ is a $\mathfrak{z}\left(\mathfrak{g}_{0}^{e}\right)$-stable ideal of $\mathfrak{g}$, we may assume that $L_{3}=\phi$. By Prop. 18 , we may assume that $\mathfrak{g}$ is a finite dimensional c.r.t.-GLA or an infinite dimensional irreducible TGLA of finite depth. First we suppose that $\mathfrak{g}$ is a finite dimensional c.r.t.-GLA. Since the $\mathfrak{g}_{0}^{e}$-module $\mathfrak{g}_{-1}^{(i)}$ is irreducible (Lemma $19(1)$ ), there is a complex number $c_{i}$ such that $[z, x]=c_{i} x$ for $x \in \mathfrak{g}_{-1}^{(i)}$. Let $x \in \mathfrak{m}_{-1}^{(i)}$ and $y \in \mathfrak{m}_{1}^{(i)}$. Then $[[z, y], x]=$ $[z,[y, x]]-[y,[z, x]]=-c_{i}[y, x]$, so $\left[[z, y]+c_{i} y, \mathfrak{m}_{-1}^{(i)}\right]=\{0\}$. Since $\left[\mathfrak{m}_{-1}^{(i)}, \mathfrak{m}_{1}^{(j)}\right]=\{0\}$ for $i \neq j$, we have $\left[[z, y]+c_{i} y, \mathfrak{g}_{-1}\right]=\{0\}$. By transitivity, $[z, y]=-c_{i} y$. Thus $\left[\mathfrak{z}\left(\mathfrak{g}_{0}^{e}\right), \mathfrak{g}_{1}\right] \subset \mathfrak{g}_{1}$. Since $\mathfrak{g}_{+}$is generated by $\mathfrak{g}_{1},\left[\mathfrak{z}\left(\mathfrak{g}_{0}^{e}\right), \mathfrak{g}_{+}\right] \subset \mathfrak{g}_{+}$. Next suppose that $\mathfrak{g}=\bigoplus_{p \in \mathbf{Z}} \mathfrak{g}_{p}$ is an infinite dimensional irreducible TGLA of finite depth. Let $x \in \mathfrak{g}_{1}$. As in the first case, $[z, x]=c x$ for some $c \in \mathbf{C}$. Then, by using induction on $p$, we can prove that $\left[z, x_{p}\right]=c p x_{p}$ for $x_{p} \in \mathfrak{g}_{p}$. This proves our assertion.

We define the graded subspace $\mathfrak{g}^{w}=\bigoplus_{p \in \mathbf{Z}} \mathfrak{g}_{p}^{w}$ of $\mathcal{G}=\bigoplus_{p \in \mathbf{Z}} \mathcal{G}_{p}$ as follows:

$$
\mathfrak{g}_{p}^{w}=\mathfrak{g}_{p}(p \neq 0), \quad \mathfrak{g}_{0}^{w}=\mathfrak{g}_{0}+\mathfrak{z}\left(\mathfrak{g}_{0}^{e}\right) .
$$

By Lemmas 19 and $20, \mathfrak{g}^{w}=\bigoplus_{p \in \mathbf{Z}} \mathfrak{g}_{p}^{w}$ becomes a c.r.t.-GLA of finite depth, which is called the weak envelope of $\mathfrak{g}$.

Remark. Let $\mathfrak{g}=\bigoplus_{p \in \mathbf{Z}} \mathfrak{g}_{p}$ be a c.r.t.-GLA of finite depth over $\mathbf{R}$. We define $\mathfrak{g}_{0}^{e}$ as in (2.3). Then $\mathfrak{g}_{0}^{e}$ satisfies the results of Lemmas 19 and 20. Hence we can define the weak envelope $\mathfrak{g}^{w}=\bigoplus_{p \in \mathbf{Z}} \mathfrak{g}_{p}^{w}$ of $\mathfrak{g}$ as in (2.4).

Example. If $\mathfrak{g}=W(n ; \mathbf{1} ; F), C S(n ; \mathbf{1} ; F), C H(n ; \mathbf{1} ; 2 ; F), K(n ; \mathbf{1} ; 2 ; F)$, or $\mathfrak{c} \mathfrak{d}(n ; \mathbf{1} ; F)$, then $\mathfrak{g}^{w}=\mathfrak{g}$. On the other hand, if $\mathfrak{g}=S(n ; \mathbf{1} ; F)(\operatorname{resp} . C H(n ; \mathbf{1} ; 2 ; F)$ or $\mathfrak{d}(n ; F))$, then $\mathfrak{g}^{w}=C S(n ; \mathbf{1} ; F)($ resp. $C H(n ; \mathbf{1} ; 2 ; F)$ or $\mathfrak{c} \mathfrak{d}(n ; F))$.

2.3. Proof of the main theorem.

Theorem 21. Let $\mathfrak{g}=\bigoplus_{p \in \mathbf{Z}} \mathfrak{g}_{p}$ be a c.r.t.-GLA of finite depth over $\mathbf{C}$. Then there are uniquely, up to isomorphism, a c.r.t.-GLA $\mathcal{L}=\bigoplus_{p \in \mathbf{Z}} \mathcal{L}_{p}$ of finite depth and an ideal $\mathfrak{a}$ of $\mathcal{L}^{a}$ with the following properties: 
(1) $\mathcal{L}$ is a direct product of a finite dimensional c.r.t.-GLA $\mathfrak{h}^{(0)}$ and infinite dimensional irreducible TGLAs $\mathfrak{h}^{(i)}(i=1, \ldots, k+l)$ of finite depth such that $\mathfrak{h}^{(i)}(i=1, \ldots, k)\left(\right.$ resp. $\left.\mathfrak{h}^{(i)}(i=k+1, \ldots, k+l)\right)$ are isomorphic to one of $W(n ; \mathbf{1}), C S(n ; \mathbf{1}), C H(n ; \mathbf{1} ; 2), K(n ; \mathbf{1} ; 2)($ resp. $\mathfrak{c o}(n))$;

(2) $\mathfrak{a} \subset \mathfrak{z}\left(\mathcal{L}^{a}\right) \cap \mathcal{L}_{-2}=\mathfrak{z}\left(\left(\mathfrak{h}^{(0)}\right)^{a}\right) \cap \mathcal{L}_{-2} \oplus \sum_{i=k+1}^{k+l} \mathfrak{h}^{(i)} \cap \mathcal{L}_{-2}, \mathfrak{a} \cap \mathfrak{h}^{(i)}=\{0\}$ $(i=0, \ldots, k+l)$ and $\left(\mathfrak{n}_{\mathcal{L}}(\mathfrak{a})\right)^{w}=\mathcal{L}$

(3) $\mathfrak{g}^{w}$ is isomorphic to $\mathfrak{n}_{\mathcal{L}}(\mathfrak{a}) / \mathfrak{a}$.

In particular, if $\mathfrak{z}\left(\mathfrak{g}^{a}\right) \cap \mathfrak{g}_{-2}=\{0\}$, then $\mathfrak{g}^{w}$ is isomorphic to a direct product of a finite dimensional c.r.t.-GLA and infinite dimensional irreducible TGLAs of finite depth isomorphic to one of $W(n ; \mathbf{1}), C S(n ; \mathbf{1}), C H(n ; \mathbf{1} ; 2), K(n ; \mathbf{1} ; 2)$.

Proof. We use the same notation as in 2.1. We set $\mathfrak{m}=\mathfrak{z}\left(\mathfrak{g}^{a}\right) \cap \mathfrak{g}_{-2}$. Then $\mathfrak{m}$ is an ideal of $\mathfrak{g}^{a}$. For $i \in L_{3}, \mathfrak{m}_{-2}^{(i)} \subset \mathfrak{z}\left(\mathfrak{g}^{a}\right) \cap \mathfrak{g}_{-2}$, since the ideal of $\mathfrak{g}^{a}$ generated by $\left[\mathfrak{g}_{-1}^{(i)}, \mathfrak{g}_{-1}^{(i)}\right]$ coincides with itself. Let $\pi: \mathfrak{g}^{a} \rightarrow \mathcal{M}:=\mathfrak{g}^{a} / \mathfrak{m}$ be the natural projection. We put $\mathcal{M}^{(i)}=\pi\left(\mathfrak{m}^{(i)}\right)$ and $\mathcal{M}^{(R)}=\pi\left(\widetilde{\mathfrak{m}}^{(R)}\right)$. By Prop. 18, there are a subset $K_{3}$ of $K_{1}$ and graded subalgebras $\mathfrak{U}^{(i)}\left(i \in K_{3}\right)$ of $\mathcal{M}$ such that: (1) $\mathfrak{U}^{(i)}(1)=\mathcal{M}^{(i)} ;(2) \mathfrak{U}^{(i)}$ is isomorphic to $W(1 ; \mathbf{1}) ;(3) \mathcal{M}$ is a direct sum of ideals $\mathcal{M}^{\left(R_{2}\right)}, \mathfrak{U}^{(i)}\left(i \in K_{3}\right)$ and $\mathcal{M}^{(i)}(i \in L)$, where $R_{2}=R \backslash K_{3}$. We set $\mathfrak{u}^{(i)}=\bigoplus_{p \geq-1} \pi^{-1}\left(\mathfrak{U}_{p}^{(i)}\right)\left(i \in K_{3}\right)$; then $\mathfrak{u}^{(i)}(1)=\mathfrak{m}^{(i)}, \mathfrak{u}^{(i)}$ is isomorphic to $W(1 ; \mathbf{1})$ and $\mathfrak{g}^{a}=\left(\widetilde{\mathfrak{m}}^{\left(R_{2}\right)}+\sum_{i \in L_{3}} \mathfrak{m}^{(i)}\right) \bigoplus \bigoplus_{i \in K_{3}} \mathfrak{u}^{(i)} \bigoplus \bigoplus_{i \in L_{1} \cup L_{2}} \mathfrak{m}^{(i)}$. For convenience, we put $\mathfrak{f}^{(0)}=\widetilde{\mathfrak{m}}^{\left(R_{2}\right)}, \mathfrak{f}^{(i)}=\mathfrak{u}^{(i)}\left(i \in K_{3}\right), \mathfrak{f}^{(i)}=\mathfrak{m}^{(i)}(i \in L)$ and $I=\{0\} \cup K_{3} \cup L$. For $k \in I$, let $\iota_{k}$ be the inclusion map of $\mathfrak{f}^{(k)}$ into $\mathfrak{g}^{a}$. We set $\mathcal{H}=\prod_{i \in I} \mathfrak{f}^{(i)}$. Note that $\mathfrak{z}(\mathcal{H})=\mathfrak{z}\left(\mathfrak{f}^{(0)}\right) \bigoplus \bigoplus_{i \in L_{3}} \mathfrak{f}_{-2}^{(i)}$. Let $\operatorname{pr}_{k}$ be the projection of $\mathcal{H}$ onto $\mathfrak{f}^{(k)}$, and $j_{k}$ be the inclusion map of $\mathfrak{f}^{(k)}$ into $\mathcal{H}$. Then we define a linear mapping $\Phi$ of $\mathcal{H}$ onto $\mathfrak{g}^{a}$ by putting $\Phi(x)=\sum_{k \in I} \iota_{k}\left(\operatorname{pr}_{k}(x)\right)$. Then $\Phi$ is an epimorphism of $\mathcal{H}$ onto $\mathfrak{g}^{a}$ and $\operatorname{Ker} \Phi \subset \mathfrak{z}(\mathcal{H}) \cap \mathcal{H}_{-2}$. Let $\mathcal{K}=\bigoplus_{p \in \mathbf{Z}} \mathcal{K}_{p}$ (resp. $\mathcal{G}=\bigoplus_{p \in \mathbf{Z}} \mathcal{G}_{p}$ ) be the prolongation of $\mathcal{H}_{-}$(resp. $\left.\mathfrak{g}_{-}\right)$. We define a linear mapping $\chi$ of $\mathfrak{g}_{0}^{e}$ into $\mathcal{K}_{0}$ $\left(=\operatorname{Der}_{0}\left(\mathcal{H}_{-}\right)\right)$by putting $[\chi(D), x]=\sum_{k} j_{k}\left(\left[D, \operatorname{pr}_{k}(x)\right]\right)\left(D \in \mathfrak{g}_{0}^{e}, x \in \mathcal{H}_{-}\right)$. Then $\chi$ is a monomorphism of $\mathfrak{g}_{0}^{e}$ into $\mathcal{K}_{0}$ such that $\chi(\Phi(x))=x$ for $x \in \mathcal{H}_{0}$. Since $\Phi[\chi(D), x]=[D, \Phi(x)]$ for $D \in \mathfrak{g}_{0}^{e}$ and $x \in \mathcal{H}_{-}$, we have $[\chi(D), \operatorname{Ker} \Phi] \subset \operatorname{Ker} \Phi$. We define the graded subspace $\mathcal{N}=\bigoplus_{p \in \mathbf{Z}} \mathcal{N}_{p}$ of $\mathcal{K}$ as follows: $\mathcal{N}_{p}=\mathcal{H}_{p}(p \neq 0)$, $\mathcal{N}_{0}=\chi\left(\mathfrak{z}\left(\mathfrak{g}_{0}^{e}\right)\right)+\mathcal{H}_{0}$. Then $\mathcal{N}=\bigoplus_{p \in \mathbf{Z}} \mathcal{N}_{p}$ becomes a c.r.t.-GLA of finite depth. We denote by $\mathcal{L}=\bigoplus_{p \in \mathbf{Z}} \mathcal{L}_{p}$ the weak envelope of $\mathcal{N}$ and we set $\mathfrak{a}=\operatorname{Ker} \Phi$. We define a linear mapping $\Psi$ of $\mathfrak{n}_{\mathcal{L}}(\mathfrak{a})$ into $\mathcal{G}$ as follows: $\Psi \mid \mathcal{L}_{-}=\Phi$ and $[\Psi(D), \Phi(x)]=\Phi[D, x]$ for $x \in \mathcal{L}_{-}$and $D \in \mathcal{L}_{0} \bigoplus \mathcal{L}_{+}$. Then $\Psi$ is an epimorphism of $\mathfrak{n}_{\mathcal{L}}(\mathfrak{a})$ onto $\mathfrak{g}^{w}$ such that $\Psi \mid \mathcal{K}=\Phi$ and $\operatorname{Ker} \Psi=\mathfrak{a}$. Let $v_{i}$ be an element of $\mathcal{K}_{0}$ defined by $\left[v_{i}, x\right]=p \delta_{i j} x$ for $x \in \mathfrak{f}_{p}^{(j)}, p \leq-1, i \in I$. Then $\mathfrak{z}\left(\mathcal{N}_{0}\right)=\mathfrak{z}^{(0)}+\sum_{i \in I} \mathbf{C} v_{i}$, where $\mathfrak{z}^{(0)}=\left\{z \in \mathfrak{z}\left(\mathcal{N}_{0}\right):\left[z, \bigoplus_{i \in L \cup K_{3}} \mathfrak{h}_{-1}^{(i)}\right]=0\right\}$. We set $\mathfrak{h}^{(0)}=\mathfrak{z}^{(0)}+\mathfrak{f}^{(0)}$ and $\mathfrak{h}^{(i)}=\mathfrak{f}^{(i)}+\mathbf{C} v_{i}\left(i \in L \cup K_{3}\right)$. Then $\mathcal{L}$ is a direct sum of graded ideals $\mathfrak{h}^{(i)}(i \in I)$. Moreover, by (2.2), each $\mathfrak{h}^{(i)}\left(i \in L_{1} \cup L_{2}\right)$ (resp. $\left.\mathfrak{h}^{(i)}\left(i \in L_{3}\right)\right)$ is isomorphic to 
one of $W(n ; \mathbf{1}), C S(n ; \mathbf{1}), C H(n ; \mathbf{1} ; 2), K(n ; \mathbf{1} ; 2)$ (resp. $\mathfrak{c d}(n))$. This proves the existence of $\mathcal{L}$ and $\mathfrak{a}$. To prove the uniqueness, we now suppose that there are a c.r.t.-GLA $\mathcal{L}^{\prime}$ and an ideal $\mathfrak{a}^{\prime}$ of $\mathcal{L}^{\prime a}$ such that $\mathcal{L}^{\prime}$ and $\mathfrak{a}^{\prime}$ satisfy the conditions (2) and (3), and $\mathcal{L}^{\prime}$ is a direct product of a finite dimensional c.r.t.-GLA $\mathfrak{h}^{\prime(0)}$ and infinite dimensional irreducible TGLAs $\mathfrak{h}^{\prime(i)}(i=1, \ldots, k+l)$ of finite depth. Let $\Psi^{\prime}$ be the canonical epimorphism of $\mathfrak{n}_{\mathcal{L}^{\prime}}\left(\mathfrak{a}^{\prime}\right)$ onto $\mathfrak{g}^{w}$. Then $\Psi^{\prime}\left(\left(\mathfrak{h}^{\prime(0)}\right)^{a}\right)=\Psi\left(\left(\mathfrak{h}^{(0)}\right)^{a}\right)$ and $\Psi^{\prime}\left(\left(\mathfrak{h}^{\prime(i)}\right)^{a}\right)=\Psi\left(\left(\mathfrak{h}^{(\sigma(i))}\right)^{a}\right)$ for $i=1, \ldots, k+l$ and some permutation $\sigma$ of order $k+l$. Since $\mathfrak{h}^{(i)} \cap \mathfrak{a}=\mathfrak{h}^{(i)} \cap \mathfrak{a}^{\prime}=\{0\}$, there is an isomorphism $\varphi$ of $\mathfrak{n}_{\mathcal{L}^{\prime}}\left(\mathfrak{a}^{\prime}\right)$ onto $\mathfrak{n}_{\mathcal{L}}(\mathfrak{a})$ such that $\Psi^{\prime} \circ \varphi=\Psi$ and $\varphi\left(\mathfrak{a}^{\prime}\right)=\mathfrak{a}$. Finally suppose that $\mathfrak{z}\left(\mathfrak{g}^{a}\right) \cap \mathfrak{g}_{-2}=\{0\}$. Obviously $\Psi\left(\mathfrak{z}\left(\mathcal{L}^{a}\right) \cap \mathcal{L}_{-2}\right) \subset \mathfrak{z}\left(\mathfrak{g}^{a}\right) \cap \mathfrak{g}_{-2}=\{0\}$, so $\mathfrak{z}\left(\mathcal{L}^{a}\right) \cap \mathcal{L}_{-2}=\mathfrak{a}$. On the other hand, $\mathfrak{z}\left(\mathcal{L}^{a}\right) \cap \mathcal{L}_{-2}=\left(\mathfrak{z}\left(\left(\mathfrak{h}^{(0)}\right)^{a}\right) \cap \mathfrak{h}_{-2}^{(0)}\right) \bigoplus \bigoplus_{i \in L_{3}} \mathfrak{h}_{-2}^{(i)}$. Since $\mathfrak{h}^{(i)} \cap \mathfrak{a}=\{0\}(i=0, \ldots, k+l)$, we have $\mathfrak{a}=\mathfrak{z}\left(\mathcal{L}^{a}\right) \cap \mathcal{L}_{-2}=\{0\}$ and $L_{3}=\phi$. Thus $\mathfrak{n}_{\mathcal{L}}(\mathfrak{a})=\mathcal{L}$ and $\operatorname{Ker} \Psi=\{0\}$. This proves our assertion.

EXAMPLE. There are the following typical examples of an infinite dimensional c.r.t.-GLA of finite depth such that $\mathfrak{a}$ is never zero. For a positive integer $m$ and an $l$-tuple $\mathbf{n}=\left(n_{1}, \ldots, n_{l}\right)$ of positive integers such that $\sum_{i=1}^{l} n_{i}=m$, we define a graded subalgebra $\mathfrak{c o}(m ; \mathbf{n} ; F)=\bigoplus_{p \in \mathbf{Z}} \mathfrak{c d}(m ; \mathbf{n} ; F)_{p}$ of $K(m ; \mathbf{1} ; 2 ; F)$ as follows: $\mathfrak{c o}(m ; \mathbf{n} ; F)=\left\{D \in K(m ; \mathbf{1} ; 2 ; F): D \theta_{i} \in F \theta_{i}\right.$ for all $\left.i\right\}$ and $\mathfrak{c o}(m ; \mathbf{n} ; F)_{p}=$ $\mathfrak{c d}(m ; \mathbf{n} ; F) \cap K(m ; \mathbf{1} ; 2 ; F)_{p}$, where $\theta_{i}=\sum_{i=n_{k-1}+1}^{n_{k}} d x_{i} \wedge d x_{i+m}$ and $n_{-1}=0$. Then these GLAs are c.r.t.-GLAs of finite depth. In particular, when $m=2$ and $\mathbf{n}=(1,1)$, this GLA appears in geometry of the Monge-Ampere equation (cf. [Mor79]). Let $\mathcal{L}=\bigoplus_{p \in \mathbf{Z}} \mathcal{L}_{p}=\prod_{i=1}^{l} \mathfrak{c d}\left(n_{i}\right)$ and $u_{i}$ be a basis of $\mathfrak{c} \mathfrak{d}\left(n_{i}\right)_{-2}$. We set $\mathfrak{a}=\sum_{i=1}^{l-1} \mathbf{C}\left(u_{i}-u_{i+1}\right)$. Since $\mathfrak{d}(n)=\left\{D \in K(n): D\left(d \omega_{K}\right)=0\right\}$, we can easily prove that $\mathfrak{a} \subset \mathfrak{z}\left(\mathcal{L}^{a}\right) \cap \mathcal{L}_{-2}$ and $\mathfrak{n}_{\mathcal{L}}(\mathfrak{a}) / \mathfrak{a}$ is isomorphic to $\mathfrak{c} \mathfrak{d}(m ; \mathbf{n} ; F)$.

\section{§3. Real completely reducible transitive graded Lie algebras of fi- nite depth}

In this section, we prove the real version of Theorem 21 .

Let $\mathfrak{g}=\bigoplus_{p \in \mathbf{Z}} \mathfrak{g}_{p}$ be a c.r.t.-GLA of finite depth over $\mathbf{R}$. We decompose the $\mathfrak{g}_{0}$-modules $\mathfrak{g}_{-1}$ and $\mathfrak{g}_{1}$ into a direct sum of irreducible $\mathfrak{g}_{0}$-submodules as follows:

$$
\mathfrak{g}_{-1}=\bigoplus_{i \in I_{-1}} \mathfrak{g}_{-1}^{(i)}, \quad \mathfrak{g}_{1}=\bigoplus_{i \in I_{1}} \mathfrak{g}_{1}^{(i)}
$$

The complexification $\mathfrak{g}_{-1}^{\mathbf{C}}$ (resp. $\left.\mathfrak{g}_{1}^{\mathbf{C}}\right)$ of $\mathfrak{g}_{-1}$ (resp. $\left.\mathfrak{g}_{1}\right)$ is decomposed into a direct sum of irreducible $\mathfrak{g}_{0}^{\mathbf{C}}$-submodules as follows: $\mathfrak{g}_{-1}^{\mathbf{C}}=\bigoplus_{i \in I_{-1,1}} \mathfrak{g}_{-1}^{(i) \mathbf{C}} \bigoplus \bigoplus_{i \in I_{-1,2}}$ $\left(\mathfrak{l}_{-1}^{(i)} \bigoplus \overline{\mathfrak{l}}_{-1}^{(i)}\right), \mathfrak{g}_{1}^{\mathbf{C}}=\bigoplus_{i \in I_{1,1}} \mathfrak{g}_{1}^{(i) \mathbf{C}} \bigoplus \bigoplus_{i \in I_{1,2}}\left(\mathfrak{l}_{1}^{(i)} \bigoplus \overline{\mathfrak{r}}_{1}^{(i)}\right)$, where $\mathfrak{l}_{-1}^{(i)}$ (resp. $\mathfrak{l}_{1}^{(i)}$ ) is an irreducible $\mathfrak{g}_{0}^{\mathbf{C}}$-submodule of $\mathfrak{g}_{-1}^{(i) \mathbf{C}}$ (resp. $\mathfrak{g}_{1}^{(i) \mathbf{C}}$ ).

The following lemma follows from Lemma 6 . 
Lemma 22. (1) Suppose that $\left[\mathfrak{l}_{1}^{(i)}, \mathfrak{g}_{-1}^{\mathbf{C}}\right] \neq\{0\}$ for some $i \in I_{1,2}$. Then there is a unique $j \in I_{-1,2}$ such that $\left[\mathfrak{l}_{1}^{(i)}, \mathfrak{l}_{-1}^{(j)}\right] \neq\{0\}$ or $\left[\mathfrak{l}_{1}^{(i)}, \mathfrak{l}_{-1}^{(j)}\right] \neq\{0\}$. Furthermore, if $\left[\mathfrak{l}_{-1}^{(i)}, \mathfrak{l}_{1}^{(j)}\right] \neq\{0\}$ (resp. $\left.\left[\mathfrak{l}_{-1}^{(i)}, \overline{\mathfrak{l}}_{1}^{(j)}\right] \neq\{0\}\right)$, then $\left[\overline{\mathfrak{l}}_{1}^{(i)}, \overline{\mathfrak{l}}_{-1}^{(j)}\right] \neq\{0\}$ (resp. $\left.\left[\overline{\mathfrak{l}}_{-1}^{(i)}, \mathfrak{l}_{1}^{(j)}\right] \neq\{0\}\right)$. In this case, $\mathfrak{l}_{-1}^{(i)}$ is contragredient to $\mathfrak{l}_{1}^{(i)}$ as a $\mathfrak{g}_{0}^{\mathbf{C}}$-module if and only if $\overline{\mathfrak{l}}_{-1}^{(i)}$ is contragredient to $\overline{\mathfrak{l}}_{1}^{(j)}$ as a $\mathfrak{g}_{0}^{\mathbf{C}}$-module. Also this is equivalent that $\mathfrak{g}_{1}^{(i)}$ is contragredient to $\mathfrak{g}_{1}^{(j)}$ as a $\mathfrak{g}_{0}$-module.

(2) Suppose that $\left[\mathfrak{g}_{-1}^{\mathbf{C}}, \mathfrak{g}_{1}^{(i)}\right] \neq\{0\}$ for some $i \in I_{1,1}$. Then there is a unique $j \in I_{-1,1}$ such that $\left[\mathfrak{g}_{-1}^{(j)}, \mathfrak{g}_{1}^{(i)}\right] \neq\{0\}$.

From this lemma, for $i \in I_{1,2}$, there is a unique $j \in I_{-1,2}$ such that $\left[\mathfrak{l}_{-1}^{(i)}, \mathfrak{l}_{1}^{(j)}\right] \neq$ $\{0\}$ or $\left[\overline{\mathfrak{l}}_{-1}^{(i)}, \mathfrak{l}_{1}^{(j)}\right] \neq\{0\}$. Interchanging, if necessary, to $\mathfrak{l}_{-1}^{(i)}$ and $\overline{\mathfrak{l}}_{-1}^{(i)}$, we may assume that $\left[\mathfrak{l}_{-1}^{(i)}, \mathfrak{l}_{1}^{(j)}\right] \neq\{0\}$. Then $\left[\mathfrak{l}_{-1}^{(i)}, \overline{\mathfrak{l}}_{1}^{(j)}\right] \neq\{0\},\left[\overline{\mathfrak{l}}_{-1}^{(i)}, \mathfrak{l}_{1}^{(j)}\right]=\{0\}$, and $\left[\overline{\mathfrak{l}}_{-1}^{(j)}, \overline{\mathfrak{l}}_{1}^{(i)}\right] \neq\{0\}$.

Here we define subsets $L, L_{1}, L_{2}, L_{3}, K, M$ of $I_{-1}$ as follows:

$$
\begin{aligned}
& L=\left\{i \in I_{-1}: \text { there is an } i^{\prime} \in I_{1} \text { such that }\left[\mathfrak{g}_{-1}^{(i)}, \mathfrak{g}_{1}^{\left(i^{\prime}\right)}\right] \neq\{0\}\right. \text { and } \\
& \left.\mathfrak{g}_{-1}^{(i)} \text { is not contragredient to } \mathfrak{g}_{1}^{\left(i^{\prime}\right)} \text { as a } \mathfrak{g}_{0} \text {-module }\right\}, \\
& M=\left\{i \in I_{-1}:\left[\mathfrak{g}_{-1}^{(i)}, \mathfrak{g}_{1}\right]=\{0\}\right\}, \\
& K=I_{-1} \backslash(L \cup M) \text {, } \\
& L_{1}=\left\{i \in L: \text { there is an } i^{\prime \prime} \in I_{1} \text { such that }\left[\mathfrak{g}_{-1}^{(i)}, \mathfrak{g}_{1}^{\left(i^{\prime \prime}\right)}\right] \neq\{0\}\right. \text { and } \\
& \left.\mathfrak{g}_{-1}^{(i)} \text { is contragredient to } \mathfrak{g}_{1}^{\left(i^{\prime \prime}\right)} \text { as a } \mathfrak{g}_{0} \text {-module }\right\}, \\
& L_{2}=\left\{i \in L:\left[\mathfrak{g}_{-1}^{(i)}, \mathfrak{g}_{-1}^{(i)}\right]=\{0\}\right\}, \\
& L_{3}=L \backslash\left(L_{1} \cup L_{2}\right) \text {. }
\end{aligned}
$$

Then, by Lemmas 5 and 7, we have $I_{-1}=L \cup K \cup M$ and $L=L_{1} \cup L_{2} \cup L_{3}$. Moreover we define subsets $L_{j, i}, K_{i}, M_{i}(i=1,2, j=1,2,3)$ of $I_{-1}$ as follows: $L_{i, j}=L_{j} \cap I_{-1, i}, K_{i}=K \cap I_{-1, i}, M_{i}=M \cap I_{-1, i}$.

For $i \in L_{1}$, we define a correspondence $i \rightarrow\left(i^{\prime}, i^{\prime \prime}\right)$ of $L_{1}$ to $I_{1} \times I_{1}$ as follows: $\left[\mathfrak{g}_{-1}^{(i)}, \mathfrak{g}_{1}^{\left(i^{\prime}\right)}\right] \neq\{0\},\left[\mathfrak{g}_{-1}^{(i)}, \mathfrak{g}^{\left(i^{\prime \prime}\right)}\right] \neq\{0\}, \mathfrak{g}_{-1}^{(i)}$ is contragredient to $\mathfrak{g}_{1}^{\left(i^{\prime}\right)}$ as a $\mathfrak{g}_{0}$-module, and $\mathfrak{g}_{-1}^{(i)}$ is not contragredient to $\mathfrak{g}_{1}^{\left(i^{\prime \prime}\right)}$ as a $\mathfrak{g}_{0}$-module. For $i \in L_{2}$, we define a correspondence $i \rightarrow i^{\prime \prime}$ of $L_{2}$ to $I_{1}$ as follows: $\left[\mathfrak{g}_{-1}^{(i)}, \mathfrak{g}_{1}^{\left(i^{\prime}\right)}\right] \neq\{0\}, \mathfrak{g}_{-1}^{(i)}$ is not contragredient to $\mathfrak{g}_{1}^{\left(i^{\prime \prime}\right)}$ as a $\mathfrak{g}_{0}$-module. Finally we define a correspondence $i \rightarrow i^{\prime}$ of $K$ to $I_{1}$ as follows: $\left[\mathfrak{g}_{-1}^{(i)}, \mathfrak{g}_{1}^{\left(i^{\prime}\right)}\right] \neq\{0\}$. We set subsets $K_{i, j}(i, j=1,2)$ of $I_{1}$ as follows: 


$$
\begin{aligned}
& K_{1,1}=\left\{i \in K_{1}:\right. {\left[\mathfrak{g}_{-1}^{(i)}, \mathfrak{g}_{-1}\right]=\{0\} \text { and } \mathfrak{g}_{-1}^{(i) \mathbf{C}} \bigoplus\left[\mathfrak{g}_{-1}^{(i) \mathbf{C}}, \mathfrak{g}_{1}^{\left(i^{\prime \prime}\right) \mathbf{C}}\right] \bigoplus \mathfrak{g}_{1}^{\left(i^{\prime \prime}\right) \mathbf{C}} } \\
&\text { is a Lie algebra isomorphic to } \mathfrak{s l}(2 ; \mathbf{C})\} \\
& K_{2,1}=\left\{i \in K_{2}:\right. {\left[\mathfrak{l}_{-1}^{(i)}, \mathfrak{g}_{-1}\right]=\{0\} \text { and } \mathfrak{l}_{-1}^{(i)} \bigoplus\left[\mathfrak{l}_{-1}^{(i)}, \mathfrak{l}_{1}^{\left(i^{\prime \prime}\right)}\right] \bigoplus \mathfrak{l}_{1}^{\left(i^{\prime \prime}\right)} } \\
&\text { is a Lie algebra isomorphic to } \mathfrak{s l}(2 ; \mathbf{C})\} \\
& K_{i, 2}=K \backslash K_{i, 1}(i=1,2), \quad K_{i}=K_{1, i} \cup K_{2, i}(i=1,2)
\end{aligned}
$$

We define truncated graded subalgebras $\mathfrak{m}^{(i)}(1)$ and $\mathfrak{m}^{(Q)}(1)$ of $\mathfrak{g}$ as in Section 2 . We set $R=M \cup K \backslash K_{1}$. Then by Prop. $15, \mathfrak{m}^{(i)}(1)$ and $\mathfrak{m}^{(R)}(1)$ are truncated TGLAs. From well-known facts (cf. [MT70], [KN65]), we have

$$
\left\{\begin{array}{l}
\operatorname{Prol}\left(\mathfrak{m}^{(i)}(1)\right) \simeq W(n ; \mathbf{1} ; \mathbf{R})(n \geq 2) \text { or } K(n ; \mathbf{1} ; 2 ; \mathbf{R}) \text { for } i \in L_{1,1} \\
\operatorname{Prol}\left(\mathfrak{m}^{(i)}(1)\right) \simeq S(n ; \mathbf{1} ; \mathbf{R}) \text { or } H(n ; \mathbf{1} ; 2 ; \mathbf{R}) \text { for } i \in L_{1,2} \\
\operatorname{Prol}\left(\mathfrak{m}^{(i)}(1)\right) \simeq \mathfrak{d}(n ; \mathbf{R}) \text { for } i \in L_{1,3} \\
\operatorname{Prol}\left(\mathfrak{m}^{(i)}(1)\right) \simeq W(n ; \mathbf{1})_{\mathbf{R}}(n \geq 2) \text { or } K(n ; \mathbf{1} ; 2)_{\mathbf{R}} \text { for } i \in L_{2,1} \\
\operatorname{Prol}\left(\mathfrak{m}^{(i)}(1)\right) \simeq S(n ; \mathbf{1})_{\mathbf{R}} \text { or } H(n ; \mathbf{1} ; 2)_{\mathbf{R}} \text { for } i \in L_{2,2}^{(2)} \\
\operatorname{Prol}\left(\mathfrak{m}^{(i)}(1)\right) \simeq \mathfrak{d}(n)_{\mathbf{R}} \text { for } i \in L_{2,3} \\
\operatorname{Prol}\left(\mathfrak{m}^{(i)}(1)\right) \simeq W(1 ; \mathbf{1} ; \mathbf{R}) \text { for } i \in K_{1,1} \\
\operatorname{Prol}\left(\mathfrak{m}^{(i)}(1)\right) \simeq W(1 ; \mathbf{1})_{\mathbf{R}} \text { for } i \in K_{2,1}
\end{array}\right.
$$

From these facts, the following theorem can be proved by the similar method to the proof of Theorem 21 .

Theorem 23. Let $\mathfrak{g}=\bigoplus_{p \in \mathbf{Z}} \mathfrak{g}_{p}$ be a c.r.t.-GLA of finite depth over $\mathbf{R}$. Then there are uniquely, up to isomorphism, a c.r.t.-GLA $\mathcal{L}$ of finite depth and an ideal $\mathfrak{a}$ of $\mathcal{L}^{a}$ with the following properties:

(1) $\mathcal{L}$ is a direct product of a finite dimensional c.r.t. $G L A \mathfrak{h}^{(0)}$ and an infinite dimensional irreducible TGLAs $\mathfrak{h}^{(i)}(i=1, \ldots, k+l)$ of finite depth such that $\mathfrak{h}^{(i)}(i=1, \ldots, k)$ (resp. $\left.\mathfrak{h}^{(i)}(i=k+1, \ldots, k+l)\right)$ are isomorphic to one of $W(n ; \mathbf{1} ; \mathbf{R}), C S(n ; \mathbf{1} ; \mathbf{R}), C H(n ; \mathbf{1} ; 2 ; \mathbf{R}), K(n ; \mathbf{1} ; 2 ; \mathbf{R}), W(n ; \mathbf{1})_{\mathbf{R}}, C S(n ; \mathbf{1})_{\mathbf{R}}$, $C H(n ; \mathbf{1} ; 2)_{\mathbf{R}}, K(n ; \mathbf{1} ; 2)_{\mathbf{R}}\left(\right.$ resp. $\left.\mathfrak{c d}(n ; \mathbf{R}), \mathfrak{c d}(n)_{\mathbf{R}}\right) ;$

(2) $\mathfrak{a} \subset \mathfrak{z}\left(\mathcal{L}^{a}\right) \cap \mathcal{L}_{-2}=\mathfrak{z}\left(\left(\mathfrak{h}^{(0)}\right)^{a}\right) \cap \mathcal{L}_{-2} \bigoplus \sum_{i=k+1}^{k+l} \mathfrak{h}^{(i)} \cap \mathcal{L}_{-2}, \mathfrak{a} \cap \mathfrak{h}^{(i)}=$ $\{0\}(i=0, \ldots, k+l)$ and $\left(\mathfrak{n}_{\mathcal{L}}(\mathfrak{a})\right)^{w}=\mathcal{L}$;

(3) $\mathfrak{g}^{w}$ is isomorphic to $\mathfrak{n}_{\mathcal{L}}(\mathfrak{a}) / \mathfrak{a}$.

In particular, if $\mathfrak{z}\left(\mathfrak{g}^{a}\right) \cap \mathfrak{g}_{-2}=\{0\}$, then $\mathfrak{g}^{w}$ is isomorphic to a direct product of a finite dimensional c.r.t.-GLA and infinite dimensional irreducible TGLAs of finite 
depth isomorphic to one of $W(n ; \mathbf{1} ; \mathbf{R}), C S(n ; \mathbf{1} ; \mathbf{R}), C H(n ; \mathbf{1} ; 2 ; \mathbf{R}), K(n ; \mathbf{1} ; 2 ; \mathbf{R})$, $W(n ; \mathbf{1})_{\mathbf{R}}, C S(n ; \mathbf{1})_{\mathbf{R}}, C H(n ; \mathbf{1} ; 2)_{\mathbf{R}}, K(n ; \mathbf{1} ; 2)_{\mathbf{R}}$.

\section{$\S 4$. Structure of non-semisimple pseudo-product GLAs}

In this section, we classify nonsemisimple pseudo-product GLAs $\mathfrak{G}=$ $\left(\mathfrak{g},\left(\mathfrak{g}_{p}\right)_{p \in \mathbf{Z}}, \mathfrak{e}^{1}, \mathfrak{e}^{2}\right)$ of irreducible type such that $\mathfrak{g}_{1} \neq\{0\}$ and $\mathfrak{d}_{-1}(\mathfrak{g}) \neq\{0\}$. Furthermore we investigate the prolongation of $\mathfrak{g}_{-}$, the Lie algebra of all the derivations of $\mathfrak{g}$ and the automorphism group of $\mathfrak{g}$. Also, unless otherwise stated, all the vector spaces considered in this section are assumed to be over $F=\mathbf{C}$ or $\mathbf{R}$.

4.1 The weak envelope of completely reducible pseudo-product GLAs.

Let $\mathfrak{G}=\left(\mathfrak{g},\left(\mathfrak{g}_{p}\right)_{p \in \mathbf{Z}}, \mathfrak{e}^{1}, \mathfrak{e}^{2}\right)$ be a completely reducible pseudo-product GLA and $\mathfrak{g}^{w}=\bigoplus_{p \in \mathbf{Z}} \mathfrak{g}_{p}^{w}$ be the weak envelope of $\mathfrak{g}$. By Lemma 19, the system $\mathfrak{G}^{w}=$ $\left(\mathfrak{g}^{w},\left(\mathfrak{g}_{p}^{w}\right)_{p \in \mathbf{Z}}, \mathfrak{e}^{1}, \mathfrak{e}^{2}\right)$ becomes a completely reducible pseudo-product GLA, which is called the weak envelope of $\mathfrak{G}$. Note that if $\mathfrak{G}$ is of irreducible type, so is $\mathfrak{G}^{w}$.

REMARK. Let $\mathfrak{G}=\left(\mathfrak{g},\left(\mathfrak{g}_{p}\right)_{p \in \mathbf{Z}}, \mathfrak{e}^{1}, \mathfrak{e}^{2}\right)$ be a pseudo-product GLA such that $\mathfrak{g}_{-}$ is non-degenerate and $\mathfrak{g}$ is semisimple, and $\stackrel{\vee}{\mathfrak{G}}=\left(\stackrel{\vee}{\mathfrak{g}},\left(\stackrel{\vee}{\mathfrak{g}_{p}}\right)_{p \in \mathbf{Z}}, \mathfrak{e}^{1}, \mathfrak{e}^{2}\right)$ be the prolongation of $\mathfrak{G}_{\text {. }}$. Then $\mathfrak{g}$ is a finite dimensional c.r.t.-GLA and $\mathfrak{G}=\mathfrak{G}^{w}=\stackrel{\vee}{ }$ (see [Tan85, Lemma 3.1]).

4.2. Let $\mathfrak{g}=\bigoplus_{p \in \mathbf{Z}} \mathfrak{g}_{p}$ be a finite dimensional c.r.t.-GLA. Let $\mathfrak{r}$ be the radical of $\mathfrak{g}$; then $\mathfrak{r}$ is graded, which we write $\mathfrak{r}=\bigoplus_{p \in \mathbf{Z}} \mathfrak{r}_{p}$. There is a graded Levi subalgebra $\mathfrak{s}=\bigoplus_{p \in \mathbf{Z}} \mathfrak{s}_{p}$ of $\mathfrak{g} ;$ further $\mathfrak{r}_{p}=\{0\}$ for $p \geq 1, \mathfrak{r}_{0}=\left\{x \in \mathfrak{z}\left(\mathfrak{g}_{0}\right):\left[x, \mathfrak{g}_{1}\right]=\{0\}\right\}$ and $\mathfrak{r}_{-1}=\mathfrak{b}_{-1}$, where $\mathfrak{b}_{-1}=\left\{x \in \mathfrak{g}_{-1}:\left[x, \mathfrak{g}_{1}\right]=\{0\}\right\}$ (see [Yat88, Prop.2.5]). Note that Prop. 2.5 in [Yat88] is also true in case $F=\mathbf{R}$. We set $\mathfrak{l}=\mathfrak{s} \bigoplus \mathfrak{r}_{0}$.

Lemma 24. Suppose that $\mathfrak{g}$ satisfies the following condition:

(g1) $\mathfrak{g}$ has no non-zero ideal of $\mathfrak{g}$ contained in $\bigoplus_{p \leq-2} \mathfrak{g}_{p}$.

(1) $\left[\mathfrak{r}_{-}, \mathfrak{r}_{-}\right]=\{0\}$.

(2) If $\mathfrak{g}=\bigoplus_{p \in \mathbf{Z}} \mathfrak{g}_{p}$ is depth $\mu \geq 2$, then $\mathfrak{g}_{1} \neq\{0\}$.

Proof. (1) Since $\left[\mathfrak{r}_{-}, \mathfrak{r}_{-}\right]$is an ideal of $\mathfrak{g}$ contained in $\bigoplus_{p \leq-2} \mathfrak{g}_{p}$, it follows from (g1) that $\left[\mathfrak{r}_{-}, \mathfrak{r}_{-}\right]=\{0\}$.

(2) If $\mathfrak{g}_{1}=\{0\}$, then $\bigoplus_{p \leq-2} \mathfrak{g}_{p}$ is a non-zero ideal of $\mathfrak{g}$, which is a contradiction.

LEMMA 25. (1) In order that $\mathfrak{g}$ satisfies (g1), it is sufficient that the following conditions hold:

(g2) $\mathfrak{b}_{-1} \subset \mathfrak{d}_{-1}(\mathfrak{g})$;

(g3) $\left[\mathfrak{b}_{-1}, \mathfrak{b}_{-1}\right]=\{0\}$.

(2) If $\mathfrak{g}_{2}=\{0\}$, then (g1) implies (g2) and (g3). 
Proof. (1) Let $\mathfrak{f}=\bigoplus_{p \in \mathbf{Z}} \mathfrak{f}_{p}$ be a graded ideal of $\mathfrak{g}$ contained in $\bigoplus_{p \leq-2} \mathfrak{g}_{p}$. In particular, $\mathfrak{f}$ is solvable, so $\mathfrak{f} \subset \mathfrak{r}_{-}$. Since the $\mathfrak{l}$-module $\mathfrak{r}_{-}$is completely reducible, there is a graded l-submodule $N=\bigoplus_{p \in \mathbf{Z}} N_{p}$ such that $\mathfrak{r}_{-}=\mathfrak{f} \bigoplus N$. By (g2) and (g3), $\mathfrak{r}_{-}$is generated by $\mathfrak{r}_{-1}$ as an $\mathfrak{l}$-module, so $N=\mathfrak{r}_{-}$and $\mathfrak{f}=\{0\}$. This proves our assertion.

(2) Suppose that $\mathfrak{g}$ satisfies (g1) and $\mathfrak{g}_{2}=\{0\}$. By (g1), $\left[\mathfrak{r}_{-}, \mathfrak{r}_{-}\right]=\{0\}$, so $\left[\mathfrak{b}_{-1}, \mathfrak{b}_{-1}\right]=\{0\}$. Since $\mathfrak{g}_{2}=\{0\}$, we have $\mathfrak{l}_{p}=\{0\}$ for $p \leq-2$, so $\left[\mathfrak{b}_{-1}, \mathfrak{g}_{p}\right]=\{0\}$ for $p \leq-2$. This proves our assertion.

Lemma 26. Suppose that $\mathfrak{b}_{-1} \neq\{0\}$.

(1) If the following condition holds:

(g4) For $x \in \mathfrak{g}_{-1},\left[x, \mathfrak{b}_{-1}\right]=\{0\}$ implies $x \in \mathfrak{b}_{-1}$, then the $\mathfrak{l}$-module $\mathfrak{r}_{-}$is faithful.

(2) If $\mathfrak{g}_{2}=\{0\}$ and $\mathfrak{g}_{-}$is non-degenerate, then $\mathfrak{g}$ satisfies (g4).

Proof. (1) We set $\mathfrak{f}=\left\{x \in \mathfrak{l}:\left[x, \mathfrak{r}_{-}\right]=\{0\}\right\}$. Then $\mathfrak{f}$ is a graded ideal of $\mathfrak{g}$, which we write $\mathfrak{f}=\bigoplus_{p \in \mathbf{Z}} \mathfrak{f}_{p}$. Since $\left[\mathfrak{f}_{-1}, \mathfrak{b}_{-1}\right]=\{0\}, \mathfrak{f}_{-1} \subset \mathfrak{b}_{-1}$ by (g4), so $\mathfrak{f}_{-1}=\{0\}$. By transitivity of $\mathfrak{g}, \mathfrak{f}_{p}=\{0\}$ for $p \geq 0$. If $\mathfrak{f} \neq\{0\}$, then $\mathfrak{f}$ is a solvable ideal of $\mathfrak{g}$. Hence $\mathfrak{f} \subset \mathfrak{r}_{-}$, which is a contradiction. Thus the $\mathfrak{l}$-module $\mathfrak{r}_{-}$is faithful.

(2) Since $\mathfrak{g}_{2}=\{0\}, \mathfrak{l}_{p}=\{0\}$ for $p \leq-2$. Let $x \in \mathfrak{g}_{-1}$ such that $\left[x, \mathfrak{b}_{-1}\right]=$ $\{0\}$. We put $x=x_{l}+x_{b}, x_{l} \in \mathfrak{l}_{-1}$ and $x_{b} \in \mathfrak{b}_{-1}$. Since $\left[\mathfrak{r}_{-}, \mathfrak{r}_{-}\right]$is an $\mathfrak{l}$-submodule of $\mathfrak{r}_{-}$and the $\mathfrak{l}$-module $\mathfrak{r}_{-}$is completely reducible, there is a graded $\mathfrak{l}$-submodule $N=\bigoplus_{p<0} N_{p}$ of $\mathfrak{r}_{-}$such that $\mathfrak{r}_{-}=\left[\mathfrak{r}_{-}, \mathfrak{r}_{-}\right] \bigoplus N$. Then $N_{-1}=\mathfrak{b}_{-1}$, so $\left[\mathfrak{b}_{-1}, \mathfrak{b}_{-1}\right] \cap$ $\left[\mathfrak{l}_{-1}, \mathfrak{b}_{-1}\right]=\{0\}$. Hence $\left[x_{l}, \mathfrak{b}_{-1}\right]=\left[x_{l}, \mathfrak{l}_{-1}\right]=\{0\}$. Since $\mathfrak{g}_{-}$is non-degenerate, $x_{l}=0$ and $x=x_{b}$. This proves our assertion.

Lemma 27. Suppose that $\mathfrak{b}_{-1} \neq\{0\}$. If the conditions (g2) and (g4) hold, then $\left[\mathfrak{l}_{-1}, \mathfrak{l}_{-1}\right]=\{0\}$. Consequently, if the conditions (g2), (g3) and (g4) hold, then the system $\mathfrak{G}=\left(\mathfrak{g},\left(\mathfrak{g}_{p}\right)_{p \in \mathbf{Z}}, \mathfrak{l}_{-1}, \mathfrak{r}_{-1}\right)$ is a pseudo-product $G L A$.

Proof. By (g2), $\mathfrak{r}_{-2}=\left[\mathfrak{b}_{-1}, \mathfrak{b}_{-1}\right]+\left[\mathfrak{l}_{-1}, \mathfrak{b}_{-1}\right]$ and $\mathfrak{r}_{-p}=\left(\operatorname{ad} \mathfrak{l}_{-1}\right)^{p-1} \mathfrak{r}_{-2}$ for $p \geq 3$. Since $\left[\mathfrak{l}_{p}, \mathfrak{b}_{-1}\right]=\{0\}$ for $p \leq-2,\left[\mathfrak{l}_{p}, \mathfrak{r}_{-}\right]=\{0\}$ for $p \leq-2$. By Lemma 26, $\mathfrak{l}_{p}=\{0\}$ for $p \leq-2$, so $\left[\mathfrak{l}_{-1}, \mathfrak{l}_{-1}\right]=\{0\}$. The last assertion is immediate.

In general situation, we have

Proposition 28. Let $\mathfrak{g}=\bigoplus_{p \in \mathbf{Z}} \mathfrak{g}_{p}$ be a c.r.t.-GLA of finite depth. Suppose that $\mathfrak{b}_{-1} \neq\{0\}$. If the conditions (g2), (g3) and (g4) hold, then $\mathfrak{g}=\bigoplus_{p \in \mathbf{Z}} \mathfrak{g}_{p}$ has a structure of a finite dimensional pseudo-product GLA.

Proof. By (g2), (g3) and Lemma 25, g satisfies (g1). Since $\left[\left[\mathfrak{g}_{0}, \mathfrak{z}\left(\mathfrak{g}^{a}\right) \cap\right.\right.$ $\left.\left.\mathfrak{g}_{-2}\right], \mathfrak{g}^{a}\right]=\{0\}, \mathfrak{z}\left(\mathfrak{g}^{a}\right) \cap \mathfrak{g}_{-2}$ is an ideal of $\mathfrak{g}$ contained in $\bigoplus_{p \leq-2} \mathfrak{g}_{p}$. By (g1), $\mathfrak{z}\left(\mathfrak{g}^{a}\right) \cap \mathfrak{g}_{-2}=\{0\}$. Hence by Theorems 21 and 24 , there are a finite dimensional c.r.t.-GLA $\mathfrak{h}^{(0)}$ and infinite dimensional irreducible TGLAs $\mathfrak{h}^{(i)}(i=1, \ldots, l)$ 
fo finite depth such that $\mathfrak{g}^{a}$ is a direct sum of ideals $\mathfrak{h}^{(i)}(i=0, \ldots, l)$. Since $\left[\mathfrak{b}_{-1}, \mathfrak{h}_{-1}^{(i)}\right]=\{0\}(i=1, \ldots, l)$, we have $\mathfrak{g}^{a}=\mathfrak{h}^{(0)}$. Hence $\mathfrak{g}$ is finite dimensional. From Lemma 27, our assertion follows.

4.3. Construction of non-semisimple pseudo-product GLAs.

4.3.1. Let $\mathfrak{l}=\mathfrak{l}_{-1} \oplus \mathfrak{l}_{0} \oplus \mathfrak{l}_{1}$ be a reductive GLA such that $\mathfrak{z}(\mathfrak{l}) \subset \mathfrak{l}_{0}$, and $M=$ $\bigoplus_{p<0} M_{p}$ be a finite dimensional faithful completely reducible graded l-module such that $M$ is generated by $M_{-1}$ as an $\mathfrak{l}$-module. Considering $M$ as a commutative Lie algebra, we define a semi-direct product $\mathfrak{g}=\mathfrak{l} \ltimes M$ of $\mathfrak{l}$ by $M$, and its gradation as follows: $\mathfrak{g}_{p}=M_{p}$ for $p \leq-2, \mathfrak{g}_{-1}=\mathfrak{l}_{-1} \bigoplus M_{-1}, \mathfrak{g}_{p}=\mathfrak{l}_{p}$ for $p \geq 0$. Then we have

Lemma 29. The system $\mathfrak{G}=\left(\mathfrak{g},\left(\mathfrak{g}_{p}\right)_{p \in \mathbf{Z}}, \mathfrak{l}_{-1}, M_{-1}\right)$ is a pseudo-product $G L A$ of depth $\mu \geq 2$ satisfying (g1). Moreover $\mathfrak{G}^{w}=\mathfrak{G}$ if and only if $\operatorname{dim}_{\mathfrak{z}}(\mathfrak{l})=$ $\operatorname{dim} \mathfrak{z}\left(\mathcal{L}_{\mathfrak{l}}(M)\right)$.

PRoof. Since $M$ is generated by $M_{-1}$ as an l-module, $\mathfrak{g}_{-}$is generated by $\mathfrak{g}_{-1}$. For $k \geq 0$, we set $W_{k}=\left\{x \in \mathfrak{g}_{k}:\left[x, \mathfrak{g}_{-}\right]=\{0\}\right\}$ and let $\mathfrak{a}$ be an ideal of $\mathfrak{g}$ generated by $W_{k}$; then $\mathfrak{a} \subset \bigoplus_{p \geq 0} \mathfrak{g}_{p}$. Thus $\mathfrak{a} \subset \mathfrak{l}$ and $[\mathfrak{a}, M]=\{0\}$. Since the $\mathfrak{l}$-module $M$ is faithful, $\mathfrak{a}=\{0\}$, so $\mathfrak{g}=\bigoplus_{p \in \mathbf{Z}} \mathfrak{g}_{p}$ is transitive. Thus $\mathfrak{G}$ is a pseudo-product GLA of depth $\mu \geq 2$. Obviously $\mathfrak{g}=\bigoplus_{p \in \mathbf{Z}}, \mathfrak{g}_{p}$ satisfies (g2) and (g3), so $\mathfrak{g}=\bigoplus_{p \in \mathbf{Z}} \mathfrak{g}_{p}$ satisfies (g1) (Lemma 25). We define a linear mapping $\phi: \mathfrak{z}\left(\mathcal{L}_{\mathfrak{l}}(M)\right) \rightarrow \operatorname{Der}_{0}\left(\mathfrak{g}_{-}\right)$as follows: $\phi(J)(x)=0, \phi(J)(y)=J y$ for $J \in \mathfrak{z}\left(\mathcal{L}_{\mathfrak{l}}(M)\right), x \in \mathfrak{l}_{-1}, y \in M$. From the definition of $\mathfrak{g}^{w}$, we obtain that $\phi$ is an isomorphism of $\mathfrak{z}\left(\mathcal{L}_{\mathfrak{l}}(M)\right)$ onto $\mathfrak{z}(\mathfrak{l})$ if and only if $\mathfrak{G}^{w}=\mathfrak{G}$. This proves our assertion.

We call the system $\mathfrak{G}=\left(\mathfrak{g},\left(\mathfrak{g}_{p}\right)_{p \in \mathbf{Z}}, \mathfrak{l}_{-1}, M_{-1}\right)$ defined above a pseudo-product GLA of type $(\mathfrak{l}, M)$. In particular, if $\widehat{\mathfrak{l}}$ is simple and the $\mathfrak{l}$-module $M$ is irreducible, then $\mathfrak{G}$ is called a pseudo-product GLA of the irreducible type $(\mathfrak{l}, M)$.

Proposition 30. Let $\mathfrak{g}=\bigoplus_{p \in \mathbf{Z}} \mathfrak{g}_{p}$ be a finite dimensional c.r.t.-GLA. Suppose that the condition (g1) holds and $\mathfrak{g}_{-}$is non-degenerate. If $\mathfrak{g}_{2}=\{0\}$, then $\mathfrak{g}=\bigoplus_{p \in \mathbf{Z}} \mathfrak{g}_{p}$ has a structure of a pseudo-product $G L A$ of type $(\mathfrak{l}, M)$.

Proof. We use the same notation as in 4.2. Since $\mathfrak{g}_{2}=\{0\}$, we have $\mathfrak{r}_{-1} \neq$ $\{0\}$ and $\left[\mathfrak{l}_{-1}, \mathfrak{l}_{-1}\right]=\{0\}$. By Lemmas 24 and 26, $\left[\mathfrak{r}_{-}, \mathfrak{r}_{-}\right]=\{0\}$ and the $\mathfrak{l}$-module $\mathfrak{r}_{-}$is faithful. Hence $\mathfrak{g}$ has a structure of a pseudo-product GLA of type $(\mathfrak{l}, M)$.

4.3.2. let $\mathfrak{l}=\mathfrak{l}_{-1} \oplus \mathfrak{l}_{0} \oplus \mathfrak{l}_{1}$ be a reductive GLA such that $\widehat{\mathfrak{l}}$ is simple and $\mathfrak{z}(\mathfrak{l}) \subset \mathfrak{l}_{0}$. Further let $M=\bigoplus_{p=-1}^{-k} M_{p}(k \geq 2)$ be a faithful irreducible graded l-module such that $M_{-1} \neq\{0\}, N$ be an l-submodule of $\Lambda^{2}(M)$ such that the $\mathfrak{l}$-module $\Lambda^{2}(M) / N$ is non-zero and trivial, and $\Phi$ be the projection of $\Lambda^{2}(M)$ onto $\Lambda^{2}(M) / N$. Since the l-module $M$ is irreducible, we can easily prove that $\Phi\left(M_{p}, M_{q}\right)=\{0\}$ for $p+q \neq-k-1$. We set $R=\bigoplus_{p=-1}^{-k-1} R_{p}, R_{p}=M_{p}$ for 
$p \geq-k$ and $R_{-k-1}=\Lambda^{2}(M) / N$. We define the bracket operation on $R$ as follows: $[x, y]=\Phi(x \wedge y)$ for $x, y \in M$ and $\left[R, R_{-k-1}\right]=\{0\}$. Then $R$ becomes a nilpotent GLA such that $[R, R] \subset R_{-k-1}$. Further the $\mathfrak{l}$-module $R$ is graded and $\mathfrak{l}$ is considered as a subalgebra of $\operatorname{Der}(R)$. Thus we can define a semi-direct produt $\mathfrak{g}=\mathfrak{l} \ltimes R$ of $\mathfrak{l}$ by $R$ and its gradation as follows: $\mathfrak{g}_{p}=R_{p}$ for $p \leq-2, \mathfrak{g}_{-1}=\mathfrak{l}_{-1} \bigoplus R_{-1}, \mathfrak{g}_{p}=\mathfrak{l}_{p}$ for $p \geq 0$. Then we have

Lemma 31. The system $\mathfrak{G}=\left(\mathfrak{g},\left(\mathfrak{g}_{p}\right)_{p \in \mathbf{Z}}, \mathfrak{l}_{-1}, R_{-1}\right)$ is a pseudo-product $G L A$ of irreducible type. Moreover $\mathfrak{G}^{w}=\mathfrak{G}$ if and only if $\operatorname{dim}_{\mathfrak{z}}(\mathfrak{l})=\operatorname{dim}_{\mathfrak{z} N}$, where $\mathfrak{z}_{N}=\left\{\varphi \in \mathfrak{z}\left(\mathcal{L}_{\mathfrak{l}}(M)\right): d_{\varphi}^{2}(N) \subset N\right\}$ and $d_{\varphi}^{2}$ is the restriction to $\Lambda^{2}(M)$ of the derivation of $\Lambda(M)$ induced by $\varphi$.

Proof. As in the proof of Lemma 29, we can prove that the system $\mathfrak{G}=$ $\left(\mathfrak{g},\left(\mathfrak{g}_{p}\right)_{p \in \mathbf{Z}}, \mathfrak{l}_{-1}, R_{-1}\right)$ is a pseudo-product GLA. Since $\widehat{\mathfrak{l}}$ is simple and since the $\mathfrak{l}$ module $M$ is irreducible, $\mathfrak{G}$ is of irreducible type. We define a linear mapping $\phi$ of $\mathfrak{z}_{N}$ into $\operatorname{Der}_{0}\left(\mathfrak{g}_{-}\right)$as follows: $\phi(J)(x)=0, \phi(J)(m)=J m, \phi(J)(n)=d_{J}^{2}(n), J \in$ $\mathfrak{z}_{N}, x \in \mathfrak{l}_{-1}, m \in M, n \in R_{-k-1}$, where $d_{J}^{2}$ is the induced endomorphism of $\Lambda^{2}(L) / N$ by $d_{J}^{2}$ such that $\Phi \circ d_{J}^{2}=d_{J}^{2} \circ \Phi$. From the definition of $\mathfrak{g}^{w}$, we obtain that $\phi$ is an isomorphism of $\mathfrak{z}_{N}$ onto $\mathfrak{z}(\mathfrak{l})$ if and only if $\mathfrak{G}^{w}=\mathfrak{G}$. This proves our assertion.

We call the system $\mathfrak{G}=\left(\mathfrak{g},\left(\mathfrak{g}_{p}\right)_{p \in \mathbf{Z}}, \mathfrak{l}_{-1}, R_{-1}\right)$ defined above a pseudo-product GLA of the irreducible type $(\mathfrak{l}, M, N)$ of order $k$. Note that $\mathfrak{d}_{-1}(\mathfrak{g}) \neq\{0\}$ only in the case when $k=2$.

In the case of pseudo-product GLAs of irreducible type, we have

TheOREM 32. Let $\mathfrak{G}=\left(\mathfrak{g},\left(\mathfrak{g}_{p}\right)_{p \in \mathbf{Z}}, \mathfrak{e}^{1}, \mathfrak{e}^{2}\right)$ be a pseudo-product GLA of irreducible type of depth $\mu$. Further assume that $\mathfrak{d}_{-1}(\mathfrak{g}) \neq\{0\}, \mathfrak{g}_{1} \neq\{0\}$ and $\mathfrak{g}_{2}=\{0\}$. Then:

(1) If $\mu \neq 3$ or the condition (g2) holds, then $\mathfrak{G}$ is isomorphic to a pseudoproduct GLA of the irreducible type $(\mathfrak{l}, M)$.

(2) If $\mu=3$ and the condition (g2) does not hold, then $\mathfrak{G}$ is isomorphic to a pseudo-product GLA of the irreducible type $(\mathfrak{l}, M, N)$ of order 2.

Proof. We use the same notation as in 4.2. Since $\mathfrak{g}_{2}=\{0\}, \mathfrak{g}$ is nonsemisimple, so $\mathfrak{b}_{-1} \neq\{0\}$. Also, for convenience, we put $\mathfrak{d}_{-1}=\mathfrak{d}_{-1}(\mathfrak{g})$. Since $\mathfrak{G}$ is of irreducible type, $\mathfrak{g}_{-}$is non-degenerate (cf. the proof of Lemma 13). Since $\mathfrak{e}^{1}$ is not isomorphic to $\mathfrak{e}^{2}$ as a $\mathfrak{g}_{0}$-module ([Yat88, Lemma 2.4]), we have (i) $\mathfrak{b}_{-1}=\mathfrak{e}^{1}, \mathfrak{l}_{-1}=\mathfrak{e}^{2}$ or (ii) $\mathfrak{b}_{-1}=\mathfrak{e}^{2}, \mathfrak{l}_{-1}=\mathfrak{e}^{1}$, so $\left[\mathfrak{b}_{-1}, \mathfrak{b}_{-1}\right]=\left[\mathfrak{l}_{-1}, \mathfrak{l}_{-1}\right]=\{0\}$. If $\mu=2$, then $\mathfrak{d}_{-1}=\mathfrak{g}_{-1}$. If $\mu \geq 3$, then $\mathfrak{d}_{-1}=\mathfrak{b}_{-1}$ or $\mathfrak{d}_{-1}=\mathfrak{l}_{-1}$, since $\mathfrak{d}_{-1}$ is a $\mathfrak{g}_{0}$-submodule of $\mathfrak{g}_{-1}$. Now suppose that the condition (g2) holds. By Lemma 25, the condition (g1) holds, and by the proof of Prop. 30, $\mathfrak{G}$ is a pseudoproduct GLA of type $\left(\mathfrak{l}, \mathfrak{r}_{-}\right)$. Since $\mathfrak{G}$ is of irreducible type, $\widehat{\mathfrak{l}}$ is simple and the 
$\mathfrak{l}$-module $\mathfrak{r}_{-}$is irreducible. Thus $\mathfrak{G}$ is isomorphic to a pseudo-product GLA of the irreducible type $\left(\mathfrak{l}, \mathfrak{r}_{-}\right)$. Next suppose that the condition $(\mathrm{g} 2)$ does not hold. Then $\mathfrak{l}_{-1}=\mathfrak{d}_{-1}$. We set $L=\mathfrak{b}_{-1} \oplus \mathfrak{g}_{-2}$; then $L$ is an $\mathfrak{l}$-submodule of $\mathfrak{r}_{-}$. As in the proof of Lemmas 25 and $26, L$ is a faithful irreducible $l$-module. We set $W=\left\{x \in L:\left[x, \mathfrak{g}_{-3}\right]=\{0\}\right\}$; then $W$ is an l-submodule of $L$, so $W=\{0\}$ or $W=L$. If $W=\{0\}$, then $\left[\mathfrak{d}_{-1}, L\right] \subset W=\{0\}$, which is a contradiction. Therefore $W=L$. Since $\mathfrak{g}_{-4}=\left[\mathfrak{g}_{-1}, \mathfrak{g}_{-3}\right]$, it implies $\mathfrak{g}_{-4}=\{0\}$, so $\mu=3$. Let $\Phi$ be a $\mathfrak{g}_{0}$-epimorphism $\Lambda^{2}(L)$ onto $\mathfrak{g}_{-3}$ defined by $\Phi(x \wedge y)=[x, y](x, y \in L)$, and we put $N=\operatorname{ker} \Phi$. Further let $\Psi$ be the projection of $\Lambda^{2}(L)$ onto $\Lambda^{2}(L) / N$. Then the mapping $\Phi$ induces a $\mathfrak{g}_{0}$-isomorphism $\Phi$ of $\Lambda^{2}(L) / N$ onto $\mathfrak{g}_{-3}$ such that $\Phi=\widetilde{\Phi} \circ \Psi$. Let $\mathfrak{G}^{\prime}=\left(\mathfrak{g}^{\prime}\left(\mathfrak{g}_{p}^{\prime}\right)_{p \in \mathbf{Z}}, \mathfrak{e}^{\prime 1}, \mathfrak{e}^{\prime 2}\right)$ be a pseudo-product GLA of the irreducible type $(\mathfrak{l}, L, N)$. We define a linear mapping $\varphi$ of $\mathfrak{g}^{\prime}$ into $\mathfrak{g}$ as follows: $\varphi|\mathfrak{l} \oplus L=\mathrm{id}, \varphi| \Lambda^{2}(L) / N=\widetilde{\Phi}$. Then $\varphi$ is an isomorphism of $\mathfrak{G}^{\prime}$ onto $\mathfrak{G}$. This proves our assertion.

REMARK. (1) Using the reasoning of the proof of Theorem 30, we can prove the following result: Let $\mathfrak{G}=\left(\mathfrak{g},\left(\mathfrak{g}_{p}\right)_{p \in \mathbf{Z}}, \mathfrak{e}^{1}, \mathfrak{e}^{2}\right)$ be a pseudo-product GLA of irreducible type. Suppose that $\mathfrak{g}$ satisfies the condition "Any ideal in $\mathfrak{g}$ contained in $\bigoplus_{p \leq-2} \mathfrak{g}_{p}$ is contained in $\mathfrak{g}_{-\mu}$ ". If $\mathfrak{g}_{1} \neq\{0\}$ and $\mathfrak{g}_{2}=\{0\}$, then $\mathfrak{G}$ is isomorphic to a pseudo-product GLA of the irreducible type $(\mathfrak{l}, M)$ or of the irreducible type $(\mathfrak{l}, M, N)$ of order $\mu-1$.

(2) Let $\mathfrak{G}=\left(\mathfrak{g},\left(\mathfrak{g}_{p}\right)_{p \in \mathbf{Z}}, \mathfrak{e}^{1}, \mathfrak{e}^{2}\right)$ be a pseudo-product GLA of irreducible type such that $\mathfrak{d}_{-1}(\mathfrak{g}) \neq\{0\}$. If $\mathfrak{g}$ is of depth $\mu \geq 4$, then $\mathfrak{g}_{2}=\{0\}$. Indeed, by [Yat88, Theorems 3.2 and 3.3], if $\mathfrak{g}_{2} \neq\{0\}$, then $\mathfrak{g}$ is simple. However, it is not difficult to show that there is no simple TGLA of depth $\mu \geq 4$ such that $\mathfrak{d}_{-1}(\mathfrak{g}) \neq\{0\}$.

4.4. In this subsection, we parametrize pseudo-product GLAs of the irreducible type $(\mathfrak{l}, M)$ and of the irreducible type $(\mathfrak{l}, M, N)$ of order 2 by a root system of $\mathfrak{l}$ and the highest weight of the $\mathfrak{l}$-module $M$. Also throughout this and next subsection, we assume $F=\mathbf{C}$.

4.4.1. First of all, recall the classification of finite dimensional simple GLAs. Let $\mathfrak{g}=\bigoplus_{p \in \mathbf{Z}} \mathfrak{g}_{p}$ be a finite dimensional simple GLA and $\mathfrak{h}$ be a Cartan subalgebra of $\mathfrak{g}_{0}$. Then $\mathfrak{h}$ is a Cartan subalgebra of $\mathfrak{g}$. Let $\Delta$ be a root system of $(\mathfrak{g}, \mathfrak{h})$. We set $\Delta_{p}=\left\{\alpha \in \Delta: e_{\alpha} \in \mathfrak{g}_{p}\right\}$. Then there are a simple root system $\Pi=\left\{\alpha_{1}, \ldots, \alpha_{l}\right\}$ $(l=$ rank $\mathfrak{g})$ and an $l$-tuple $\mathbf{s}=\left(s_{1}, \ldots, s_{l}\right)$ of nonnegative integers such that $\Delta_{p}=\left\{\alpha=\sum_{i=1}^{l} a_{i} \alpha_{i}: \sum_{i=1}^{l} a_{i} s_{i}=p\right\}$. The $l$-tuple $\mathbf{s}$ of nonnegative integers is determined only by the ordering of $\left(\alpha_{1}, \ldots, \alpha_{l}\right)$. In what follows, we assume that the ordering of $\left(\alpha_{1}, \ldots, \alpha_{l}\right)$ is as in the table of [Bou68]. If $\mathfrak{g}$ is of type $X_{l}$ (X= $A, B, C, D, E, F, G)$, then the GLA $\mathfrak{g}=\bigoplus_{p \in \mathbf{Z}} \mathfrak{g}_{p}$ is said to be of type $\left(X_{l}, \mathbf{s}\right)$. Here we remark that for an automorphism $\tau$ of the Dynkin diagram, a simple GLA of type $\left(X_{l}, \mathbf{s}\right)$ is isomorphic to that of type $\left(X_{l}, \tau(\mathbf{s})\right)$, where $\tau(\mathbf{s})=\left(s_{\tau(1)}, \ldots, s_{\tau(l)}\right)$. We will identify a simple GLA of type $\left(X_{l}, \mathbf{s}\right)$ with that of type $\left(X_{l}, \tau(\mathbf{s})\right)$. 
Let $\mathfrak{a}=\mathfrak{a}_{-1} \bigoplus \mathfrak{a}_{0} \bigoplus \mathfrak{a}_{1}$ be a finite dimensional simple GLA of type $\left(X_{l}, \mathbf{s}\right)$. Since $\mathfrak{a}$ is of depth one, there is an $i_{0}$ such that $s_{i_{0}}=1, s_{j}=0$ for $j \neq i_{0}$. Such a GLA is one of the following types:

$$
\begin{array}{llll}
\left(A_{l}, \varepsilon_{i}\right) \quad(1 \leq i \leq[(l+1) / 2]), & \left(B_{l}, \varepsilon_{1}\right), & \left(C_{l}, \varepsilon_{l}\right), \quad\left(D_{l}, \varepsilon_{1}\right), \\
\left(D_{l}, \varepsilon_{l-1}\right) \quad(l \geq 5), \quad\left(E_{6}, \varepsilon_{1}\right), & \left(E_{7}, \varepsilon_{7}\right) .
\end{array}
$$

Let $\mathfrak{b}$ be a finite dimensional semisimple Lie algebra or $\{0\}$, and $\mathfrak{z}$ be a one dimensional Lie algebra. We set $\mathfrak{l}=\mathfrak{a} \times \mathfrak{b} \times \mathfrak{z}$ and define its gradation as follows: $\mathfrak{l}_{-1}=\mathfrak{a}_{-1}, \mathfrak{l}_{0}=\mathfrak{a}_{0} \times \mathfrak{b} \times \mathfrak{z}, \mathfrak{l}_{1}=\mathfrak{a}_{1}$. Let $\mathfrak{h}$ be a Cartan subalgebra of $\mathfrak{l}$ such that $\mathfrak{a} \cap \mathfrak{h}$ is a Cartan subalgebra of $\mathfrak{a}_{0}$ defining the gradation of $\mathfrak{a}$. Let $E(\lambda)$ $($ resp. $E(\nu+\sigma))$ be a faithful irreducible $\mathfrak{a}$-module (resp. $(\mathfrak{b} \times \mathfrak{z})$-module) with highest weight $\lambda$ (resp. $\nu+\sigma)$, where $\lambda \in(\mathfrak{s} \cap \mathfrak{h})^{*}, \nu \in(\mathfrak{b} \cap \mathfrak{h})^{*}$ and $\sigma \in \mathfrak{z}^{*}$. We set $M=E(\lambda) \otimes E(\nu+\sigma)$. Then $M$ is a faithful irreducible $\mathfrak{l}$-module. Let $\Pi_{1}=\left\{\alpha_{i}\right\}$ be a simple root system of $\mathfrak{a}$ defining the gradation of $\mathfrak{a}$, and $\Pi_{2}=\left\{\beta_{i}\right\}$ be a simple root system of $\mathfrak{b}$. Then $\left\{\alpha_{i}, \beta_{i}, \lambda+\nu+\sigma\right\}$ is a basis of $\mathfrak{h}^{*}$. We define the element $E$ of $\mathfrak{h}$ by $\langle\lambda+\nu+\sigma, E\rangle=-1,\left\langle\alpha_{i_{0}}, E\right\rangle=1,\left\langle\alpha_{j}, E\right\rangle=0$ for $j \neq i_{0},\left\langle\beta_{i}, E\right\rangle=0$ for all $i$. Then $E$ acts semisimply on $M$ and all the eigenvalues are negative integers. We set $M_{p}=\{m \in M: E . m=p m\}$. Then $M=\bigoplus_{p<0} M_{p}$ and it is a graded l-module such that $M_{-1} \neq\{0\}$. We decompose $E$ as follows: $E=E_{1}+E_{2}, E_{1} \in \mathfrak{a}, E_{2} \in \mathfrak{b} \times \mathfrak{z}$. Then $E_{2} \in \mathfrak{z}$. We set $E(\lambda)_{j}=\left\{m \in E(\lambda): E_{1} \cdot m=j m\right\}$. Then $M_{p}=E(\lambda)_{p+\langle\lambda, E\rangle+1} \otimes E(\nu+\sigma)$. Thus the gradation of $M$ does not depend on $\mathfrak{b}$ and $\nu$. Consequently we can construct a pseudo-product GLA $\mathfrak{G}=\left(\mathfrak{g},\left(\mathfrak{g}_{p}\right)_{p \in \mathbf{Z}}, \mathfrak{l}_{-1}, M_{-1}\right)$ of the irreducible type $(\mathfrak{l}, M)$ such that $\mathfrak{G}^{w}=\mathfrak{G}$. Conversely any pseudo-product GLA of the irreducible type $(\mathfrak{l}, M)$ such that $\mathfrak{G}^{w}=\mathfrak{G}$ is described by the above form. Also, we will often denote $\mathfrak{a}_{-1}\left(\right.$ resp. $\left.M_{-1}\right)$ by $\mathfrak{e}^{1}$ (resp. $\left.\mathfrak{e}^{2}\right)$. Furthermore the derived subalgebra $[\mathfrak{g}, \mathfrak{g}]$ of $\mathfrak{g}$ is $\mathfrak{g}_{-} \bigoplus\left(\mathfrak{a}_{0} \bigoplus \mathfrak{b}\right) \bigoplus \mathfrak{g}_{+}$. Note that, for $\sigma^{\prime} \in \mathfrak{z}^{*} \backslash\{0\}$, if we set $M^{\prime}=E(\lambda) \otimes E\left(\nu+\sigma^{\prime}\right)$, then $\mathfrak{G}$ is isomorphic to a pseudo-product GLA of the irreducible type $\left(\mathfrak{l}, M^{\prime}\right)$.

We call the pseudo-product GLA $\mathfrak{G}$ constructed above a pseudo-product GLA of type $\left(X_{l}, \mathbf{s}, \lambda ; \mathfrak{b}, \nu\right)$. Here we remark that, for an automorphism $\tau$ of the Dynkin diagram of $\alpha \times \mathfrak{b}$, a pseudo-product GLA of type $\left(X_{l}, \mathbf{s}, \lambda ; \mathfrak{b}, \nu\right)$ is isomorphic to that of type $\left(X_{l}, \tau(\mathbf{s}), \tau(\lambda) ; \mathfrak{b}, \tau(\nu)\right)$, where $\tau\left(\sum t_{i} \varpi_{i}\right)=\sum t_{i} \varpi_{\tau(i)}$ (where $\left(\varpi_{i}\right)$ is the fundamental weights of $\mathfrak{a} \times \mathfrak{b}$ with respect to $\left.\Pi_{1} \cup \Pi_{2}\right)$. We will identify a pseudoproduct GLA of type $\left(X_{l}, \mathbf{s}, \lambda ; \mathfrak{b}, \nu\right)$ with that of type $\left(X_{l}, \tau(\mathbf{s}), \tau(\lambda) ; \mathfrak{b}, \tau(\nu)\right)$. Also, the depth $\mu$ of $\mathfrak{g}=\bigoplus_{p \in \mathbf{Z}} \mathfrak{g}_{p}$ is given by the following formula: $\mu=\left\langle\lambda-w_{0}(\lambda), \varpi_{i_{0}}^{\vee}\right\rangle+$ 1 , where $\varpi_{i_{0}}^{\vee}$ is the element of $\mathfrak{h} \cap \mathfrak{a}$ defined by $\left\langle\alpha_{j}, \varpi_{i_{0}}^{\vee}\right\rangle=\delta_{j i_{0}}$ and $w_{0}$ is the element of the Weyl group of $\mathfrak{l}$ such that $w_{0}\left(\Pi_{1}\right)=-\Pi_{1}$ and $w_{0}\left(\Pi_{2}\right)=-\Pi_{2}$. For the sake of simplicity, we denote by $\left(X_{l}, \mathbf{s}, \lambda\right)$ the sequence $\left(X_{l}, \mathbf{s}, \lambda ;\{0\}, 0\right)$.

From Theorem 32, we have

Theorem 33. Let $\mathfrak{G}=\left(\mathfrak{g},\left(\mathfrak{g}_{p}\right)_{p \in \mathbf{Z}}, \mathfrak{e}^{1}, \mathfrak{e}^{2}\right)$ be as in Theorem 32 , and let $\stackrel{\vee}{\mathfrak{G}}=$ 
$\left(\mathfrak{g},\left(\mathfrak{g}_{p}\right)_{p \in \mathbf{Z}}, \mathfrak{e}^{1}, \mathfrak{e}^{2}\right)$ be the prolongation of $\mathfrak{G}_{-}$. If $\mu \neq 3$ or the condition (g2) holds, then the weak envelope $\mathfrak{G}^{w}$ of $\mathfrak{G}$ is isomorphic to a pseudo-product GLA of type $\left(X_{l}, \mathbf{s}, \lambda ; \mathfrak{b}, \nu\right)$. In particular, if $\mathfrak{g}_{0}=\mathfrak{\mathfrak { g }}_{0}$, then the pair $(\mathfrak{b}, \nu)$ is $(\{0\}, 0)$ or $\left(A_{n}, \varpi_{1}\right)$, where $n=\operatorname{dim} E(\nu+\sigma)-1$.

Proof. The first assertion follows from Theorem 31 and Lemma 29. Now suppose that $\mathfrak{g}_{0}=\mathfrak{\mathfrak { g }}_{0}$. From the first assertion, $\mathfrak{G}$ is a pseudo-product GLA of type $\left(X_{l}, \mathbf{s}, \lambda ; \mathfrak{b}, \nu\right)$. For $A \in \mathfrak{g l}(E(\nu+\sigma))$, we define $\widetilde{A} \in \operatorname{End}\left(\mathfrak{g}_{-}\right)$as follows: $\widetilde{A} x=0$ for $x \in \mathfrak{e}^{1}, \widetilde{A}\left(m_{1} \otimes m_{2}\right)=m_{1} \otimes A m_{2}$ for $m_{1} \otimes m_{2} \in E(\lambda) \otimes E(\nu+\sigma)$. Then $\widetilde{A} \in \operatorname{Der}_{0}\left(\mathfrak{g}_{-}\right),[\widetilde{A}, \mathfrak{a}]=\{0\}$, and $\widetilde{A}=0$ implies $A=0$. Hence, considering $\mathfrak{g l}(E(\nu+\sigma))$ as a subalgebra of $\mathfrak{g}_{0}$, we have $\mathfrak{g l}(E(\nu+\sigma))=\mathfrak{b} \bigoplus \mathfrak{z}$. Thus $\mathfrak{G}$ is of type $\left(X_{l}, \mathbf{s}, \lambda ; \mathfrak{s l}(E(\nu+\sigma)), \varpi_{1}\right)$ (resp. $\left.\left(X_{l}, \mathbf{s}, \lambda\right)\right)$ in case $\operatorname{dim} E(\nu+\sigma)>1$ (resp. $\operatorname{dim} E(\nu+\sigma)=1)$. This proves our assertion.

4.4.2. Next we consider pseudo-product GLAs of the irreducible type $(\mathfrak{l}, M, N)$ of order 2 . Let $\mathfrak{G}=\left(\mathfrak{g},\left(\mathfrak{g}_{p}\right)_{p \in \mathbf{Z}}, \mathfrak{e}^{1}, \mathfrak{e}^{2}\right)$ be a pseudo-product GLA of irreducible type $(\mathfrak{l}, M, N)$ of order 2 such that $\mathfrak{G}^{w}=\mathfrak{G}$. Then $\mathfrak{l}_{-1}=\mathfrak{d}_{-1}(\mathfrak{g})$ and $\mathfrak{g}_{-3}$ is an ideal of $\mathfrak{g}$. We set $\mathfrak{g}^{\prime}=\mathfrak{g} / \mathfrak{g}_{-3} ;$ then the system $\mathfrak{G}^{\prime}=\left(\mathfrak{g}^{\prime},\left(\mathfrak{g}_{p}^{\prime}\right)_{p \in \mathbf{Z}}, \mathfrak{e}^{1}, \mathfrak{e}^{2}\right)$ is isomorphic to a pseudo-product GLA of depth 2 of type $\left(X_{l}, \mathbf{s}, \lambda ; \mathfrak{b}, \nu\right)$, so $M=$ $E(\lambda) \otimes E(\nu+\sigma)$. Since $2=\left\langle\lambda-w_{0}(\lambda), \varpi_{i_{0}}^{\vee}\right\rangle+1$, the sequence $\left(X_{l}, \mathbf{s}, \lambda\right)$ is one of the following types:

$$
\begin{aligned}
& \left(A_{l}, \varepsilon_{1}, \varpi_{i}\right), \quad\left(A_{l}, \varepsilon_{i}, \varpi_{1}\right), \quad\left(B_{l}, \varepsilon_{1}, \varpi_{l}\right), \quad\left(C_{l}, \varepsilon_{l}, \varpi_{1}\right), \\
& \left(D_{l}, \varepsilon_{1}, \varpi_{l-1}\right) \quad(l \geq 4), \quad\left(D_{l}, \varepsilon_{l-1}, \varpi_{1}\right) \quad(l \geq 5) .
\end{aligned}
$$

We set $S_{\lambda}=S^{2}(E(\lambda)), S_{\nu}=S^{2}(E(\nu+\sigma)), A_{\lambda}=\Lambda^{2}(E(\lambda)), A_{\nu}=\Lambda^{2}(E(\nu+\sigma))$. Then we can identify $\Lambda^{2}(M)$ with $\left(S_{\lambda} \otimes A_{\nu}\right) \bigoplus\left(A_{\lambda} \otimes S_{\nu}\right)$. We denote by $l_{s}$ (resp. $l_{a}$ ) the multiplicity of a trivial irreducible $\widehat{\mathfrak{l}}$-module in $S_{\lambda}$ (resp. $A_{\lambda}$ ). Since the $\widehat{\mathfrak{l}}$ module $\Lambda^{2}(M) / N$ is trivial, $l_{s}+l_{a} \geq 1$. By [Bou75, Ch.VIII, $\S 7$, Prop.12], $l_{s}+l_{a}=1$ and $w_{0}(\lambda)=-\lambda$. Hence by $(4.1)$ the sequence $\left(X_{l}, \mathbf{s}, \lambda\right)$ is one of the following types:

$$
\begin{aligned}
& \left(A_{2 l-1}, \varepsilon_{1}, \varpi_{l}\right), \quad\left(B_{l}, \varepsilon_{1}, \varpi_{l}\right), \quad\left(C_{l}, \varepsilon_{l}, \varpi_{1}\right), \\
& \left(D_{l}, \varepsilon_{1}, \varpi_{l-1}\right) \quad(l \geq 4, l=\text { even }), \quad\left(D_{l}, \varepsilon_{l-1}, \varpi_{1}\right) \quad(l \geq 5) .
\end{aligned}
$$

If $l_{s}=1$ (resp. $l_{a}=1$ ), then there are a trivial irreducible $\widehat{\mathfrak{l}}$-submodule $L_{1}$ of $S_{\lambda}$ (resp. $\left.A_{\lambda}\right)$ and a $(\mathfrak{b} \times \mathfrak{z})$-submodule $L_{2}$ of $A_{\nu}\left(\right.$ resp. $\left.S_{\nu}\right)$ such that $\Lambda^{2}(M) / N$ is isomorphic to $L_{1} \otimes L_{2}$ as an l-module. We denote by $\Psi$ the isomorphism of $L_{1} \otimes L_{2}$ onto $\Lambda^{2}(M) / N$. Let $\phi$ be the projection of $\Lambda^{2}(M)$ onto $\Lambda^{2}(M) / N$. Then Ker $\phi \supset$ $A_{\lambda} \otimes S_{\nu}$ (resp. $S_{\lambda} \otimes A_{\nu}$ ) and there are an $\widehat{l}$-homomorphism $\varphi$ of $S_{\lambda}$ (resp. $A_{\lambda}$ ) onto $L_{1}$ and a $(\mathfrak{b} \times \mathfrak{z})$-homomorphism $\psi$ of $A_{\nu}\left(\right.$ resp. $\left.S_{\nu}\right)$ onto $L_{2}$ such that $\Phi \mid S_{\lambda} \otimes A_{\nu}=$ $\Psi \circ(\varphi \otimes \psi)\left(\right.$ resp. $\left.\Phi \mid A_{\lambda} \otimes S_{\nu}=\Psi \circ(\varphi \otimes \psi)\right)$ and $\operatorname{Ker}(\varphi \otimes \psi) \bigoplus\left(A_{\lambda} \otimes S_{\nu}\right)=N$ 
(resp. $\left.\operatorname{Ker}(\varphi \otimes \psi) \bigoplus\left(S_{\lambda} \otimes A_{\nu}\right)=N\right)$. Note that $\varphi$ is uniquely, up to a constant factor, determined from $N$. Conversely, given a pseudo-product GLA of depth 2 of type $\left(X_{l}, \mathbf{s}, \lambda ; \mathfrak{b}, \nu\right), L_{2}$ and $\psi$ satisfying the above conditions, we can construct a pseudoproduct GLA of the irreducible type $(\mathfrak{l}, M, N)$ of order 2 . We call the pseudo-product GLA $\mathfrak{G}$ constructed above a pseudo-product GLA of type $\left(X_{l}, \mathbf{s}, \lambda ; \mathfrak{b}, \nu ; L_{2}, \psi\right)$. If $\Lambda^{2}(M) / N$ is an irreducible $\mathfrak{l}$-module and if the multiplicity of an irreducible $\mathfrak{l}$ submodule of $\Lambda^{2}(M)$ isomorphic to $\Lambda^{2}(M) / N$ is one, then $L_{2}$ (resp. $\psi$ ) is uniquely (resp. uniquely up to a constant factor) determined from $\Lambda^{2}(M) / N$. In this case, if $\Lambda^{2}(M) / N$ is an irreducible $\mathfrak{b}$-module with lowest weight $\omega$, then we denote by $\left(X_{l}, \mathbf{s}, \lambda ; \mathfrak{b}, \nu ; \omega\right)$ the sequence $\left(X_{l}, \mathbf{s}, \lambda ; \mathfrak{b}, \nu ; L_{2}, \psi\right)$. For the sake of simplicity, we denote by $\left(X_{l}, \mathbf{s}, \lambda ; 0\right)$ the sequence $\left(X_{l}, \mathbf{s}, \lambda ;\{0\}, 0 ; 0\right)$. Then the following theorem follows imediately from Theorem 32 .

Theorem 34. Let $\mathfrak{G}=\left(\mathfrak{g},\left(\mathfrak{g}_{p}\right)_{p \in \mathbf{Z}}, \mathfrak{e}^{1}, \mathfrak{e}^{2}\right)$ be as in Theorem 32 . If $\mu=3$ and the condition ( $\mathrm{g} 2)$ does not hold, then the weak envelope $\mathfrak{G}^{w}$ of $\mathfrak{G}$ is isomorphic to a pseudo-product $G L A$ of type $\left(X_{l}, \mathbf{s}, \lambda ; \mathfrak{b}, \nu ; L_{2}, \psi\right)$.

4.5. The prolongation of the negative part of the underlying GLAs of pseudoproduct GLAs.

In this subsection we investigate the prolongation of $\mathfrak{g}_{-}$for pseudo-product GLAs $\mathfrak{G}=\left(\mathfrak{g},\left(\mathfrak{g}_{p}\right)_{p \in \mathbf{Z}}, \mathfrak{e}^{1}, \mathfrak{e}^{2}\right)$ of type $\left(X_{l}, \mathbf{s}, \lambda ; \mathfrak{b}, \nu\right)$ and of type $\left(X_{l}, \mathbf{s}, \lambda ; \mathfrak{b}, \nu ; L_{2}, \psi\right)$.

4.5.1. Let $\mathfrak{G}=\left(\mathfrak{g},\left(\mathfrak{g}_{p}\right)_{p \in \mathbf{Z}}, \mathfrak{e}^{1}, \mathfrak{e}^{2}\right)$ be a pseudo-product GLA of type $\left(X_{l}, \mathbf{s}, \lambda\right.$; $\mathfrak{b}, \nu)$ of depth $\mu, \stackrel{\vee}{\mathfrak{G}}=\left(\stackrel{\vee}{\mathfrak{g}},\left(\stackrel{\vee}{\mathfrak{g}}_{p}\right)_{p \in \mathbf{Z}}, \mathfrak{e}^{1}, \mathfrak{e}^{2}\right)$ be the prolongation of $\mathfrak{G}_{-}$and $\mathcal{G}=$ $\bigoplus_{p \in \mathbf{Z}} \mathcal{G}_{p}$ be the prolongation of $\mathfrak{g}_{-}$. For convenience, we put $\mathfrak{d}_{p}=\mathfrak{d}_{p}(\mathcal{G})$. Also we use the same notation as in the construction of $\mathfrak{G}$. We let consider the cohomology group $H\left(\mathfrak{a}_{-1}, M\right)=\bigoplus_{p} H^{p}\left(\mathfrak{a}_{-1}, M\right)$ of the cochain complex $\left(C\left(\mathfrak{a}_{-1}, M\right)=\right.$ $\left.\bigoplus_{p} C^{p}\left(\mathfrak{a}_{-1}, M\right), \partial\right)$, where $C^{p}\left(\mathfrak{a}_{-1}, M\right)=\operatorname{Hom}\left(\Lambda^{p} \mathfrak{a}_{-1}, M\right)$ and the coboundary operator $\partial: C^{p}\left(\mathfrak{a}_{-1}, M\right) \rightarrow C^{p+1}\left(\mathfrak{a}_{-1}, M\right)$ is defined by

$$
(\partial \omega)\left(x_{1}, \ldots, x_{p+1}\right)=\sum_{i=1}^{p+1}(-1)^{i+1}\left[x_{i}, \omega\left(x_{1}, \ldots, \widehat{x}_{i}, \ldots, x_{p+1}\right)\right]
$$

for $\omega \in C^{p}\left(\mathfrak{a}_{-1}, M\right), x_{1}, \ldots, x_{p+1} \in \mathfrak{a}_{-1}$. Then the $\mathfrak{g}_{0}$-module structures on $\mathfrak{a}_{-1}$ and $M$ induce that on $C^{p}\left(\mathfrak{a}_{-1}, M\right)$ in the natural manner. Moreover since $\partial$ commutes with the action of the elements of $\mathfrak{g}_{0}$ on $C^{p}\left(\mathfrak{a}_{-1}, M\right)$, the $\mathfrak{g}_{0}$-module structure on $C^{p}\left(\mathfrak{a}_{-1}, M\right)$ induces that on $H^{p}\left(\mathfrak{a}_{-1}, M\right)$. For each integer $r$, let $C_{r}^{p}\left(\mathfrak{a}_{-1}, M\right)=$ $\operatorname{Hom}\left(\Lambda^{p} \mathfrak{a}_{-1}, M_{-p+r}\right)$. Then $\left(C_{r}\left(\mathfrak{a}_{-1}, M\right)=\bigoplus_{p} C_{r}^{p}\left(\mathfrak{a}_{-1}, M\right), \partial\right)$ is a subcomplex and the direct sum decomposition $C\left(\mathfrak{a}_{-1}, M\right)=\bigoplus_{r, p} C_{r}^{p}\left(\mathfrak{a}_{-1}, M\right)$ yields that of the cohomology group $H\left(\mathfrak{a}_{-1}, M\right)=\bigoplus H_{r}\left(\mathfrak{a}_{-1}, M\right)=\bigoplus H_{r}^{p}\left(\mathfrak{a}_{-1}, M\right)$. In particular, $H_{0}^{1}\left(\mathfrak{a}_{-1}, M\right)=\operatorname{Ker} \partial$. Furthermore $H_{r}^{p}\left(\mathfrak{a}_{-1}, M\right)$ is a $\mathfrak{g}_{0}$-submodule of $H^{p}\left(\mathfrak{a}_{-1}, M\right)$. Note that $H_{r}^{p}\left(\mathfrak{a}_{-1}, M\right)$ is the eigenspace with the eigenvalue $r$ with respect to the action of $E$ on $H^{p}\left(\mathfrak{a}_{-1}, M\right)$. For $\omega \in H_{0}^{1}\left(\mathfrak{a}_{-1}, M\right), x_{1}, x_{2} \in \mathfrak{a}_{-1}$, we have $0=$ 
$\partial \omega\left(x_{1}, x_{2}\right)=\left[x_{1}, \omega\left(x_{2}\right)\right]-\left[x_{2}, \omega\left(x_{1}\right)\right]$. We define a mapping $\iota$ of $H_{0}^{1}\left(\mathfrak{a}_{-1}, M\right)$ into $\mathfrak{d}_{0}$ as follows: $[\iota(\omega), x]=\omega(x),[\iota(\omega), y]=0$ for $\omega \in H_{0}^{1}\left(\mathfrak{a}_{-1}, M\right), x \in \mathfrak{a}_{-1}, y \in M$. Then $\iota$ is a monomorphism as a $\mathfrak{g}_{0}$-module. In what follows, we will identify $H_{0}^{1}\left(\mathfrak{a}_{-1}, M\right)$ with the subalgebra $\iota\left(H_{0}^{1}\left(\mathfrak{a}_{-1}, M\right)\right)$ of $\mathfrak{d}_{0}$; then $H_{0}^{1}\left(\mathfrak{a}_{-1}, M\right)=\left\{x \in \mathfrak{d}_{0}:\left[x, \mathfrak{e}^{1}\right] \subset \mathfrak{e}^{2}\right.$ and $\left.\left[x, \mathfrak{e}^{2}\right]=0\right\}$. In particular, if $\mu \geq 3$, then $H_{0}^{1}\left(\mathfrak{a}_{-1}, M\right)=\mathfrak{d}_{0}$.

Lemma 35. Suppose that $\mu=2, \operatorname{dim} \mathcal{G}=\infty$ and $H_{0}^{1}\left(\mathfrak{a}_{-1}, M\right)=\{0\}$. Then one of the following occurs:

(a) $\mathfrak{G}$ is of type $\left(C_{l}, \varepsilon_{l}, \varpi_{1}\right)(l \geq 2)$. In this case, $\mathcal{G}$ is isomorphic to $C H(l ; \mathbf{1} ; 3)$.

(b) $\mathfrak{G}$ is of type $\left(A_{l}, \varepsilon_{i}, \varpi_{1}\right)(l \geq 2, i<l)$. In this case, $\mathcal{G}$ is isomorphic to $W\left(l+1 ;\left(\mathbf{1}_{i}, 2 \mathbf{1}_{l-i+1}\right)\right)$.

Proof. Let $J$ be an element of $\mathfrak{z}(\mathfrak{l})$ such that $[J, x]=-x$ for $x \in M$. We set $\mathcal{L}_{k}=\{x \in \mathcal{G}:[J, x]=k x\}$ and $\mathcal{G}_{p, k}=\mathcal{G}_{p} \cap \mathcal{L}_{k}$; then $\mathcal{G}=\bigoplus_{p>-1} \mathcal{L}_{p}$ and $\mathcal{L}_{k}=\bigoplus_{p \in \mathbf{Z}} \mathcal{G}_{p, k}$. Clearly $\mathcal{G}_{-2,-1}=M_{-2}, \mathcal{G}_{-1,-1}=M_{-1}, \mathcal{G}_{-1,0}=\mathfrak{l}_{-1}$ and $\mathcal{G}_{-1}=$ $\mathcal{G}_{-1,-1} \oplus \mathcal{G}_{-1,0}$. Then $\mathcal{L}_{-1}=\mathcal{G}_{-1,-1} \bigoplus \mathcal{G}_{-2,-1}$. Indeed, $\left[\mathcal{G}_{0,-1}, \mathcal{G}_{-2,-1} \bigoplus \mathcal{G}_{-1,-1}\right] \subset$ $\mathcal{G}_{-2,-2} \bigoplus \mathcal{G}_{-1,-2}=\{0\}$ and $\left[\mathcal{G}_{0,-1}, \mathcal{G}_{-1,0}\right] \subset \mathcal{G}_{-1,-1}=M_{-1}$, so $\mathcal{G}_{0,-1} \subset H_{0}^{1}\left(\mathfrak{a}_{-1}, M\right)$ $=\{0\}$. Moreover by induction on $p$, we have $\mathcal{G}_{p,-1}=\{0\}$ for $p \geq 0$. Next we will prove that for $x \in \mathcal{L}_{k}(k \geq 0),\left[x, \mathcal{L}_{-1}\right]=\{0\}$ implies $x=0$. Let $W=$ $\left\{x \in \mathcal{L}_{k}:\left[x, \mathcal{L}_{-1}\right]=0\right\}$ and $\mathfrak{a}=\bigoplus_{p \in \mathbf{Z}} \mathfrak{a}_{p}$ be the graded ideal of $\mathcal{G}$ generated by $W$. Then $\mathfrak{a} \subset \bigoplus_{p \geq k} \mathcal{L}_{p}$ and $\mathfrak{a}=\bigoplus_{p, k \in \mathbf{Z}} \mathfrak{a}_{p, k}$, where $\mathfrak{a}_{p, k}=\mathfrak{a} \cap \mathcal{G}_{p, k}$. Further $\mathfrak{a}_{-1,0} \subset \mathfrak{l}_{-1}$ and $\left[\mathfrak{a}_{-1,0}, M\right] \subset \mathfrak{a}_{-3,-1} \bigoplus \mathfrak{a}_{-2,-1}=\{0\}$. Since the $\mathfrak{l}$-module $M$ is faithful, $\mathfrak{a}_{-1,0}=\{0\}$, so $\mathfrak{a} \subset \bigoplus_{p \geq 0} \mathcal{G}_{p}$. By transitivity of $\mathcal{G}$, we have $\mathfrak{a}=\{0\}$. From this fact, $\operatorname{dim} \mathcal{L}_{k}<\infty$ for all $k$ and $\mathcal{G}=\bigoplus_{k \geq-1} \mathcal{L}_{k}$ is a TGLA of depth one. Since $\mathfrak{l} \subset \mathcal{L}_{0}$ and since the $\mathfrak{l}$-module $M$ is irreducible, the TGLA $\mathcal{G}=\bigoplus_{k \geq-1} \mathcal{L}_{k}$ is irreducible. Hence since $\mathcal{G}$ is infinite dimensional, $\mathcal{G}=\bigoplus_{k>-1} \mathcal{L}_{k}$ is isomorphic to one of $W(n ; \mathbf{1}), C S(n ; \mathbf{1}), C H(n ; \mathbf{1} ; 2)(n \geq 2)$. In particular, $\mathcal{L}_{0}$ is reductive and $\left[\mathcal{L}_{0}, \mathcal{L}_{0}\right]$ is simple. Further since $\mathfrak{l}_{-1}=\mathcal{G}_{-1,0}$ and $\mathfrak{z}\left(\mathcal{L}_{0}\right) \subset \mathcal{G}_{0,0}$, we have $\mathfrak{l}=\mathcal{L}_{0}$. If $\mathcal{G}=\bigoplus_{k \geq-1} \mathcal{L}_{k}$ is isomorphic to $C S(n ; \mathbf{1}), \mathcal{G}=\bigoplus_{p \in \mathbf{Z}} \mathcal{G}_{p}$ is isomorphic to $C S\left(n ;\left(\mathbf{1}_{r}, 2 \mathbf{1}_{n-r}\right)\right)$. However since $W\left(n ;\left(\mathbf{1}_{r}, 2 \mathbf{1}_{n-r}\right)\right)_{-}=C S\left(n ;\left(\mathbf{1}_{r}, 2 \mathbf{1}_{n-r}\right)\right)_{-}$, it is impossible. Now suppose that $\mathcal{G}=\bigoplus_{k \geq-1} \mathcal{L}_{k}$ is isomorphic to $C H(n ; \mathbf{1} ; 2)$. Then $n=l, \mathfrak{l}$ is isomorphic to $\mathfrak{c s p}(l), \widehat{\mathfrak{l}}$ is a simple GLA of type $\left(C_{l}, \varepsilon_{l}\right)$ and the representation of $\mathfrak{l}$ on $M$ is elementary. Hence $\mathfrak{G}$ is of type $\left(C_{l}, \varepsilon_{l}, \varpi_{1}\right)$ and $\mathcal{G}=\bigoplus_{p \in \mathbf{Z}} \mathcal{G}_{p}$ is isomorphic to $C H(l ; \mathbf{1} ; 3)$. Next we suppose that $\mathcal{G}=\bigoplus_{k \geq-1} \mathcal{L}_{k}$ is isomorphic to $W(n ; \mathbf{1})$. Then $n=l+1, \mathfrak{l}$ is isomorphic to $\mathfrak{g l}(l+1), \mathfrak{l}$ is a simple GLA of type $\left(A_{l}, \varepsilon_{i}\right)$ and the representation of $\mathfrak{l}$ on $M$ is elementary. Hence $\mathfrak{G}$ is of type $\left(A_{l}, \varepsilon_{i}, \varpi_{1}\right)$ and $\mathcal{G}=\bigoplus_{p \in \mathbf{Z}} \mathcal{G}_{p}$ is isomorphic to $W\left(l+1 ;\left(\mathbf{1}_{i}, 2 \mathbf{1}_{l-i+1}\right)\right)$. However since the prolongation of $W\left(l+1 ;\left(\mathbf{1}_{l}, 2 \mathbf{1}_{1}\right)\right)_{-}$is isomorphic to $K(l ; \mathbf{1} ; 2)$, we have $i<l$ and $l \geq 2$. This proves our assertion.

From this lemma, the classification of $\mathfrak{G}$ such that $\operatorname{dim} \mathcal{G}=\infty$ is reduced to 
the case where $H_{0}^{1}\left(\mathfrak{a}_{-1}, M\right) \neq\{0\}$.

Let $\Delta$ be the root system of $(\mathfrak{l}, \mathfrak{h}), \Delta_{+}$be the set of all the positive roots of $\mathfrak{l}$ with respect to $\Pi_{1} \cup \Pi_{2}$, and $W$ be the Weyl group of $\mathfrak{l}$. We set $\Delta_{-}=\Delta \backslash \Delta_{+}$. For any $w \in W$, define $T_{w}=w \Delta_{+} \cap \Delta_{-}$. Let $n(w)$ be the number of elements in $T_{w}$, and $l(w)$ be the length of $w$, that is, the least non-negative integer $j$ such that $w$ can be written as $r_{i_{1}} \cdots r_{i_{j}}$, where $r_{i}$ is the element of $W$ defined by $r_{i}(x)=$ $x-\left\langle x, \alpha_{i}^{\vee}\right\rangle \alpha_{i}$ for $x \in \mathfrak{h}^{*}$. Then $l(w)=n(w)$ for all $w \in W$ (cf. [Bou68, Ch.6, no.6]). We set $W^{1}(1)=\left\{w \in W: n(w)=1, T_{w} \subset \Delta\left(\mathfrak{a}_{-1}\right)\right\}$, where $\Delta\left(\mathfrak{a}_{-1}\right)=$ $\left\{\alpha \in \Delta: e_{\alpha} \in \mathfrak{a}_{-1}\right\}$. Then there is a $j$ such that $W^{1}(1)=\left\{r_{j}\right\}$. On the other hand, we know that $T_{r_{j}}=\left\{-\alpha_{j}\right\}$, so $-\alpha_{j} \in \Delta\left(\mathfrak{a}_{-1}\right)$. Thus $W^{1}(1)=\left\{r_{i_{0}}\right\}$. By Kostant's theorem [Kos61, Theorem 5.14], $H^{1}\left(\mathfrak{a}_{-1}, M\right)$ is an irreducible $\mathfrak{g}_{0}$-module with lowest weight $\xi:=r_{i_{0}}\left(w_{0}(\lambda+\nu)\right)+\alpha_{i_{0}}+\sigma$. Then $H_{0}^{1}\left(\mathfrak{a}_{-1}, M\right) \neq\{0\}$ if and only if $\langle\xi, E\rangle=0$. Since $\langle\lambda+\nu+\sigma, E\rangle=-1, E_{1}=\varpi_{i_{0}}^{\vee}$ and $E_{2} \in \mathfrak{z}$, we have $\langle\xi, E\rangle=\left\langle w_{0}(\lambda)-\lambda, \varpi_{i_{0}}^{\vee}\right\rangle-\left\langle w_{0}(\lambda), \alpha_{i_{0}}^{\vee}\right\rangle$.

Now we seek the weight $\lambda$ such that $\langle\xi, E\rangle=0$ case by case. This will be done by using the table of [Bou68]. Also, we put $\lambda=\sum_{i=1}^{l} m_{i} \varpi_{i}\left(m_{i} \in \mathbf{Z}_{+}\right)$.

Case 1. Take $\left(X_{l}, \mathbf{s}\right)=\left(A_{l}, \varepsilon_{i}\right)(i \leq[(l+1) / 2])$. Then

$$
\langle\xi, E\rangle=-\sum_{j=1}^{i} j m_{j}-\sum_{j=i+1}^{l-i} i m_{j}-\sum_{j=l-i+2}^{l}(l-j+1) m_{j}-(i-1) m_{l-i+1} .
$$

Hence if $\langle\xi, E\rangle=0$, then $i=1$ and $\lambda=m_{l} \varpi_{l}\left(m_{l} \geq 1\right)$.

Case 2. Take $\left(X_{l}, \mathbf{s}\right)=\left(B_{l}, \varepsilon_{1}\right)(l \geq 3)$. Then

$$
\langle\xi, E\rangle=-m_{1}-2 \sum_{j=2}^{l-1} m_{j}-m_{l} \neq 0 .
$$

Case 3. Take $\left(X_{l}, \mathbf{s}\right)=\left(C_{l}, \varepsilon_{l}\right)(l \geq 2)$. Then

$$
\langle\xi, E\rangle=\sum_{j=1}^{l-1} j m_{j}-(l-1) m_{l} \neq 0 .
$$

Case 4. Take $\left(X_{l}, \mathbf{s}\right)=\left(D_{l}, \varepsilon_{1}\right)(l \geq 4)$. Then

$$
\langle\xi, E\rangle=-m_{1}-2 \sum_{j=2}^{l-2} m_{j}-m_{l-1}-m_{l} \neq 0 .
$$

Case 5. take $\left(X_{l}, \mathbf{s}\right)=\left(D_{l}, \varepsilon_{l-1}\right)(l \geq 5, l$ is an even number $)$. Then

$$
\langle\xi, E\rangle=-\sum_{j=1}^{l-2} j m_{j}-(1 / 2)(l-2) m_{l-1}-(1 / 2)(l-2) m_{l} \neq 0 .
$$


Case 6. Take $\left(X_{l}, \mathbf{s}\right)=\left(D_{l}, \varepsilon_{l-1}\right)(l \geq 5, l$ is an odd number $)$. Then

$$
\langle\xi, E\rangle=-\sum_{j=1}^{l-2} j m_{j}-(1 / 2)(l-1) m_{l-1}-(1 / 2)(l-3) m_{l} \neq 0 .
$$

Case 7. Take $\left(X_{l}, \mathbf{s}\right)=\left(E_{6}, \varepsilon_{1}\right)$. Then

$$
\langle\xi, E\rangle=-2 m_{1}-2 m_{2}-3 m_{3}-4 m_{4}-3 m_{5}-m_{6} \neq 0 .
$$

Case 8. Take $\left(X_{l}, \mathbf{s}\right)=\left(E_{7}, \varepsilon_{7}\right)$. Then

$$
\langle\xi, E\rangle=-2 m_{1}-3 m_{2}-4 m_{3}-6 m_{4}-5 m_{5}-4 m_{6}-2 m_{7} \neq 0 .
$$

Thus, if $H_{0}^{1}\left(\mathfrak{a}_{-1}, M\right) \neq\{0\}$, then the triple $\left(X_{l}, \mathbf{s}, \lambda\right)$ is $\left(A_{l}, \varepsilon_{1}, k \varpi_{l}\right)(k \geq 1)$. On the other hand, if $\mathfrak{G}$ is of type $\left(A_{l}, \varepsilon_{1}, k \varpi_{l}\right)\left(\operatorname{resp} .\left(A_{l}, \varepsilon_{1}, k \varpi_{l} ; A_{n}, \varpi_{1}\right)\right)$, then $\mathfrak{G}$ is a pseudo-projective GLA of order $k+1$ of bidegree $(l+1, l)($ resp. $(l+n+1, l))$, so $\mathcal{G}$ is isomorphic to $K(l ; \mathbf{1} ; k+1)$ (resp. $W\left(l+n+1 ;\left(\mathbf{1}_{l},(k+1) \mathbf{1}_{n+1}\right)\right)$ ) (cf. 1.5). In particular, $\mathcal{G}$ is infinite dimensional. Also, we remark that if $\mathfrak{G}$ is of type $\left(A_{l}, \varepsilon_{i}, \varpi_{1}\right)$, then the system $\mathfrak{G}^{\prime}=\left(\mathfrak{g},\left(\mathfrak{g}_{p}\right)_{p \in \mathbf{Z}}, \mathfrak{e}^{2}, \mathfrak{e}^{1}\right)$ is a pseudo-projective GLA of order 2 of bidegree $(l+1, i)$. From Theorem 33 and Lemma 35, we obtain the following theorem.

Theorem 36. Let $\mathfrak{G}=\left(\mathfrak{g},\left(\mathfrak{g}_{p}\right)_{p \in \mathbf{Z}}, \mathfrak{e}^{1}, \mathfrak{e}^{2}\right)$ be a pseudo-product GLA of type $\left(X_{l}, \mathbf{s}, \lambda ; \mathfrak{b}, \nu\right)$ and $\stackrel{\vee}{\mathfrak{G}}=\left(\stackrel{\vee}{\mathfrak{g}},\left(\mathfrak{g}_{p}\right)_{p \in \mathbf{Z}}, \mathfrak{e}^{1}, \mathfrak{e}^{2}\right)$ be the prolongation of $\mathfrak{G}_{-}$. Assume that $\mathfrak{g}_{0}=\mathfrak{g}_{0}$ and the prolongation $\mathcal{G}$ of $\mathfrak{g}_{-}$is infinite dimensional. Then one of the following occurs:

(a) $\mathfrak{G}$ is of type $\left(A_{l}, \varepsilon_{1}, \varpi_{l}\right)$. In this case, $\mathfrak{G}$ is isomorphic to $K(l ; \mathbf{1} ; 2), \stackrel{\vee}{\mathfrak{G} \neq}$ $\mathfrak{G}$, and $\mathfrak{G}$ is a simple GLA of type $\left(A_{l+1}, \varepsilon_{1}+\varepsilon_{l+1}\right)$.

(b) $\mathfrak{G}$ is of type $\left(A_{l}, \varepsilon_{1}, 2 \varpi_{l}\right)$. In this case, $\mathcal{G}$ is isomorphic to $K(l ; \mathbf{1} ; 3), \stackrel{\vee}{\mathfrak{G}}$ $\neq \mathfrak{G}$, and $\mathfrak{g}$ is a simple $G L A$ of type $\left(C_{l+1}, \varepsilon_{1}+\varepsilon_{l+1}\right)$.

(c) $\mathfrak{G}$ is of type $\left(A_{l}, \varepsilon_{1}, k \varpi_{l}\right)(k \geq 3)$. In this case, $\mathfrak{G}$ is isomorphic to $K(l ; \mathbf{1} ; k+1)$ and $\mathfrak{G}=\mathfrak{G}$.

(d) $\mathfrak{G}$ is of type $\left(A_{l}, \varepsilon_{1}, \varpi_{l} ; A_{n}, \varpi_{1}\right)$. In this case, $\mathfrak{G}$ is isomorphic to $W(l+$ $\left.n+1 ;\left(\mathbf{1}_{l}, 2 \mathbf{1}_{n+1}\right)\right), \mathfrak{G} \neq \mathfrak{G}$, and $\stackrel{\vee}{\mathfrak{g}}$ is a simple GLA of type $\left(A_{l+n+1}, \varepsilon_{1}+\varepsilon_{l+1}\right)$.

(e) $\mathfrak{G}$ is of type $\left(A_{l}, \varepsilon_{1}, k \varpi_{l} ; A_{n}, \varpi_{1}\right)(k \geq 2)$. In this case, $\mathcal{G}$ is isomorphic to $W\left(l+n+1 ;\left(\mathbf{1}_{l},(k+1) \mathbf{1}_{n+1}\right)\right)$ and $\mathfrak{G}=\mathfrak{G}$.

(f) $\mathfrak{G}$ is of type $\left(A_{l}, \varepsilon_{i}, \varpi_{1}\right)(l \geq 2, i>l)$. In this case, $\mathcal{G}$ is isomorphic to $W\left(l+1 ;\left(\mathbf{1}_{i}, 2 \mathbf{1}_{l-i+1}\right)\right), \stackrel{\vee}{\mathfrak{G}} \neq \mathfrak{G}$ and $\stackrel{\vee}{\mathfrak{g}}$ is a simple GLA of type $\left(A_{l+1}, \varepsilon_{1}+\varepsilon_{i+1}\right)$. 
(g) $\mathfrak{G}$ is of type $\left(C_{l}, \varepsilon_{l}, \varpi_{1}\right)$. In this case, $\mathcal{G}$ is isomorphic to $C H(l ; \mathbf{1} ; 3)$ and $\stackrel{\vee}{\mathfrak{G}}=\mathfrak{G}$.

Proof. By the above results and Lemma 35, we have only to investigate the type of $\stackrel{\vee}{\mathfrak{g}}$. Now $\mathfrak{G}$ be one of types $\left(A_{l}, \varepsilon_{1}, k \varpi_{l}\right)(k \geq 3),\left(A_{l}, \varepsilon_{1}, k \varpi_{l} ; A_{n}, \varpi_{1}\right)(k \geq 2)$, $\left(C_{l}, \varepsilon_{l}, \varpi_{1}\right)$. By [Yat92, Theorem 16], $\mathcal{G}=\bigoplus_{p \in \mathbf{Z}} \mathcal{G}_{p}$ cannot be the prolongation of the negative part of a finite dimensional simple GLA. Thus $\stackrel{\vee}{\mathfrak{g}}$ is not simple, so $\stackrel{\vee}{\mathfrak{G}} \neq \mathfrak{G}$. Next let $\mathfrak{G}$ be of type $\left(A_{l}, \varepsilon_{1}, 2 \varpi_{l}\right)$ (resp. $\left.\left(A_{l}, \varepsilon_{1}, \varpi_{l} ; A_{n}, \varpi_{1}\right)\right)$ and $\mathfrak{s}=\bigoplus_{p \in \mathbf{Z}^{\mathfrak{s}} p}$ be a simple GLA of type $\left(C_{l+1}, \varepsilon_{1}+\varepsilon_{l+1}\right)$ (resp. $\left.\left(A_{l+n+1}, \varepsilon_{1}+\varepsilon_{l+1}\right)\right)$. Using the notation as in 4.4.1 for $\mathfrak{s}$, we define subsets $\Xi_{i}(i=1,2)$ of a root system $\Delta$ of $\mathfrak{s}$ by $\Xi_{1}=\left\{\alpha=\sum k_{i} \alpha_{i} \in \Delta_{-1}: k_{i_{1}}=-1\right.$ and $\left.k_{i_{2}}=0\right\}, \Xi_{2}=\Delta_{-1} \backslash \Xi_{1}$, where $i_{1}=1$ and $i_{2}=l+1$. Further we put $\mathfrak{f}^{i}=\sum_{\alpha \in \Xi_{i}} \mathbf{C} e_{\alpha}(i=1,2)$. By an immediate inspection, we obtain that the system $\mathfrak{S}=\left(\mathfrak{s},\left(\mathfrak{s}_{p}\right)_{p \in \mathbf{Z}}, \mathfrak{f}^{1}, \mathfrak{f}^{2}\right)$ is a pseudo-projective GLA of order 3 (resp. 2) of bidegree $(l+1, l)$ (resp. $(l+n+1, l))$. Since $\mathfrak{S}$ is the prolongation of $\mathfrak{S}_{-}$(cf. Remark in 4.1), we conclude that the prolongation of $\mathfrak{G}_{-}$ coincides with $\mathfrak{S}$. Finally let $\mathfrak{G}$ be of type $\left(A_{l}, \varepsilon_{i}, \varpi_{1}\right)(1 \leq i \leq l)$ and $\mathfrak{s}=\bigoplus_{p \in \mathbf{Z}} \mathfrak{s}_{p}$ be a simple GLA of type $\left(A_{l+1}, \varepsilon_{1}+\varepsilon_{i+1}\right)$. Note that a pseudo-product GLA of type $\left(A_{l}, \varepsilon_{1}, \varpi_{l}\right)$ is isomorphic to that of type $\left(A_{l}, \varepsilon_{l}, \varpi_{1}\right)$. In the above arguments, considering the case $i_{1}=1$ and $i_{2}=i+1$, we can construct a simple pseudoprojective GLA $\mathfrak{S}$ of order 2 of bidegree $(l+1, i)$. Thus the prolongation of $\mathfrak{G}_{-}$is isomorphic to $\mathfrak{S}$. This proves our assertion.

Finally we investigate whether $\mathcal{G}$ coincides with $\mathfrak{g}$ in case $\operatorname{dim} \mathcal{G}<\infty$.

Proposition 37. Let $\mathfrak{G}, \stackrel{\vee}{\mathfrak{G}}$ and $\mathcal{G}=\bigoplus_{p \in \mathbf{Z}} \mathcal{G}_{p}$ be as above. If $\operatorname{dim} \mathcal{G}<\infty$, then $\mathcal{G}$ coincides with $\stackrel{\vee}{\mathfrak{g}}$.

Proof. We use the same notation as in the proof of Lemma 35. For $|k| \geq$ $2,\left[\mathcal{G}_{0, k}, \mathfrak{e}^{i}\right]=\{0\}(i=1,2)$, so $\mathcal{G}_{0, k}=\{0\}$ by transitivity. Moreover $\left[\mathcal{G}_{0,-1}, \mathfrak{e}^{1}\right] \subset \mathfrak{e}^{2}$ and $\left[\mathcal{G}_{0,-1}, \mathfrak{e}^{2}\right]=\left[\mathcal{G}_{0,-1}, \mathfrak{g}_{p}\right]=\{0\}$ for $p \leq-2$, so $\mathcal{G}_{0,-1} \subset H_{0}^{1}\left(\mathfrak{a}_{-1}, M\right)$. From the proof of Theorem 36 , we have $\mathcal{G}_{0,-1}=\{0\}$. Suppose that $\mathcal{G}$ is semisimple. Since $\mathcal{G}_{0}$ is reductive and $\mathcal{G}_{0,1}$ is a nilpotent ideal of $\mathcal{G}_{0}$, we have $\mathcal{G}_{0,1}=\{0\}$, so $\mathcal{G}_{0}=\mathcal{G}_{0,0}$. In this case, since $\mathcal{G}_{0}=\mathfrak{v g}_{0}$, we have $\mathcal{G}=\mathfrak{v}$. Next suppose that $\mathcal{G}$ is not semisimple. Hence there is a commutative graded ideal $\mathfrak{u}=\bigoplus_{p \in \mathbf{Z}} \mathfrak{u}_{p}$ of $\mathcal{G}$; then $\mathfrak{u}_{-1}=\mathfrak{e}^{2}$ and $\mathfrak{u}_{p}=\mathfrak{g}_{p}$ for $p \leq-2$. Therefore $\left[\mathcal{G}_{0,1}, \mathfrak{e}^{2}\right] \subset \mathfrak{e}^{1} \cap \mathfrak{e}^{2}=\{0\}$ and $\left[\mathcal{G}_{0,1}, \mathfrak{e}^{1}\right]=\{0\}$. By transitivity, we have $\mathcal{G}_{0,1}=\{0\}$, so $\mathcal{G}_{0}=\mathfrak{g}_{0}$. Thus we know that $\mathcal{G}=\stackrel{\vee}{\mathfrak{g}}$.

4.5.2. Let $\mathfrak{G}=\left(\mathfrak{g},\left(\mathfrak{g}_{p}\right)_{p \in \mathbf{Z}}, \mathfrak{e}^{1}, \mathfrak{e}^{2}\right)$ be a pseudo-product GLA of type $\left(X_{l}, \mathbf{s}, \lambda\right.$; $\left.\mathfrak{b}, \nu ; L_{2}, \psi\right)$, and $\stackrel{\vee}{\mathfrak{G}}$ and $\mathcal{G}$ be as in 4.5.1. For $p \in \mathbf{Z}$, we set $\mathfrak{f}_{p}=\left\{x \in \mathcal{G}_{p}:\left[x, \mathfrak{g}_{-3}\right] \subset\right.$ $\left.\mathfrak{g}_{-3}\right\}$. Then $\mathfrak{f}:=\bigoplus_{p \in \mathbf{Z}} \mathfrak{f}_{p}$ is a TGLA such that $\mathfrak{g}_{-3}$ is an ideal of $\mathfrak{f}$. In order that $\mathcal{G}$ be infinite dimensional, it is necessary and sufficient that $\mathfrak{f}$ is infinite dimensional. 


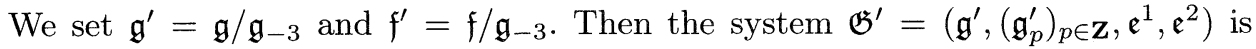
a pseudo-product GLA of depth 2 of type $\left(X_{l}, \mathbf{s}, \lambda ; \mathfrak{b}, \nu\right)$ and $\mathfrak{f}^{\prime}$ is contained in the prolongation $\mathcal{G}^{\prime}=\bigoplus_{p \in \mathbf{Z}} \mathcal{G}_{p}^{\prime}$ of $\mathfrak{g}_{-}^{\prime}$. By Theorem 35 and $(4.2)$, if $\operatorname{dim} \mathcal{G}=\infty$, then $\mathfrak{G}^{\prime}$ is of type $\left(A_{1}, \varepsilon_{1}, \varpi_{1} ; \mathfrak{b}, \nu\right)$ or $\left(C_{l}, \varepsilon_{l}, \varpi_{1}\right)$. In case $\mathfrak{G}^{\prime}$ is of type $\left(A_{1}, \varepsilon_{1}, \varpi_{1} ; \mathfrak{b}, \nu\right), \mathfrak{G}$ is a pseudo-projective GLA of order 2 of bidegree $(n+1,1)$, where $n=\operatorname{dim} E(\nu+\sigma)$. Since $\mathfrak{d}_{0}(\mathcal{G}) \subset \mathfrak{d}_{0}\left(\mathcal{G}^{\prime}\right)$ and $\left[\mathfrak{d}_{0}(\mathcal{G}), \mathfrak{e}^{2}\right] \subset \mathfrak{e}^{1}$, only the case $n=1$ is possible, so $(\mathfrak{b}, \nu)=(\{0\}, 0)$. On the other hand, if $\operatorname{dim} \mathcal{G}<\infty$, then $\stackrel{v}{\mathfrak{g}}=\mathcal{G}$. This result can be proved by the same method as in the proof of Prop. 36. Thus we obtain the following theorem.

Theorem 38. Let $\mathfrak{G}$ be a pseudo-product GLA of type $\left(X_{l}, \mathbf{s}, \lambda ; \mathfrak{b}, \nu ; L_{2}, \psi\right)$, $\stackrel{\vee}{\mathfrak{G}}=\left(\stackrel{\vee}{\mathfrak{g}},\left(\stackrel{\vee}{\mathfrak{g}_{p}}\right)_{p \in \mathbf{Z}}, \mathfrak{e}^{1}, \mathfrak{e}^{2}\right)$ be the prolongation of $\mathfrak{G}_{-}$and $\mathcal{G}=\bigoplus_{p \in \mathbf{Z}} \mathcal{G}_{p}$ be the prolongation of $\mathfrak{g}_{-}$. Then:

(1) If $\operatorname{dim} \mathcal{G}=\infty$, then $\mathfrak{G}$ is of type $\left(C_{l}, \varepsilon_{l}, \varpi_{1} ; 0\right)(l \geq 1)$. In this case,

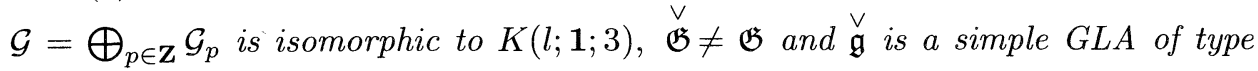
$\left(C_{l+1}, \varepsilon_{1}+\varepsilon_{l+1}\right)$.

(2) If $\operatorname{dim} \mathcal{G}<\infty$, then $\stackrel{\vee}{\mathfrak{g}}=\mathcal{G}$.

4.6. Derivations of the underlying Lie algebras of pseudo-product GLAs.

In this subsection, we will give a necessary and sufficient condition that the derivations of pseudo-product GLA of type $(\mathfrak{l}, M)$ or of the irreducible type $(\mathfrak{l}, M, N)$ are all inner.

4.6.1. Let $\mathfrak{G}=\left(\mathfrak{g},\left(\mathfrak{g}_{p}\right)_{p \in \mathbf{Z}}, \mathfrak{e}^{1}, \mathfrak{e}^{2}\right)$ be a pseudo-product GLA of type $(\mathfrak{l}, M)$. Assume that $\mathfrak{G}^{w}=\mathfrak{G}$. We decompose $M$ into a direct sum of the isotypical components as follows: $M=\bigoplus_{\lambda \in \mathfrak{S}} M^{\lambda}$. By Lemma 29, there is an element $J_{\lambda}$ of $\mathfrak{z}(\mathfrak{l})$ such that $\left[J_{\lambda}, m_{\mu}\right]=-\delta_{\lambda \mu} m_{\mu}$ for $m_{\mu} \in M^{\mu}$. We set $\mathfrak{z}=\sum_{\lambda \in \mathfrak{S}} F J_{\lambda}$. For $\eta \in \mathfrak{z}^{*}$, we write $\mathfrak{g}^{\eta}=\{x \in \mathfrak{g}:[h, x]=\langle\eta, h\rangle x$ for all $h \in \mathfrak{z}\}$ and $\operatorname{Der}(\mathfrak{g})_{\eta}=\left\{D \in \operatorname{Der}(\mathfrak{g}): D\left(\mathfrak{g}^{\xi}\right) \subset\right.$ $\mathfrak{g}^{\xi+\eta}$ for all $\left.\xi \in \mathfrak{z}^{*}\right\}$. Then $\mathfrak{g}=\bigoplus_{\eta \in \mathfrak{z}^{*}} \mathfrak{g}^{\eta}$ and $\operatorname{Der}(\mathfrak{g})=\bigoplus_{\eta \in \mathfrak{z}^{*}} \operatorname{Der}(\mathfrak{g})_{\eta}$ (cf. [Far88, Prop.1.1]). Let $D \in \operatorname{Der}(\mathfrak{g})_{\eta}(\eta \neq 0)$. Choose $h \in \mathfrak{z}$ such that $\langle\eta, h\rangle=1$; then $D=\operatorname{ad} D h$. Thus Der $(\mathfrak{g})=\operatorname{Der}(\mathfrak{g})_{0}+\operatorname{ad} \mathfrak{g}$. Since Der $(\mathfrak{g})_{0} \cdot \mathfrak{l} \subset \mathfrak{l}$, ad $\mathfrak{l}$ is an ideal of $\operatorname{Der}(\mathfrak{g})_{0}$. Also we can easily prove that the $\operatorname{Der}(\mathfrak{g})_{0^{-}}$-module $M^{\lambda}$ is irreducible, so $\operatorname{Der}(\mathfrak{g})_{0}$ is reductive. Therefore there is a semisimple ideal $\mathfrak{u}$ of Der $(\mathfrak{g})_{0}$ such that $\operatorname{Der}(\mathfrak{g})_{0}=\operatorname{ad} \mathfrak{l} \bigoplus \mathfrak{u}$. For $\varphi \in \mathcal{L}_{\mathfrak{l}}(M)$, we define $D_{\varphi} \in \operatorname{Der}(\mathfrak{g})_{0}$ as follows: $D_{\varphi}(\mathfrak{l})=0$, $D_{\varphi}(m)=\varphi(m)$ for $m \in M$. Since $\operatorname{ad} \mathfrak{z}(\mathfrak{l}) \bigoplus \mathfrak{u}=\left\{D \in \operatorname{Der}(\mathfrak{g})_{0}: D(\mathfrak{l})=0\right\}$, we obtain that $D_{\varphi} \in \operatorname{ad} \mathfrak{z}(\mathfrak{l}) \oplus \mathfrak{u}$ for all $\varphi \in \mathcal{L}_{\mathfrak{l}}(M)$. Then a linear mapping $\Psi$ of $\mathcal{L}_{\mathfrak{l}}(M)$ into ad $\mathfrak{z}(\mathfrak{l}) \bigoplus \mathfrak{u}$ defined by $\Psi(\varphi)=D_{\varphi}$ is a Lie algebra isomorphism. By the definition of $\mathfrak{G}, \Psi\left(\mathfrak{z}\left(\mathcal{L}_{\mathfrak{l}}(M)\right)\right)=\operatorname{ad} \mathfrak{z}(\mathfrak{l})$. Let $M_{1}^{\lambda}$ be an irreducible l-submodule of $M^{\lambda}$, and we set $C^{\lambda}=\mathcal{L}_{\mathfrak{l}}\left(M_{1}^{\lambda}\right)$. Then $\mathcal{L}_{\mathfrak{l}}(M)$ is isomorphic to $\prod_{\lambda \in \mathfrak{S}} \mathfrak{g l}\left(n_{\lambda} ; C^{\lambda}\right)$, where $n_{\lambda}$ is the length of the $\mathfrak{l}$-module of $\dot{M}^{\lambda}$. Hence $\mathfrak{u}$ is isomorphic to $\prod_{\lambda \in \mathfrak{S}} \mathfrak{s l}\left(n_{\lambda} ; C^{\lambda}\right)$. Consequently, in case $\mathfrak{G}^{w}=\mathfrak{G}$, in order that $\operatorname{ad} \mathfrak{g}=\operatorname{Der}(\mathfrak{g})$, it is necessary and sufficient that the following condition holds: 
(A1) $\operatorname{dim} \mathfrak{z}(\mathfrak{l})$ is equal to the length of the $\mathfrak{l}$-module $M$ (resp. the $\mathfrak{l}^{\mathbf{C}}$-module $M^{\mathbf{C}}$ ) in case $F=\mathbf{C}$ (resp. $F=\mathbf{R}$ ).

Note that if either the condition "ad $\mathfrak{g}=\operatorname{Der}(\mathfrak{g})$ " or the condition (A1) holds, then $\mathfrak{G}^{w}=\mathfrak{G}$. Thus we obtain the following proposition.

Proposition 39. Let $\mathfrak{G}=\left(\mathfrak{g},\left(\mathfrak{g}_{p}\right)_{p \in \mathbf{Z}}, \mathfrak{e}^{1}, \mathfrak{e}^{2}\right)$ be a pseudo-product GLA of type $(\mathfrak{l}, M)$. Then $\operatorname{Der}(\mathfrak{g})=\operatorname{ad} \mathfrak{g}$ if and only if the condition (A1) holds.

4.6.2. Let $\mathfrak{G}=\left(\mathfrak{g},\left(\mathfrak{g}_{p}\right)_{p \in \mathbf{Z}}, \mathfrak{e}^{1}, \mathfrak{e}^{2}\right)$ be a pseudo-product GLA of the irreducible type $(\mathfrak{l}, M, N)$. Assume that $\mathfrak{G}^{w}=\mathfrak{G}$. By Lemma 31 , there is an element $J$ of $\mathfrak{z}(\mathfrak{l})$ such that $[J, m]=-m$ for $m \in M$. We set $\mathfrak{z}=F J$. For $\eta \in \mathfrak{z}^{*}$, we define $\mathfrak{g}^{\eta}$ and $\operatorname{Der}(\mathfrak{g})_{\eta}$ as in 4.6.1; then Der $(\mathfrak{g})=\operatorname{Der}(\mathfrak{g})_{0}+\operatorname{ad} \mathfrak{g}$. Since Der $(\mathfrak{g})_{0} \cdot \mathfrak{l} \subset \mathfrak{l}$, ad $\mathfrak{l}$ is an ideal of $\operatorname{Der}(\mathfrak{g})_{0}$. Since the $\operatorname{Der}(\mathfrak{g})_{0}$-module $M$ is faithful and irreducible, $\operatorname{Der}(\mathfrak{g})_{0}$ is reductive. Therefore there is a semisimple ideal $\mathfrak{u}$ of $\operatorname{Der}(\mathfrak{g})_{0}$ such that $\operatorname{Der}(\mathfrak{g})_{0}=\operatorname{adl} \bigoplus \mathfrak{u}$. We set $\mathcal{L}_{\mathfrak{l}}(M: N)=\left\{\varphi \in \mathcal{L}_{\mathfrak{l}}(M): d_{\varphi}^{2}(N) \subset N\right\}$ and $L=\Lambda^{2}(M) / N$. Let $\Phi$ be the projection of $\Lambda^{2}(M)$ onto $L$. For $\varphi \in \mathcal{L}_{\mathfrak{l}}(M: N)$, there is a $d_{\varphi}^{2} \in \mathcal{L}_{\mathfrak{l}}(L)$ such that $d_{\varphi}^{2} \circ \Phi=\Phi \circ d_{\varphi}^{2}$, and we define $D_{\varphi} \in \operatorname{Der}(\mathfrak{g})_{0}$ as follows: $D_{\varphi}(\mathfrak{l})=0, D_{\varphi}\left|M=\varphi, D_{\varphi}\right| L=d_{\varphi}^{2}$. Then a linear mapping $\Psi$ of $\mathcal{L}_{\mathfrak{l}}(M: N)$ into $\operatorname{ad} \mathfrak{z}(\mathfrak{l}) \bigoplus \mathfrak{u}$ defined by $\Psi(\varphi)=D_{\varphi}$ is a Lie algebra isomorphism. Moreover $\Psi(\mathfrak{z} N)=\operatorname{ad} \mathfrak{z}(\mathfrak{l})$. Consequently, in case $\mathfrak{G}^{w}=\mathfrak{G}$, in order that $\operatorname{ad} \mathfrak{g}=\operatorname{Der}(\mathfrak{g})$, it is necessary and sufficient that the following condition holds:

(A2) $\quad \operatorname{dim} \mathfrak{z}(\mathfrak{l})=\operatorname{dim} \mathcal{L}_{\mathfrak{l}}(M: N)$.

Note that if either the condition "ad $\mathfrak{g}=\operatorname{Der}(\mathfrak{g})$ " or the condition (A2) holds, then $\mathfrak{G}^{w}=\mathfrak{G}$. Thus we obtain the following proposition.

Proposition 40. Let $\mathfrak{G}=\left(\mathfrak{g},\left(\mathfrak{g}_{p}\right)_{p \in \mathbf{Z}}, \mathfrak{e}^{1}, \mathfrak{e}^{2}\right)$ be a pseudo-product GLA of the irreducible type $(\mathfrak{l}, M, N)$. Then $\operatorname{Der}(\mathfrak{g})=\operatorname{ad} \mathfrak{g}$ if and only if the condition (A2) holds.

4.7. Automorphisms of the underlying Lie algebras of pseudo-product GLAs.

In this subsection, we assume that the ground field $F$ is the field $\mathbf{C}$ of complex numbers. Let $\mathfrak{G}=\left(\mathfrak{g},\left(\mathfrak{g}_{p}\right)_{p \in \mathbf{Z}}, \mathfrak{e}^{1}, \mathfrak{e}^{2}\right)$ be a pseudo-product GLA of type $(\mathfrak{l}, M)$ such that $\operatorname{Der}(\mathfrak{g})=\operatorname{ad} \mathfrak{g}$. Let Aut $(\mathfrak{g})$ be the automorphism group of $\mathfrak{g}$ and Int $(\mathfrak{g})$ be the adjoint group of $\mathfrak{g}$. Since $M$ is the largest nilpotent ideal of $\mathfrak{g}$, every element of Aut $(\mathfrak{g})$ preserves $M$. Let Aut $(\mathfrak{g}, \mathfrak{l})$ be the group of automorphisms of $\mathfrak{g}$ preserving $\mathfrak{l}$. As in [Tan85, Lemma 1.6], Aut $(\mathfrak{g})$ is a semidirect product of Aut $(\mathfrak{g}, \mathfrak{l})$ by $\exp (\operatorname{ad} M)$. We shall investigate the structure of $\operatorname{Aut}(\mathfrak{g}, \mathfrak{l})$.

Let $\mathfrak{h}, \Delta$ and $\Pi$ be a Cartan subalgebra of $\mathfrak{l}$, a root system of $(\mathfrak{l}, \mathfrak{h})$ and a simple root system of $(\mathfrak{l}, \mathfrak{h}, \Delta)$ respectively. By Prop. 39, we can decompose the $\mathfrak{l}$-module $M$ into a direct sum of irreducible l-modules $M=\bigoplus_{i=1}^{m} M^{(i)}$ such that each $M^{(i)}$ is not isomorphic to each other as an $\mathfrak{l}$-module. Let $\Lambda_{i}$ be the highest weight of the irreducible l-module $M^{(i)}$ and $F_{\Lambda_{i}}$ be the corresponding weight vector. Also we set $\mathfrak{l}^{\prime}=[\mathfrak{l}, \mathfrak{l}]$. Let $\varphi \in \operatorname{Aut}(\mathfrak{g}, \mathfrak{l})$. By [Bou75, Ch.VIII, §5, no.3, Cor.1 to Prop.5], 
there is a $\psi \in \operatorname{Int}\left(\mathfrak{l}^{\prime}\right)$ such that $\psi \circ\left(\varphi \mid \mathfrak{l}^{\prime}\right)$ is a diagram automorphism of $\mathfrak{l}^{\prime}$ with respect to $\left(\mathfrak{l}^{\prime}, \mathfrak{l}^{\prime} \cap \mathfrak{h}, \Pi\right)$. The automorphism $\psi$ can be naturally extended to an inner automorphism of $\mathfrak{g}$, which is also denoted by $\psi$. Since $\psi \circ \varphi\left(F_{\Lambda_{i}}\right)$ is the highest weight vector of $M$, there is a permutation $\sigma$ of order $m$ such that ${ }^{t}(\psi \circ \varphi \mid \mathfrak{h})^{-1} \Lambda_{i}=$ $\Lambda_{\sigma(i)}$. Also there is an element $\tau$ of $\exp (\operatorname{ad}(\mathfrak{z}))$ such that $\tau \circ \psi \circ \varphi\left(F_{\Lambda_{i}}\right)=F_{\Lambda_{\sigma(i)}}$. We set $\Sigma=\left\{\Lambda_{1}, \ldots, \Lambda_{m}\right\}$ and $Q_{\Sigma}=\left\{F_{\Lambda_{1}}, \ldots, F_{\Lambda_{m}}\right\}$. Let $G$ be a subgroup of Aut $(\mathfrak{g}, \mathfrak{l})$ consisting of all the automorphisms $s$ such that $s(\mathfrak{h})=\mathfrak{h}, s \mid \mathfrak{l}^{\prime}$ is a diagram automorphism with respect to $\left(\mathfrak{l}^{\prime}, \mathfrak{l}^{\prime} \cap \mathfrak{h}, \Pi\right)$ and $s\left(Q_{\Sigma}\right)=Q_{\Sigma}$. Moreover we set $\operatorname{Aut}(\Delta, \Pi, \Sigma)=\left\{s \in G L\left(\mathfrak{h}^{*}\right): s(\Delta)=\Delta, s(\Pi)=\Pi, s(\Sigma)=\Sigma\right\}$. Then $G$ is isomorphic to Aut $(\Delta, \Pi, \Sigma)$ (cf. [Bou68, Ch.VIII, $\S 5$, no.1, Prop.5]). Thus we obtain the following proposition.

Proposition 41. Let $\mathfrak{G}=\left(\mathfrak{g},\left(\mathfrak{g}_{p}\right)_{p \in \mathbf{Z}}, \mathfrak{e}^{1}, \mathfrak{e}^{2}\right)$ be a pseudo-product $G L A$ of type $(\mathfrak{l}, M)$ such that $\operatorname{ad} \mathfrak{g}=\operatorname{Der}(\mathfrak{g})$. Then Aut $(\mathfrak{g})$ is a semi-direct product of $G$ by $\operatorname{Int}(\mathfrak{g})$. Furthermore $G$ is a isomorphic to $\operatorname{Aut}(\Delta, \Pi, \Sigma)$.

EXAMPLE. Let $\mathfrak{G}=\left(\mathfrak{g},\left(\mathfrak{g}_{p}\right)_{p \in \mathbf{Z}}, \mathfrak{e}^{1}, \mathfrak{e}^{2}\right)$ be of type $\left(A_{l}, \varepsilon_{1}, k \varpi_{l} ; A_{n}, \varpi_{1}\right)$. Then $\operatorname{Aut}(\Delta, \Pi, \Sigma)=\{1\}$, so Aut $(\mathfrak{g})=\operatorname{Int}(\mathfrak{g})$.

\section{References}

[ Bou58 ] N. Bourbaki, Algèbres, Ch.8, Hermann, Paris, 1958.

[Bou68] N. Bourbaki, Groupes et algèbres de Lie, Chaps 4,5,6, Hermann Paris, 1968.

[Bou75] N. Bourbaki, Groupes et algèbres de Lie, Chaps 7,8, Diffusion C.C.L.S., Paris, 1975.

[ Far88 ] R. Farnsteiner, Derivations and central extensions of finitely generated graded Lie algebras, J. Algebra, 118 (1988), 33-45.

[ Kac68 ] V.G. Kac, Simple irreducible graded Lie algebras of finite growth, Math. USSR-Izv., 32 (1968), 1271-1311.

[ Kac70] V.G. Kac, The classification of the simple Lie algebras over a field with nonzero characteristic, Math. USSR-Izv., 4 (1970), 391-413.

[ KN65 ] S. Kobayashi and T. Nagano, On filtered Lie algebras and geometric structure III, J. Math. Mech., 14 (1965), 679-706.

[Kos61] B. Kostant, Lie algebra cohomology and the generalized Borel-Weil theorem, Ann. of Math., 74 (1961), 329-387.

[Mor79] T. Morimoto, La géometrie des equations de Monge-Ampere, C.R. Acad. Sci. Paris, 289 (2 juillet 1979).

[Mor88] T. Morimoto, Transitive Lie algebras admitting differential systems, Hokkaido Math. J., 17 (1988), 45-88.

[ MT70] T. Morimoto and N. Tanaka, The classification of the real primitive infinite Lie algebras, J. Math. Kyoto Univ., 10-2 (1970), 207-243.

[ Tan70 ] N. Tanaka, On differential systems, graded Lie algebras and pseudo-groups, J. Math. Kyoto Univ., 10 (1970), 1-82.

[ Tan85] N. Tanaka, On affine symmetric spaces and the automorphism groups of product manifolds, Hokkaido Math. J., 3 (1985), 207-351.

[Yam82] K. Yamaguchi, Contact geometry of higher order, Japan J. Math., 8 (1982), 108-176.

[ Yat88 ] T. Yatsui, On pseudo-product graded Lie algebras, Hokkaido Math. J., 17 (1988), 
333-343.

[ Yat92 ] T. Yatsui, Simple graded Lie algebras of finite depth, Hokkaido Math. J., 21 (1992), $195-220$.

\section{Department of MATHEMATICS \\ HOKKAIDO UNIVERSITY}

SAPPORO 060, JAPAN 